\title{
Electron Microscopic Examination of Irradiated TRISO Coated Particles of Compact 6-3-2 of AGR-1 Experiment
}

The INL is a

U.S. Department of Energy

National Laboratory

operated by

Battelle Energy Alliance

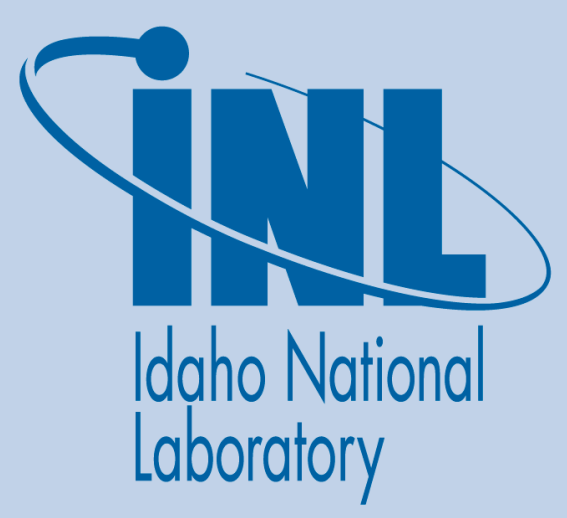

Isabella J van Rooyen

Brandon Miller

Dawn Janney

Jessica Riesterer

Paul Demkowicz

Jason Harp

Scott A Ploger

December 2012

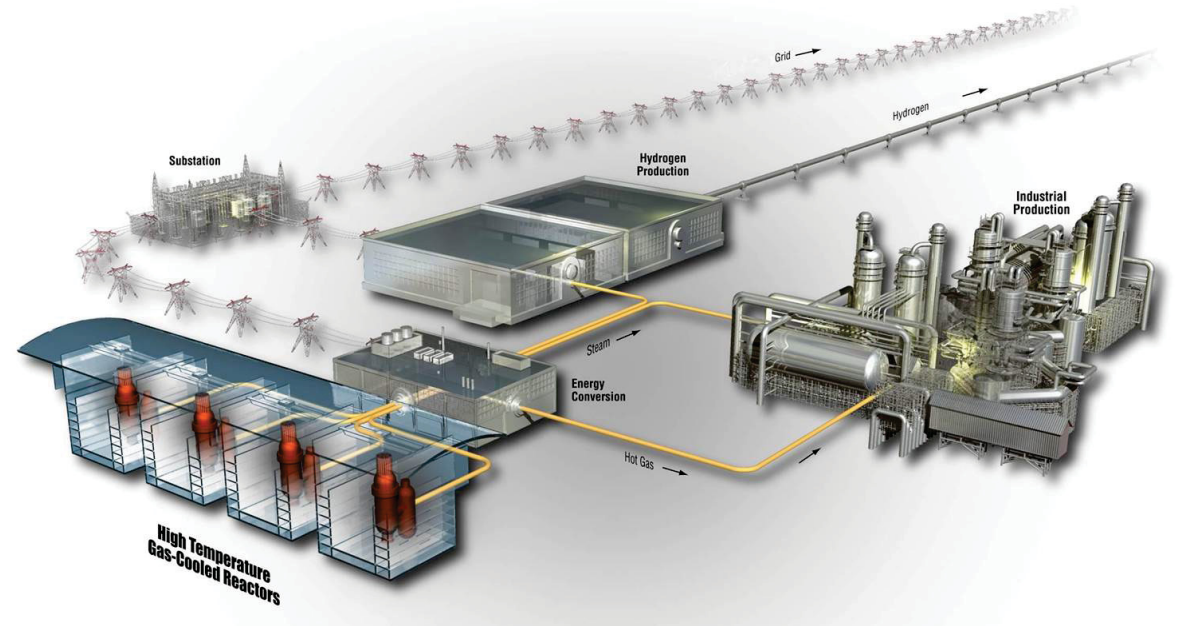




\section{DISCLAIMER}

This information was prepared as an account of work sponsored by an agency of the U.S. Government. Neither the U.S. Government nor any agency thereof, nor any of their employees, makes any warranty, expressed or implied, or assumes any legal liability or responsibility for the accuracy, completeness, or usefulness, of any information, apparatus, product, or process disclosed, or represents that its use would not infringe privately owned rights. References herein to any specific commercial product, process, or service by trade name, trade mark, manufacturer, or otherwise, does not necessarily constitute or imply its endorsement, recommendation, or favoring by the U.S. Government or any agency thereof. The views and opinions of authors expressed herein do not necessarily state or reflect those of the U.S. Government or any agency thereof. 


\title{
Electron Microscopic Examination of Irradiated TRISO Coated Particles of Compact 6-3-2 of AGR-1 Experiment
}

\author{
Isabella J van Rooyen \\ Brandon Miller \\ Dawn Janney \\ Jessica Riesterer \\ Paul Demkowicz \\ Jason Harp \\ Scott A Ploger
}

December 2012

\begin{abstract}
Idaho National Laboratory
VHTR Program
\end{abstract}

Fuel Performance and Design

Idaho Falls, Idaho 83415

http://www.inl.gov

Prepared for the

U.S. Department of Energy

Office of Nuclear Energy

Under DOE Idaho Operations Office

Contract DE-AC07-05ID14517 



\section{VHTR Program Fuel Performance and Design}

\section{Electron Microscopic Examination of Irradiated TRISO Coated Particles of Compact 6-3-2 of AGR-1 Experiment}

INL/EXT-11-23911

December 2012

Approved by:

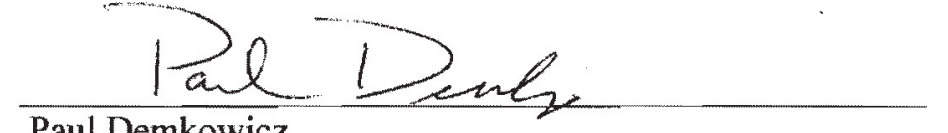

Paul Demkowicz

VHTR Fuels Post-irradiation Technical Lead

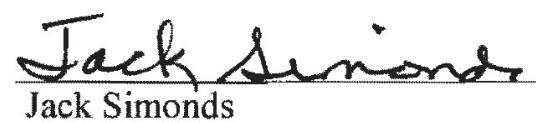

Fuel Development and Qualification Project Manager

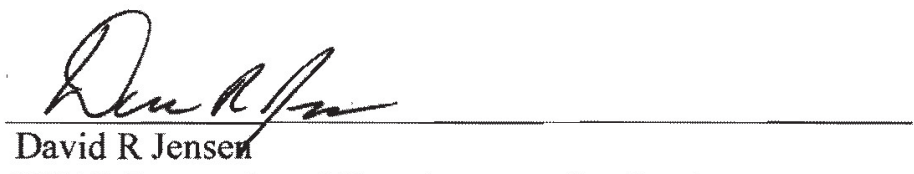

VHTR Research and Development Quality Assurance

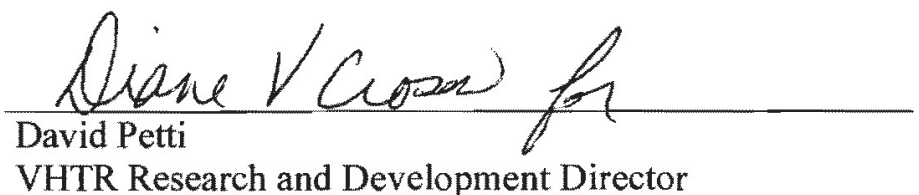

VHTR Research and Development Director
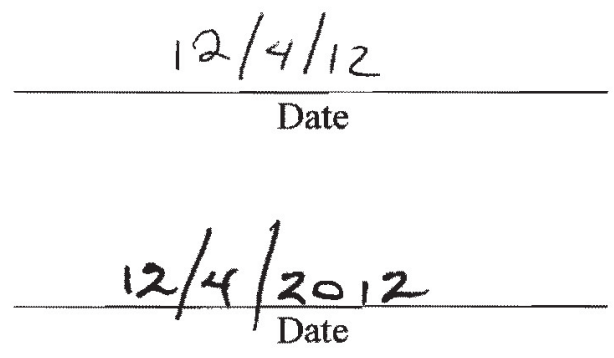

$12 / 4 / 12$ Date

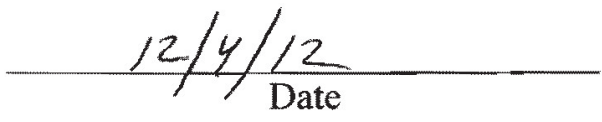




\section{ABSTRACT}

The electron microscopic examination of selected irradiated TRISO coated particles from AGR-1 fuel Compact 6-3-2 is presented in this report. Compact 6-3-2 refers to the compact in Capsule 6 at Level 3 of Stack 2. Capsule 6 contained AGR-1 "baseline" fuel as it is fabricated with refined coating process conditions designed to produce fuel with properties similar to those of historic German fuel, because of its excellent irradiation performance with $\mathrm{UO}_{2}$ kernels. The AGR-1 fuel is however made of low-enriched UCO (uranium oxycarbide). Kernel diameters are approximately $350 \mu \mathrm{m}$ with a U-235 enrichment of approximately $19.7 \%$. Compact 6-3-2 has been irradiated to $11.3 \%$ FIMA (fissions per initial heavy metal atom) compact average burnup with a time average, volume average temperature of $1070^{\circ} \mathrm{C}$ and with a compact average fast fluence of $2.38 \times 10^{21} \mathrm{n} / \mathrm{cm}^{2}$.

Four coated particles from Compact 6-3-2 were identified for electron microscopic examination based on irradiated microsphere gamma analysis results that indicated that they had Ag-110m inventory at the high end (CP34, CP35, CP39) and low end (CP30) of the distribution obtained from gamma counting 60 particles individually.

As this was the first irradiated AGR-1 compact examined using electron microscopy, a phased approached was followed to obtain information on fission product precipitate location and composition, and the effects of irradiation on the microstructure. The examination focused on the interface between the $\mathrm{SiC}$ (silicon carbide) to inner pyrolytic carbon (IPyC) layers. The detailed examination of $\mathrm{SiC}$ grain size and property parameters and the characterization of the fuel kernel will be presented in separate reports.

A new quantification approach on scanning electron microscopy (SEM) precipitate location was developed that yielded the following results for CP34:

- Clusters of Pd-rich precipitates were identified around the full circumference within and in close vicinity to the $\mathrm{SiC}-\mathrm{IPyC}$ interlayer up to an average $\mathrm{SiC}$ and IPyC depth of $6.7 \mu \mathrm{m}$ and $2.9 \mu \mathrm{m}$ respectively. It was found that the maximum depth of $\mathrm{Pd}$-rich precipitate $\mathrm{SiC}$ penetration was up to $\sim 15 \mu \mathrm{m}$, which corresponds with the transmission electron microscope (TEM) examination in one specific location.

- $\quad$ The SiC-IPyC interlayer was of an average thickness of $1.7 \mu \mathrm{m}$.

The Pd precipitates were found to be in the IPyC layer itself, in the SiC "fingers" and up to a maximum depth of approximately $15 \mu \mathrm{m}$ inside the $\mathrm{SiC}$ layer. These findings are different from those reported to date on the HFREU1bis experiments (11.5\% FIMA, $1250^{\circ} \mathrm{C}$ fuel sphere center temperature), where it was found that $\mathrm{Pd}$ is specifically present in the inner pyrocarbon $(\mathrm{PyC})$ layer at the inside of the $\mathrm{SiC}$ layer, but no Pd was mentioned to be found in the $\mathrm{SiC}$ layer itself.

All diffraction patterns from precipitates in the $\mathrm{SiC}$ layer in CP30, CP34, and $\mathrm{CP} 35$ could be produced by single crystals of $\mathrm{UPd}_{2} \mathrm{Si}_{2}$. Qualitative analyses of the energy-dispersive $\mathrm{x}$-ray spectroscopy (EDS) spectra showed significant 
concentrations of $\mathrm{U}, \mathrm{Pd}$, and $\mathrm{Si}$ with some spectra also having significant concentrations of $\mathrm{C}$ and $\mathrm{Zr}$. Semi-quantitative analyses of normalized atomic percentages of $\mathrm{Si}, \mathrm{Pd}$, and $\mathrm{U}$ showed similar compositions, with 40 at $\% \mathrm{Si}, 45$ at $\% \mathrm{Pd}$, and $10-15 \% \mathrm{U}$. This similarity in proportions of $\mathrm{U}, \mathrm{Pd}$, and Si suggest that these elements occurred in the same phase. Further investigation is also required to identify the phase containing $\mathrm{Zr}$, since $\mathrm{Zr}$ is present in significant concentrations in some precipitates but absent in spectra from other precipitates. This variation in the concentration of $\mathrm{Zr}$ relative to those of $\mathrm{U}, \mathrm{Pd}$, and $\mathrm{Si}$ suggests that the $\mathrm{Zr}$ is in a different phase, and that EDS spectra with $\mathrm{Zr}$ represent mixtures of $\mathrm{UPd}_{2} \mathrm{Si}_{2}$ with a $\mathrm{Zr}$-bearing phase. Even though $\mathrm{Zr}$ is a $4 \mathrm{~d}$ transition metal, its atomic radius is significantly different from that of $\mathrm{Pd}$, and an extensive $\mathrm{U}(\mathrm{Zr}, \mathrm{Pd})_{2} \mathrm{Si}_{2}$ solid solution seems unlikely. $\mathrm{U}$ and $\mathrm{Pu}$ often substitute for one another in metals and intermetallics, and it seems reasonable to speculate that low concentrations of $\mathrm{Pu}$ could substitute for $\mathrm{U}$ in $\mathrm{UPd}_{2} \mathrm{Si}_{2}$.

It is not possible to determine from the EDS data obtained from the L $\alpha$ x-rays whether low concentrations of Ag are present in the precipitates. . Since Ag and Pd have identical atomic radii (both $0.144 \mathrm{~nm}$ for the pure material), it seems reasonable to speculate that any $\mathrm{Ag}$ atoms that might be present would substitute for $\mathrm{Pd}$ atoms in a $\mathrm{U}(\mathrm{Ag}, \mathrm{Pd})_{2} \mathrm{Si}_{2}$ solid solution rather than forming a separate phase.

Cesium was also identified in the presence of Pd in CP35 in some areas in contrast to the areas evaluated for CP34 and CP30. It appears that the Cscontaining precipitates are not located only on the grain boundaries, but within individual grains; however, this needs to be confirmed with further analysis of the data. It is further noted that indications of $\mathrm{Zr}$ have also been found along with the Cs-containing precipitates.

No evidence was found of any phase transformation of the $\mathrm{SiC}$ from the cubic to the hexagonal phase because of the irradiation cycle as determined by selected area diffraction patterns measured on the different TEM samples prepared from $\mathrm{CP} 34$ and $\mathrm{CP} 35$. Although possibly one hexagonal structure for $\mathrm{CP} 30$ was observed it is not possible to state that this hexagonal phase is because of the irradiation cycle, as it is known from previous studies that small amounts of hexagonal $\mathrm{SiC}$ may exist in chemical vapor deposited 3C-SiC because of the manufacturing conditions. It is thus recommended that conclusions on this finding be reserved until more statistical data are available on unirradiated AGR-1 fuel. It is further expected that the electron backscatter diffraction analysis of these layers will provide more statistical data on the presence of hexagonal phases because of the irradiation.

Intensity variations in the IPyC diffraction ring patterns after irradiation are observed for CP34 and CP35 and are an indication that the PyC is becoming anisotropic. Intensity variations within CP34 are not significant, although a difference is observed between those for CP34 and CP35 and those of unirradiated material. At this point it cannot be determined if temperature or irradiation damage is the predominant reason for the observed changes. More work is in needed to compare these diffraction patterns using the same aperture to eliminate aperture contributions.

Although a full investigation on the loop and dislocation densities of this material was not completed, no significant difference between the inner and outer 
portions of SiC layer for CP34 was observed in the specific area of examination. However, differences in loop and dislocation densities were observed at different locations along the circumference of the SiC layer. No denuded zone for dislocation loops along grain boundaries were observed during this study, but previous studies typically found denuded zones at grain boundaries in some areas for irradiated $\mathrm{SiC}$ at $1130^{\circ} \mathrm{C}$. This may be of importance with respect to fission product transport mechanisms and will be investigated specifically in the areas where precipitates have been found. At this point, no conclusion has been reached from this observation and additional analysis is needed. The cavities (or voids) due to irradiation damage, were predominantly found to be spherical in shape for CP34 in contrast with angular shaped cavities found in CP35. Other researchers have typically found that the shape of cavities changes from spherical to facetted as a function of irradiation temperature. Both the findings on the IPyC diffraction patterns and the cavity shape differences suggest that CP35 may have been exposed to higher temperatures than that of CP34. No debonding or macroscopic cracks were observed in the SiC-IPyC interlayer in all the samples from CP34, CP35, and CP30 examined in this study.

Two preliminary differences between the microstructure of the CP30 (high Ag-110m release) and those of CP34 and CP35 (low Ag-110m release) were observed, but the significance of these observations could not be determined at this stage, as these observations need to be validated with results obtained from future compacts. The two differences observed are as follows:

- No cavities due to irradiation were observed in the SiC grains during TEM examination of $\mathrm{CP} 30$, whereas cavities were observed in the microstructure of the $\mathrm{SiC}$ grains of both $\mathrm{CP} 34$ and CP35. This observation may suggest that CP30 was exposed to lower temperatures than CP34 and CP35.

- The EDS analysis of CP30 showed that the coating layers did not contain detectable concentrations of $\mathrm{Pu}$ in contrast with those found in CP34 and CP35.

Future plans include the verification of precipitate composition using TEM EDS with $\mathrm{K} \alpha \mathrm{x}$-rays to differentiate between $\mathrm{Ag}, \mathrm{Pd}$ and $\mathrm{U}$. It is also recommended that a detailed precipitate analysis be done using an electron probe microanalyzer and LEAP techniques, in conjunction with more in-depth investigation of the fission product presence in inter and transgranular locations in the $\mathrm{SiC}$ grains. More detailed and quantitative studies on cavities and dislocation loops will be pursued and changes in PyC anisotropy caused by irradiation and temperature will be investigated. 


\section{ACKNOWLEDGEMENT}

Acknowledgement is given to Jim Madden for contribution towards the focused ion beam sample preparation of the transmission electron microscope samples. 


\section{CONTENTS}

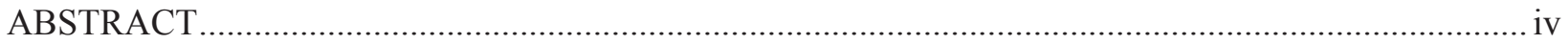

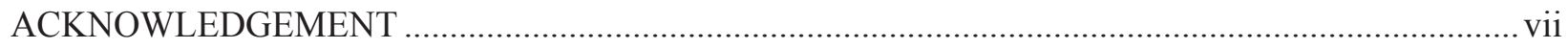

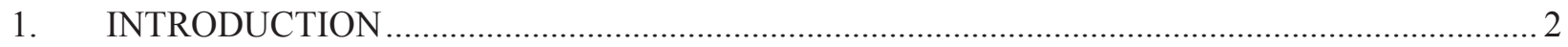

2. OBJECTIVES OF ELECTRON MICROSCOPIC EXAMINATION ….......................................... 4

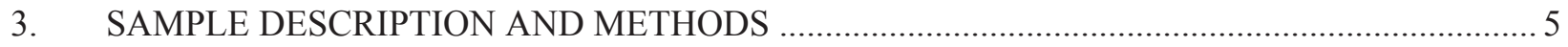

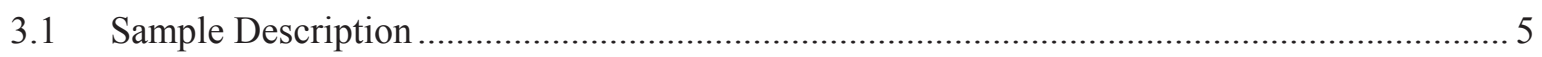

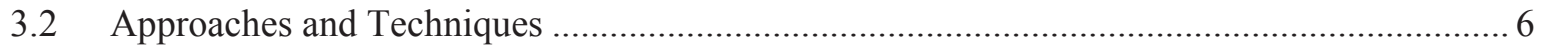

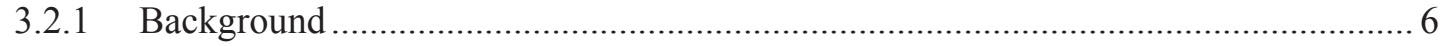

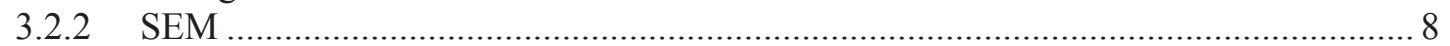

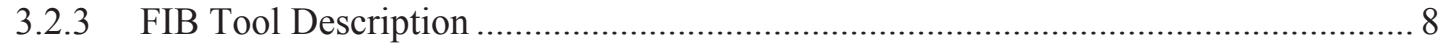

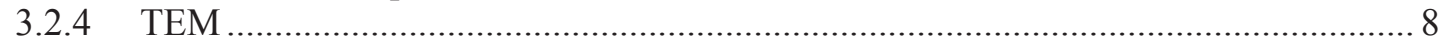

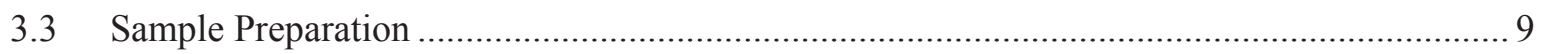

3.3.1 Particle Mounting, Grinding, and Polishing ….................................................... 9

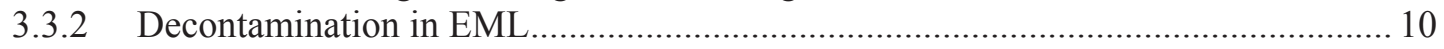

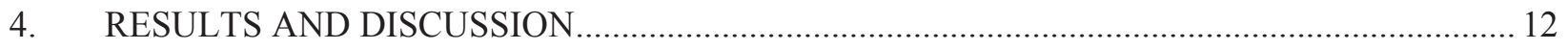

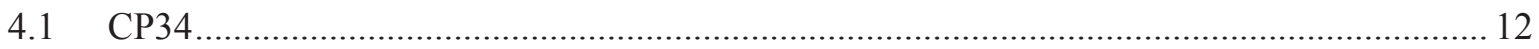

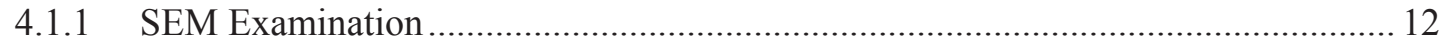

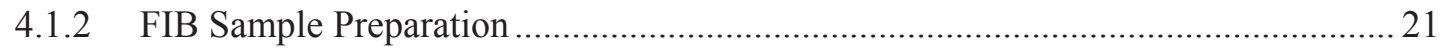

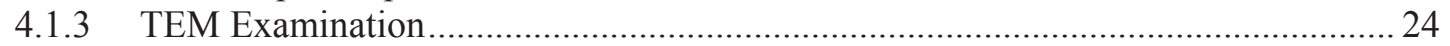

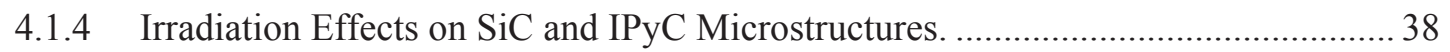

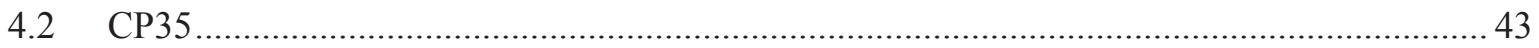

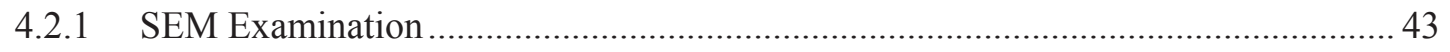

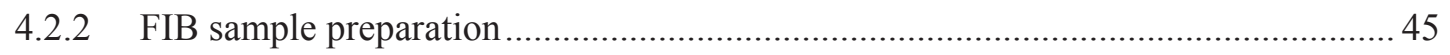

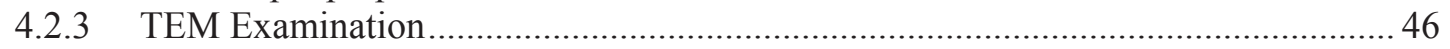

4.2.4 Irradiation Effects on $\mathrm{SiC}$ and PyC Microstructures............................................... 48

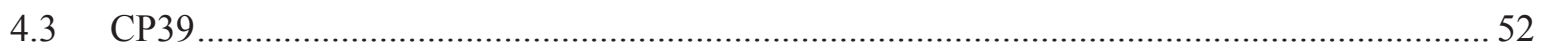

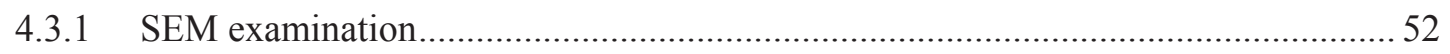

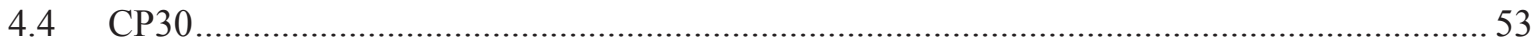

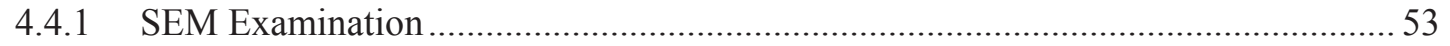

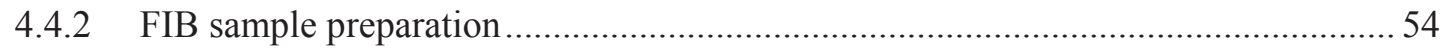

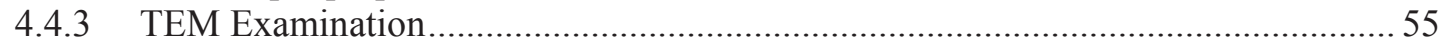

4.4.4 Irradiation Effects on $\mathrm{SiC}$ and PyC Microstructures............................................... 56

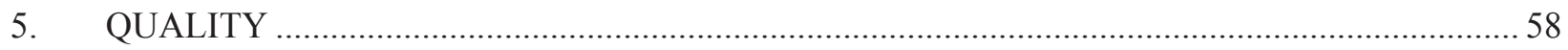

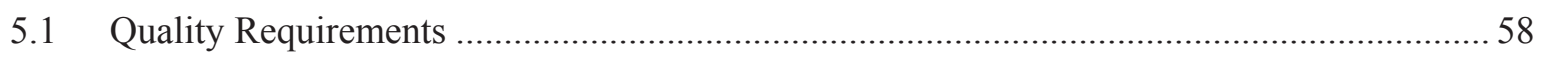

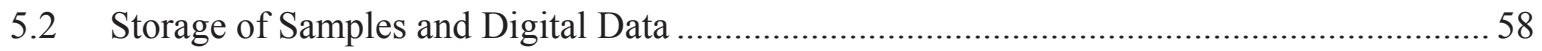

5.3 Skill of Performers (Electron Microscopists) and Principal Investigator ............................ 58

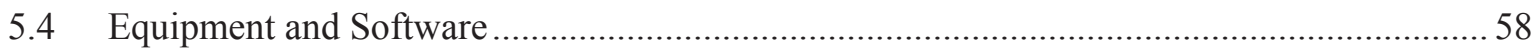

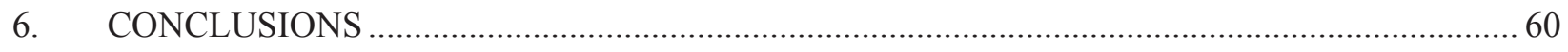

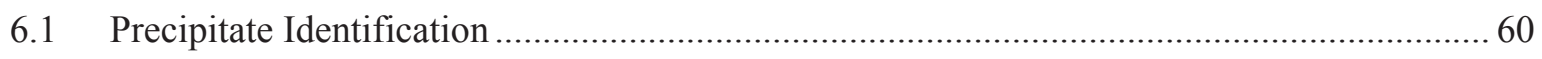




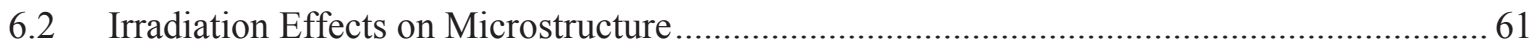

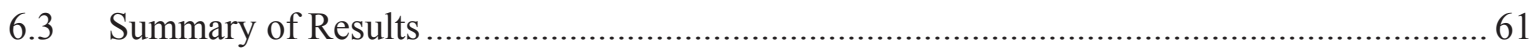

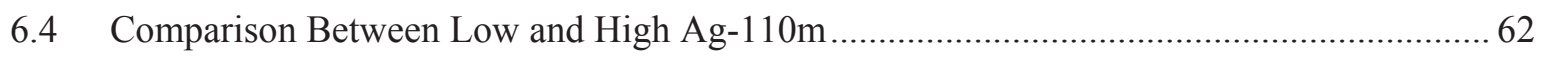

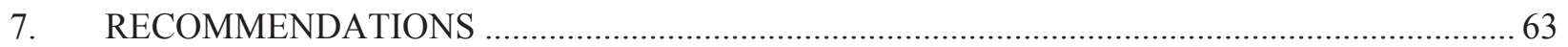

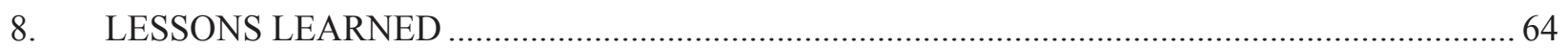

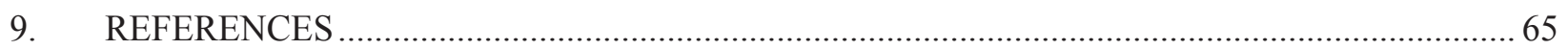

\section{FIGURES}

Figure 1. Numbering scheme for AGR-1 Compact 6-3-2 ...................................................................2

Figure 2. Normalized activity ratio distribution of 60 particles from Compact 6-3-2. Location of particles $30,34,35$, and 39 within the distribution are indicated in the figure.......................... 5

Figure 3. SEM examination plan for CPs from Compact 6-3-2. .......................................................... 6

Figure 4. TEM examination plan for CP34 and CP35 from Compact 6-3-2, based on SEM

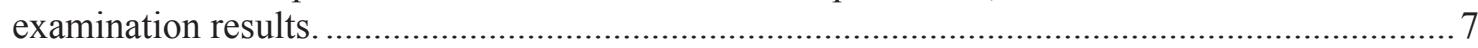

Figure 5. Diagram indicating the state of the met mount of CP30 from Compact 6-3-2 …................... 10

Figure 6. Interlayer thickness measurement schematic presentation. .................................................... 13

Figure 7. IPyC-SiC interlayer thickness measured from SEM montage (zero values in locations where FIB sample preparation was completed) ................................................................. 13

Figure 8. Schematic presentation to show measurement of furthest precipitate from Interlayer............... 14

Figure 9. Precipitate position as a function of the distance away from the IPyC-SiC interlayer............... 15

Figure 10. Number of precipitates located in the SiC and IPyC layers of CP34 based on SEM montage.

Figure 11. CP34 SEM montage showing randomly distributed precipitate clusters encircled by blue.

Figure 12. Pd rich precipitates from two different locations showing precipitates located both on the $\mathrm{SiC}$-fingers as well as in the PyC near the IPyC-SiC interlayer self.

Figure 13. SEM and EDS analysis of CP 34 of Compact 6-3-2, revealing the Pd-containing precipitates in both the $\mathrm{SiC}$ and IPyC layers.

Figure 14. SEM, EDS and WDS analysis of CP 34 of Compact 6-3-2, revealing the cluster of Pd precipitates on the $\mathrm{SiC}-\mathrm{IPyC}$ interface as well as more discrete $\mathrm{Pd}$ precipitates in the $\mathrm{SiC}$ layer itself (these Pd precipitates may also contain $\mathrm{U}$ ).

Figure 15. Typical expanded section of EDS spectrum showing details of overlaps between Ag, $\mathrm{Pd}$, and $\mathrm{U}$ (EDS spectrum taken from CP30 picture 13 in original EML database). See Table 2 for energies for Ag X-rays.

Figure 16. Secondary electron image showing the edge effect because of polishing resulting in an approximately $2 \mu \mathrm{m}$ region of significant relief in the $\mathrm{SiC}$ layer.

Figure 17. FIB sample preparation position for CP34 from Compact 6-3-2. .........................................22

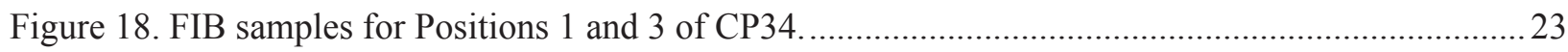


Figure 19. FIB samples prepared at Position 2 of CP34 showing location of sample as well as precipitates clearly visible in the $\mathrm{SiC}$ matrix.

Figure 20. TEM micrograph showing representative images of precipitates in the IPyC layer of CP34 from Compact 6-3-2, TEM Position 1a.

Figure 21. TEM micrograph showing a precipitate with nanometer-sized grains in CP34 from Compact 6-3-2, TEM Position 1b.

Figure 22. TEM micrographs for Specimens $2 \mathrm{a}, 2 \mathrm{~b}$, and $2 \mathrm{c}$ showing that precipitates are visible in Position $2 \mathrm{a}$ and $2 \mathrm{~b}$, but no precipitates were observed at Position 2c.

Figure 23. TEM micrograph showing representative images of precipitates in the $\mathrm{SiC}$ layer of CP34 from Compact 6-3-2, TEM Position 2b.

Figure 24. TEM micrograph showing selected images of precipitates on the $\mathrm{SiC}$ grain boundaries as well as in the IPyC of CP34 from Compact 6-3-2, TEM Position 3.

Figure 25. Zone-axis diffraction patterns from precipitates in CP34, indexed as $\mathrm{UPd}_{2} \mathrm{Si}_{2}$ showing a) zone [010] from CP 34 sample $2 b$ picture 5, b) zone [110] from CP34 sample 2b, c) zone [111] from CP34 Sample 2a.

Figure 26. Three additional zone-axis diffraction patterns indexed as $\mathrm{UPd}_{2} \mathrm{Si}_{2}$ showing a) zone [2 $-4-1$ ] from the same precipitate as part a) in Figure 29, with arrow showing a discontinuous ring that is not part of the single-crystal pattern. b) zone [ $1-20]$. c) zone [2 4 -1], considering bright reflections only.

Figure 27. A precipitate surrounded by $\mathrm{SiC}$; fine-scale contrast variations similar to those shown here are commonly observed in precipitates in AGR-1 SiCand IPyC matrices.

Figure 28. EDS spectrum showing small peak at $\sim 14.2 \mathrm{keV}$ that could be $\mathrm{Pu}$ or $\mathrm{Sr}$ and small peak at $\sim 18.3 \mathrm{keV}$ that could be $\mathrm{Pu}$ or $\mathrm{U}$ (original EML database identification: CP34 Sample 3, Picture 7, Spot A). .36

Figure 29. Expanded section of EDS spectrum showing details of overlaps between the Sr Kseries peaks, $\mathrm{Pu}$ L-series peaks, and peaks from $\mathrm{U}$ and $\mathrm{Zr}$ (Original EML database identification: CP34 sample 2B Picture 2, Spot B). See Table 4 for Pu and Sr X-ray energies.

Figure 30. Expanded section of an EDS spectrum showing only peaks from U. Note that there is no U L $39-M 5$ peak, even though the intensity in this spectrum is greater than that in Figure 29. (Original EML database identification: CP30 Picture 13).

Figure 31. Summary of representative selected area diffraction patterns of the SiC layer of CP34 determined at different positions.

Figure 32. Bright Field TEM images showing irradiated microstructure of CP34 Position 2a, with (a) and (b) showing the typical dark spot defects and (c) showing the cavities caused by irradiation.

Figure 33. Bright field TEM images of the irradiated SiC microstructure of CP34 showing (a) and (b) variations in loop density at Positions $2 \mathrm{c}$ and (c) at Position 3, and (d) shows spherical cavities at Position 3.

Figure 34: Image of unirradiated IPyC showing randomly oriented crystals 5-10 nm across with corresponding SAD pattern. .41

Figure 35. Bright field TEM image showing the typical IPyC microstructure of CP34. .42 
Figure 36. Selected area diffraction patterns as examples of preliminary data on IPyC structure of (a) CP34 Position 1a, (b) CP34 Position 1b, and (c) CP34 Position 3 (aperture sizes were not recorded during time of analysis and may not be the same for all the diffraction patterns).

Figure 37. Bright field TEM image shows no evidence of SiC-IPyC interface debonding due to irradiation in CP34...

Figure 38. Secondary electron images of CP 35 from Compact 6-3-2.

Figure 39. SEM micrographs of CP35 of Compact 6-3-2 showing precipitates (Pd-rich) in the buffer, IPyC layer and at the SiC-IPyC interlayer.

Figure 40. FIB sample preparation detail of CP35 from Compact 6-3-2.

Figure 41. TEM micrograph showing Pd- U precipitates in the SiC layer in FIB Position 6 for CP35 from Compact 6-3-2.

Figure 42. BF TEM micrograph showing Cs precipitates in the wormlike structure and SiC grain (Note: Ag is not confirmed due to peak overlap).

Figure 43. BF TEM micrograph with EDS spectra showing apparent Cs-containing precipitates in individual $\mathrm{SiC}$ grains of $\mathrm{CP} 35$ at Position 6.

Figure 44. Summary of representative selected area diffraction patterns of the SiC layer of CP35 determined at different positions.

Figure 45. Bright field TEM images of the irradiated SiC microstructure of CP35 showing (a) typical loops because of irradiation, (b) higher magnification shows the collection of loops at the stacking faults and (c) showing that the cavities are angular shaped.

Figure 46. Selected area diffraction pattern as preliminary data on IPyC structure of CP34 Position 6. (aperture size was not recorded during time of analysis and may not be the same for all the diffraction patterns)......

Figure 47. BF TEM image of the SiC-IPyC of CP35 shows no evidence of debonding.

Figure 48. Back scattered electron images of CP 39 of Compact 6-3-2, showing surface contamination. Inset at upper right is an optical micrograph.

Figure 49. FIB sample preparation position for CP30 from Compact 6-3-2. .54

Figure 50. TEM micrograph showing representative images of precipitates in the $\mathrm{SiC}$ layer of particle 30 from Compact 6-3-2, TEM position 1. Bright field TEM images of the irradiated $\mathrm{SiC}$ microstructure of CP30 showing (left) typical black spots (representative of dislocation loops) because of irradiation and (right) no debonding of the SiC-PyC interlayer. .55

Figure 51. Diffraction pattern indexed as the [010] zone axis of $\mathrm{UPd}_{2} \mathrm{Si}_{2}$. .56

Figure 52. Summary of representative selected area diffraction patterns of the SiC layer of CP30 determined at different positions.

\section{TABLES}

Table 1. Table describing the electron microscopic techniques used to achieve objectives...

Table 2. Relevant x-rays for qualitatively identifying low concentrations of Ag in spectra from areas with much higher concentrations of Pd and U. 
Table 3. TEM-EDS analyses from CP34, normalized so that the sum of the elements shown is $100 \%$

Table 4. Relevant x-rays for distinguishing between low concentrations of $\mathrm{Sr}$ and $\mathrm{Pu}$, showing peak overlaps in spectra with much higher concentrations of $\mathrm{Si}, \mathrm{U}, \mathrm{Pd}$, and possibly some $\mathrm{Zr}^{4,5}$...

Table 5. Study results.. 


\section{ACRONYMS}

AGR Advanced Gas Reactor

ATR Advanced Test Reactor

BF Bright Field

EDS Energy-dispersive x-ray spectroscopy

CP coated particle

EML Electron Microscopy Laboratory

EPMA electron probe microanalyzer

FIB focused ion beam

FIMA fissions per initial metal atom

HFEF Hot Fuel Examination Facility

ICP-MS inductively coupled plasma-mass spectrometry

ID identification

IMGA irradiated microsphere gamma analysis

INL Idaho National Laboratory

IPyC inner pyrolytic carbon

LBL leach-burn-leach

LEAP Local Electrode Atom Probe

NGNP Next Generation Nuclear Plant

OPyC outer pyrolytic carbon

ORNL Oak Ridge National Laboratory

PIE post-irradiation examination

PyC pyrocarbon

SEM scanning electron microscope

TEM transmission electron microscope

TRISO tristructural isotropic

VHTR very high temperature reactor

WDS wavelength-dispersive x-ray spectroscopy 


\section{Electron Microscopic Examination of Irradiated TRISO Coated Particles of Compact 6-3-2 of AGR-1 Experiment}

\section{INTRODUCTION}

This document presents results obtained from the electron microscopic examination of selected irradiated tristructural isotropic (TRISO) coated particles (CPs) taken from fuel Compact 6-3-2 irradiated in Advanced Gas Reactor (AGR) experiment AGR-1. Background is given to provide the context of these CPs in the larger context of the Next Generation Nuclear Plant (NGNP) Fuel Development and Qualification Program. The overarching goal of the NGNP Fuel Development and Qualification Program program is to provide a baseline fuel qualification data set to support licensing and operation of a very high temperature reactor (VHTR). To achieve these goals, the program includes the elements of fuel fabrication, irradiation, post-irradiation examination (PIE) and accident testing, fuel performance, and fission product transport. ${ }^{1}$

A series of fuel irradiation experiments are planned in the Advanced Test Reactor (ATR) at Idaho National Laboratory (INL). AGR-1, the first of these irradiation experiments, began in the ATR in December of 2006 and ended in November 2009. The PIE activities, which include electron microscopy of irradiated particles as described in PLN-2828, ${ }^{2}$ began in March 2010. This report presents the initial results of the electron microscopic examination of Compact 6-3-2 from Capsule 6, which began in June 2011.

This unique identification number 6-3-2 is based on the specific capsule, level, and stack number for each compact. Figure 1 identifies the stack and position (or level) numbers in a particular capsule. Compact 6-3-2 refers to the compact in Capsule 6 at Level 3 of Stack 2.

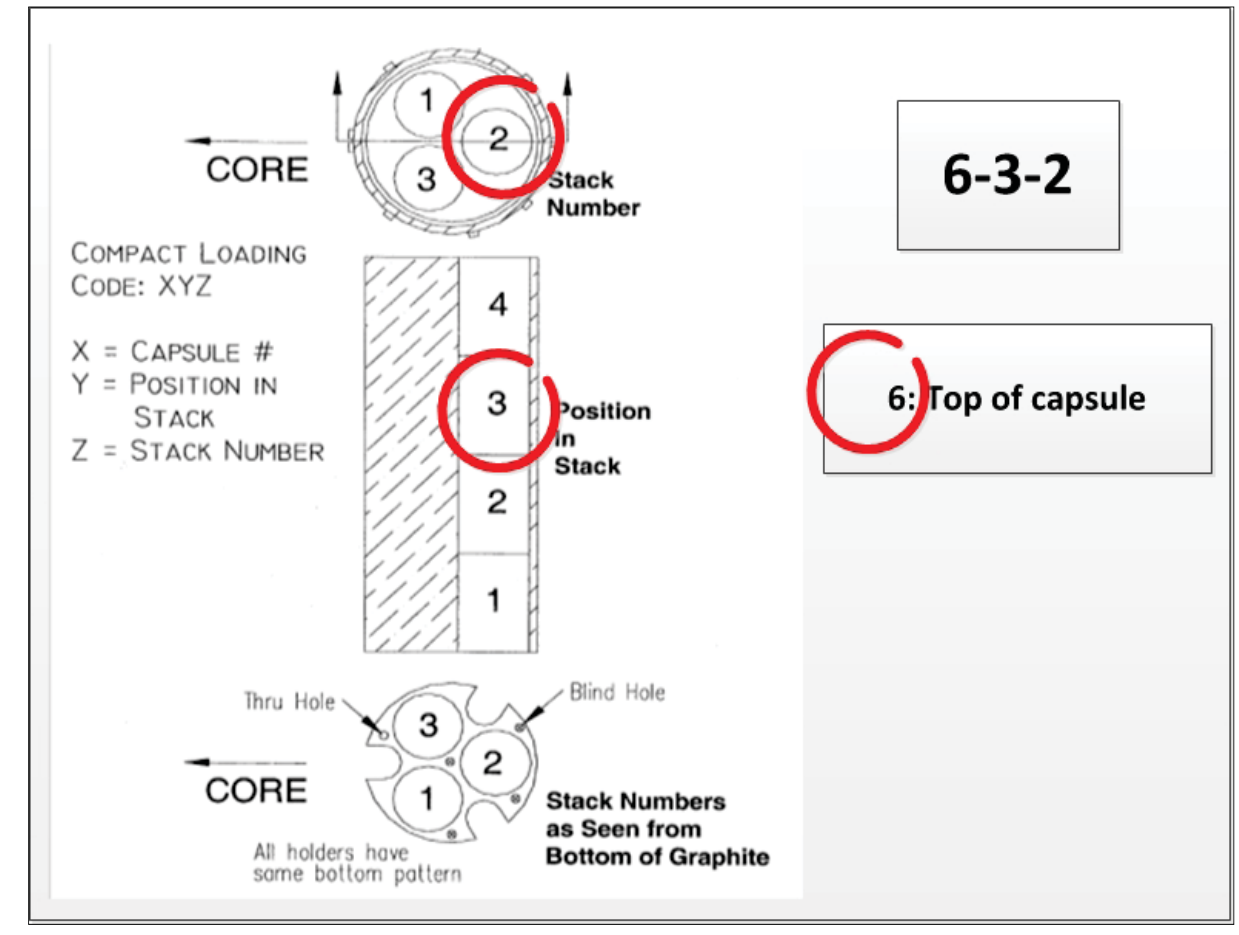

Figure 1. Numbering scheme for AGR-1 Compact 6-3-2. 
Capsule 6 contains baseline fuel fabricated to have properties similar to those of historic German fuel because of its excellent irradiation performance with $\mathrm{UO}_{2}$ kernels. The AGR-1 fuel is, however, made of low-enriched UCO (uranium oxycarbide). Kernel diameters are approximately $350 \mu \mathrm{m}$ with a U-235 enrichment of approximately $19.7 \%$.

Along with the baseline fuel, the AGR-1 irradiation experiment also includes three different fuel variants. The electron microscopy results of these variants will be presented in separate reports. Each of the fuel variants represents a particular deviation in the processing parameters of either the inner pyrolytic carbide (IPyC) or SiC (silicon carbide) coating layers compared to the baseline fuel as follows:

- Baseline. Because of its excellent irradiation performance with $\mathrm{UO}_{2}$ kernels, coating process conditions used to fabricate historic German fuel were chosen as the starting point for the baseline fuel. Parametric studies refined these conditions for the specific coater to be used to coat AGR-1 fuel.

- Variant 1. The IPyC coating temperature was increased relative to the baseline process (from 1265 to $1290^{\circ} \mathrm{C}$ ) for this variant. This change is expected to enhance the irradiation dimensional stability of the pyrocarbon $(\mathrm{PyC})$, but with increased permeability and resulting uranium dispersion. Also, the IPyC layer density is slightly lower than the baseline density.

- Variant 2. The IPyC coating gas fraction was increased relative to the baseline process (from 0.3 to 0.45 ) for this variant. This change is also expected to enhance the irradiation dimensional stability of the PyC but without significantly increasing uranium dispersion. Also, the IPyC layer density is slightly higher than the baseline density.

- Variant 3. The carrier gas composition for the SiC layer deposition was changed from $100 \%$ hydrogen to a $50 \%$ argon-50\% hydrogen mixture and the deposition temperature was lowered relative to the baseline (from 1500 to $1425^{\circ} \mathrm{C}$ ). This change is expected to reduce the potential for $\mathrm{SiC}$ defects resulting from uranium dispersion and provide a variation in $\mathrm{SiC}$ microstructure that may be less permeable to metallic fission products. 


\section{OBJECTIVES OF ELECTRON MICROSCOPIC EXAMINATION}

The main objective of the electron microscopic examination of fuel particles is to characterize microstructure to establish irradiation effects, fuel kernel porosity, layer degradation or corrosion, fission product precipitation, layer debonding, and grain size and boundary property determination. Migration of fission products from the kernels across the TRISO layers will also be examined. In particular, corrosion of the $\mathrm{SiC}$ layers by fission products such as palladium, resulting in localized layer thinning, will be investigated. Another objective is to determine if there are microstructure differences between particles that exhibited high and low releases of Ag-110m. The interpretation and discussion of results presented in this report focus on the IPyC and $\mathrm{SiC}$ layers with only brief reporting on fuel kernel observations. The detailed technical discussion of the fuel kernels will be presented in a separate report at a later stage. The detailed grain size and grain boundary properties of the irradiated samples will be reported later. 


\section{SAMPLE DESCRIPTION AND METHODS}

\subsection{Sample Description}

Compact 6-3-2 was irradiated to $11.3 \%$ fissions per initial metal atom (FIMA) compact average burnup with a time-average, volume-average temperature of $1070.2^{\circ} \mathrm{C}$ and with a compact average fast fluence of $2.38 \times 10^{21} \mathrm{n} / \mathrm{cm}^{2}$. Four CPs of Compact 6-3-2 were identified for electron microscopic examination based on irradiated microsphere gamma analysis (IMGA) results that indicated they had Ag-110m inventory at the high end (CP34, CP35, CP39) and low end (CP30) of the distribution obtained from gamma counting 60 particles individually. The ratio of measured Ag-110m to Cs-137 activity was used to minimize the effects of variations in fissile content of the kernel (due to differences in size, stoichiometry, or density). Figure 2 shows a distribution of the normalized Ag-110m/Cs-137 activity ratios for Compact 6-3-2 particles, indicating the location in the distribution for each of the four particles examined in this study. Note that particles with lower ratios (left side of the distribution) apparently released a greater fraction of their silver inventory during irradiation compared to particles with higher ratios. Data denoted by red columns in Figure 2 represents particles that had no detectable Ag- $110 \mathrm{~m}$ activity; the detection limit was instead used as a conservative high activity value to calculate the normalized ratio. All four of the particles examined in this study had detectable Ag-110m activity.

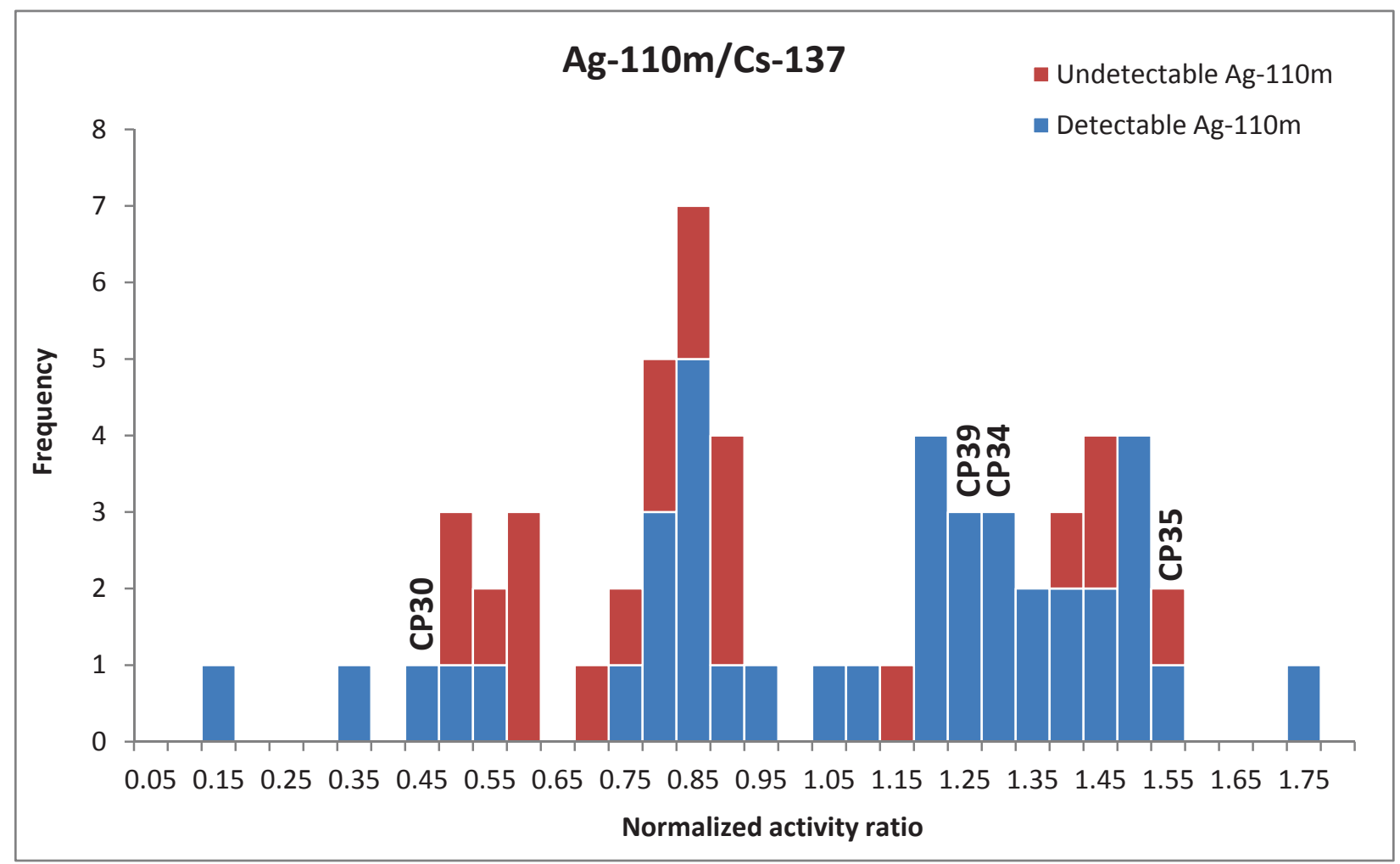

Figure 2. Normalized activity ratio distribution of 60 particles from Compact 6-3-2. Location of particles 30, 34,35 , and 39 within the distribution are indicated in the figure. 


\subsection{Approaches and Techniques}

\subsubsection{Background}

A phased approach was used to achieve the objectives stated in Section 2. Initial screening and basic examination with a scanning electron microscope (SEM) identified specific areas or features of interest. A plan for SEM examination typically includes identifying fission product precipitation, SiC attack, SiC grain sizes, and fuel kernel features as shown in Figure 3. A transmission electron microscope (TEM) investigation plan was then prepared based on the knowledge gained from the basic SEM examination as shown in Figure 4. The TEM plan first identified the locations where samples for TEM examination would be prepared using the focused ion beam (FIB), followed by more detailed studies during the TEM investigation. In Figure 4, the orange lines correspond to the approximate locations where thin TEM lamella were extracted from the cross-sectioned particles. Note that the TEM examinations focused on the interface regions between coating layers, in particular the IPyC-SiC interface.

Other advanced microscopic techniques, for example Local Electrode Atom Probe (LEAP), electron energy-loss spectroscopy (EELS) and high resolution transmission electron microscopy (HRTEM) are not included in this examination at present mainly because of challenges in sample preparation and transport of irradiated samples from one facility to another. Table 1 summarizes the techniques used to reach the electron microscopic examination objectives.

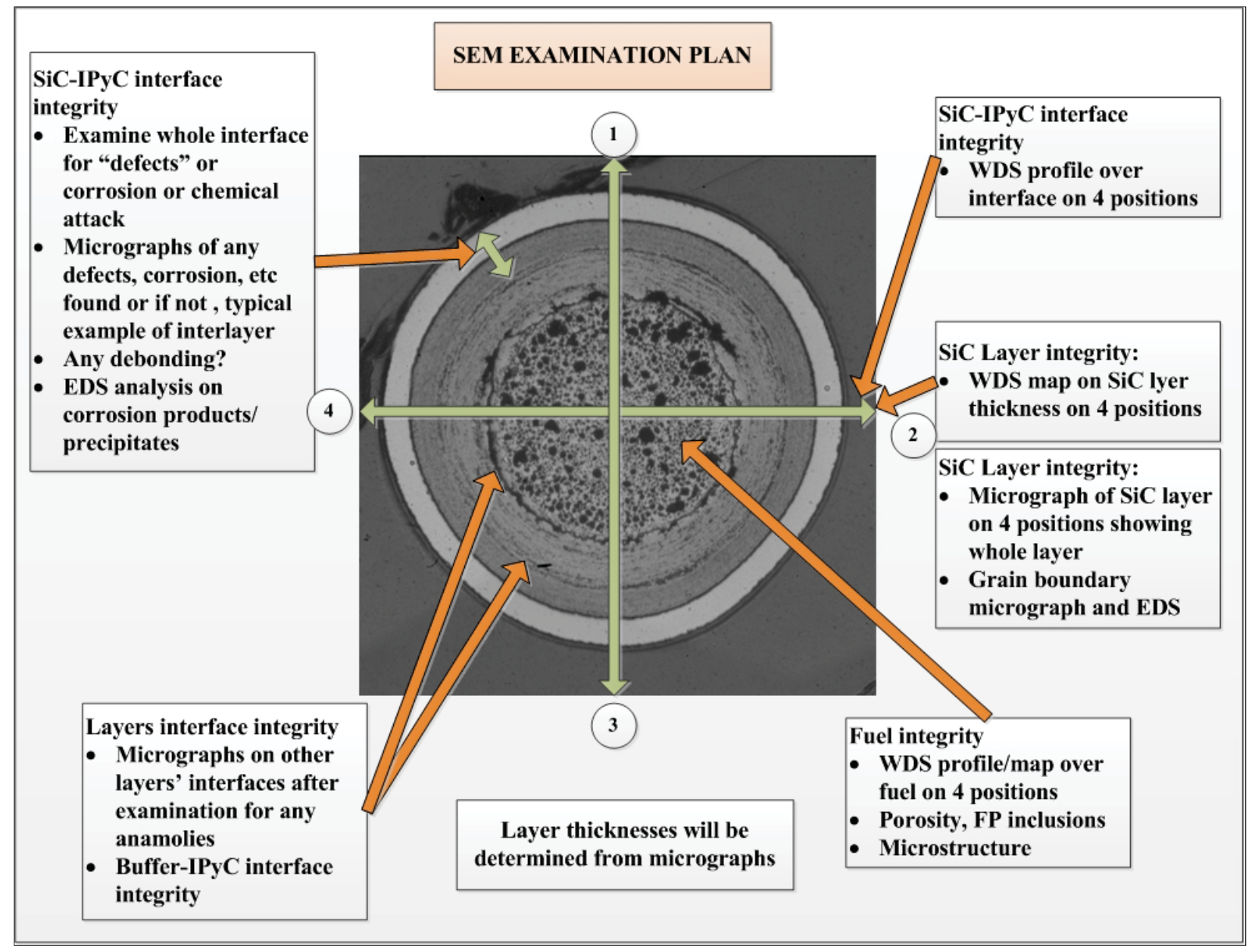

Figure 3. SEM examination plan for CPs from Compact 6-3-2. 


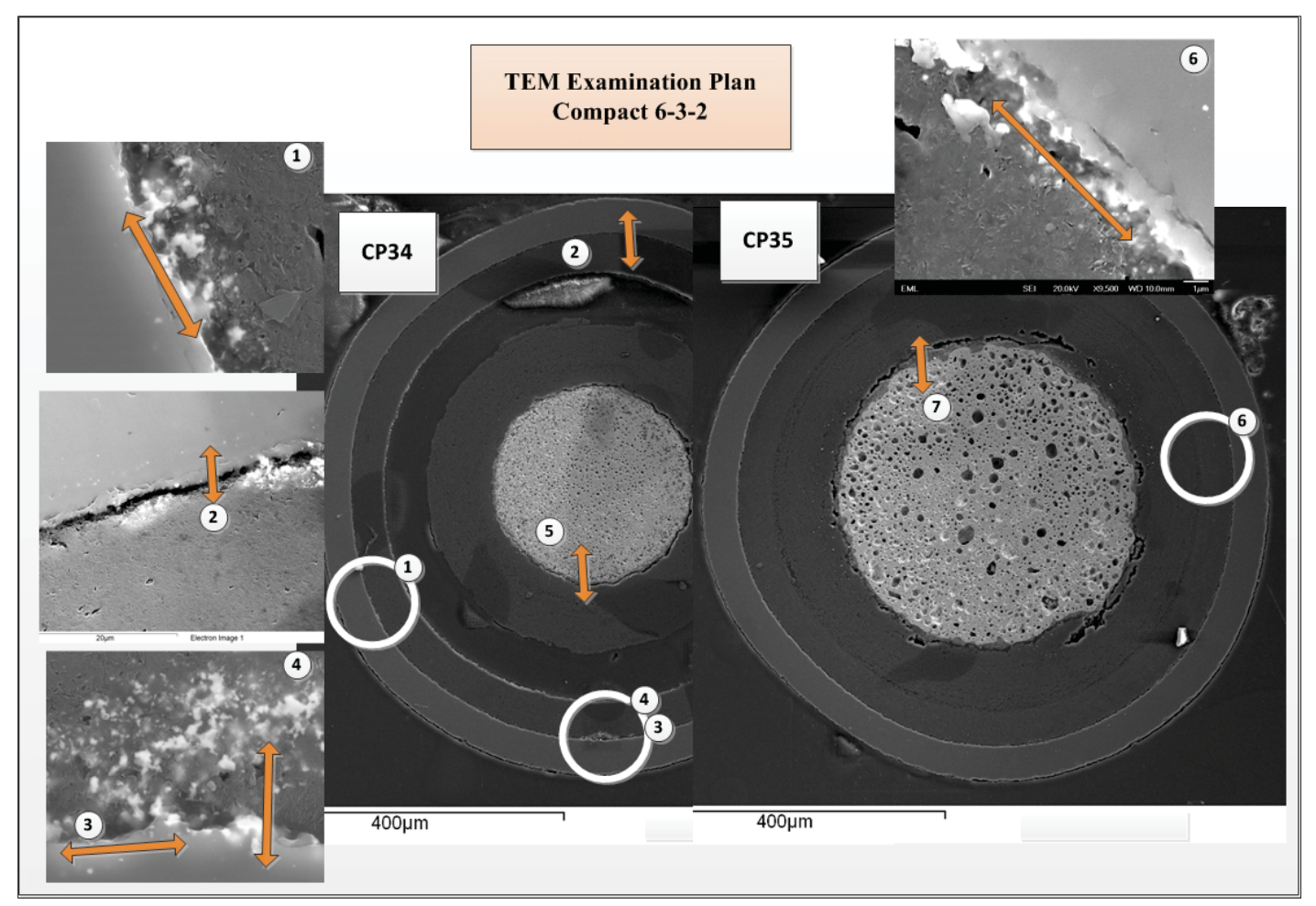

Figure 4. TEM examination plan for CP34 and CP35 from Compact 6-3-2, based on SEM examination results.

Table 1. Table describing the electron microscopic techniques used to achieve objectives.

\begin{tabular}{|c|c|}
\hline Objective & $\begin{array}{l}\text { Electron microscopic technique: } \\
\text { Basic examination (Phase 1) }\end{array}$ \\
\hline $\begin{array}{l}\text { Microstructure to } \\
\text { establish irradiation } \\
\text { effects }\end{array}$ & $\mathrm{N} / \mathrm{A}$ \\
\hline $\begin{array}{l}\text { Layer degradation or } \\
\text { corrosion }\end{array}$ & $\begin{array}{l}\text { SEM: Basic examination to identify the } \\
\text { location of any corrosion or thinning } \\
\text { (oxidization) }\end{array}$ \\
\hline \multirow{2}{*}{$\begin{array}{l}\text { Fission product } \\
\text { precipitation and } \\
\text { identification }\end{array}$} & $\begin{array}{l}\text { SEM: Basic examination to identify the } \\
\text { location of fission product precipitates }\end{array}$ \\
\hline & $\begin{array}{l}\text { SEM-EDS and SEM-WDS: Detection } \\
\text { thresholds are typically at the level of } \\
\text { one atom per thousand, so these } \\
\text { instruments can quantify concentrations } \\
\text { of the primary elemental constituents } \\
\text { and can map relative abundance of } \\
\text { fission products.However, the } \\
\text { measurements performed in this study } \\
\text { are qualitative only, as only } \\
\text { standardless calibration was used. }\end{array}$ \\
\hline
\end{tabular}

Layer debonding SEM: Identification of macro- and micro-debonding

Electron microscopic technique: Advanced examination (Phase 2)

TEM: Micro and nanostructures will be studied to measure diffraction patterns to identify phase and irradiation damage if any.

\section{TEM}

TEM and TEM-EDS: This examination will reveal more detail of the nature and location of the precipitate, e.g. if the precipitate consists of multiple phases, the main elemental constituents, and if it is located on grain boundaries or transgranular.

Advanced microscopy techniques*: Only selected samples may be analyzed for quantitative results at the atomic level to resolve small quantities of $\mathrm{Ag}$ in the precipitates

TEM: Interlayer properties will be studied on samples at the submicron and nano-level 


\begin{tabular}{lll}
\hline \multicolumn{1}{c}{ Objective } & \multicolumn{1}{c}{$\begin{array}{c}\text { Electron microscopic technique: } \\
\text { Basic examination (Phase 1) }\end{array}$} & \multicolumn{1}{c}{$\begin{array}{c}\text { Electron microscopic technique: } \\
\text { Advanced examination (Phase 2) }\end{array}$} \\
\hline $\begin{array}{l}\text { Grain size and grain } \\
\text { boundary character }\end{array}$ & $\begin{array}{l}\text { SEM-Electron backscatter diffraction } \\
\text { (EBSD)*: provide data on } \\
\text { crystallographic orientation of SiC } \\
\text { grains and grain boundary character in } \\
\text { order to aid the interpretation of } \\
\text { observed fission product behavior } \\
\text { Fuel kernel porosity }\end{array}$ & $\begin{array}{l}\text { TEM: Grain sizes of single grains will be } \\
\text { measured during TEM analysis, but this will not } \\
\text { represent a statistical approach and is for } \\
\text { information only as it is relevant to a very small } \\
\text { number of grains in a small area of the layers. }\end{array}$ \\
& $\begin{array}{l}\text { done using the SEM, only comparative } \\
\text { micrographs will be produced.* Optical } \\
\text { microscopy measurements will be } \\
\text { conducted to compare the relative } \\
\text { porosity among different kernels. }\end{array}$ \\
\hline
\end{tabular}

\subsubsection{SEM}

The SEM used for PIE of the irradiated AGR fuels is a JEOL-7000F equipped with wavelength dispersive spectroscopy (WDS) and energy dispersive spectroscopy (EDS) detectors. Backscattered electron images and secondary electron images were produced from the samples. The SEM was operated between 10 and $30 \mathrm{keV}$. WDS and EDS mapping were conducted to provide the elemental distribution at selected locations throughout the TRISO fuel. The WDS in particular was performed on the precipitates in the $\mathrm{SiC}$ and IPyC layers in an attempt to determine whether $\mathrm{Ag}$ was present. . There is extensive overlap of U, Pd and Ag peaks in the EDS spectra, leading to uncertainty regarding the presence of $\mathrm{Ag}$ in the precipitates as discussed further in Section 4.1.1. EDS and WDS data for the maps were collected for extended periods of time (mostly overnight) to collect sufficient data from the low concentration of fission products throughout the sample. Spatial resolution in X-ray analyses varies with the material and accelerating voltage, but is estimated at several micrometers

\subsubsection{FIB Tool Description}

The FIB uses both scanning electrons and ions to prepare site-specific cross-section samples for TEM and atom probe tomography. The FEI Quanta 3-D FEG Dualbeam FIB, located in the Electron

Microscopy Laboratory (EML), is capable of preparing samples from irradiated nuclear fuel by using the ion beam to sputter material away, creating thin lamella and leaving distinct features observed in the bulk to remain in the $<100 \mathrm{~nm}$-thick TEM sample. Progressively lower ion beam currents are used to form the electron-transparent sample similar to using progressively finer grit in mechanical polishing, producing little surface damage. Material deposition can be performed in site-specific regions via an ion beamassisted chemical vapor disposition process. Deposited material welds samples to TEM grids and atom probe tomography coupons, and can provide a protective surface layer to minimize milling damage. Micromanipulators allow prepared regions to be plucked from the bulk and welded to any surface at desired locations.FIB sample preparation used $\mathrm{Ga}$ and $\mathrm{Pt}$ and these elements appear occasionally as artifacts in TEM-EDS spectra.

\subsubsection{TEM}

TEM was performed with a JEOL 2010 TEM operating at $200 \mathrm{keV}$ and typical magnifications of 5,000 to $100,000 \mathrm{x}$. This microscope is equipped with a $136 \mathrm{eV}$ Oxford EDS detector. Bright field and 
dark field images were produced in key regions of the samples, focusing on key microstructure features. A double tilt holder was used to tilt the sample to key zone patterns and 2-beam conditions to better characterize the microstructure. Zone patterns helped identify if the sample was crystalline or not, and if crystalline, to characterize the crystal structure. Two-beam conditions were used to image existing defects. Over- and under-focus imaging was used to image gas bubbles in the material.

EDS was used to help identify the chemical composition of the key areas of the sample. The primary features of interest are the precipitates near the SiC/IPyC interface. Spot EDS analysis was performed focusing on what elements are present and their concentration. No EDS maps were performed with the TEM. The spot size of the TEM is roughly $20 \mathrm{~nm}$, making the detailed analysis of very small precipitates difficult. EDS analyses of precipitates are likely to involve some contribution from the surrounding matrix, particularly for very small precipitates.

\subsection{Sample Preparation}

\subsubsection{Particle Mounting, Grinding, and Polishing}

The selected CPs from Compact 6-3-2 were mounted in epoxy inside stainless steel rings in the hot cells at the Analytical Laboratory. Two mounts were created: mount 47T contained CP 30 (high in-pile silver release) and mount 48T contained CP34, CP35, and CP39 (low in-pile release). The mounts were loaded with thin double-sided tape stretched across the bottom opening and then backed with a glass slide. The thin tape could pull inward as the epoxy cured and shrank, so particles were not always aligned with the mount base as intended and they were not always at the same depths. Consequently, the three narrow steps machined along the mount exterior as guides for when to backpot and when midplane depth was reached were not useful. In practice, it was necessary to grind well beyond the steps to reach midplane, which required slow removal of the full 0.25-inch stainless steel wall thickness. Another problem was that the epoxy in these mounts was not heat-cured at the Analytical Laboratory, so particles could retreat further into the mounts after arriving at the containment box.

Preparation of mount 48T was completed in the hot fuel examination facility (HFEF) and was straightforward, primarily because particles were relatively close to the base of this mount. It was ground with a Struers MD-Piano 600-grit disk using 10-20 N of force until kernels were just exposed in two of three particles. At this depth, the buffer-to-IPyC gap from buffer densification was opened in two particles for backpotting to stabilize the kernels and buffer layers. Coarse grinding then continued with a 600-grit disk until two of three particles were approximately at midplane. Fine grinding was then conducted with a MD-Piano 1200-grit disk for 2 minutes using $20 \mathrm{~N}$ of force. Polishing began with a 3-micron diamond suspension on a Struers DAC cloth using $20 \mathrm{~N}$ of force, which continued for a total of 6 minutes until periscope viewing indicated that grinding scratches were no longer conspicuous in the epoxy- $\mathrm{SiC}$ layers became noticeably reflective. Polishing continued with a 1-micron diamond suspension on a NAP cloth for 6 minutes at $20 \mathrm{~N}$, whereupon the epoxy also became shiny. Polishing was completed with a 0.25 -micron diamond suspension on a CHEM cloth, again for 6 minutes at $20 \mathrm{~N}$.

The same approach was attempted on mount 47T. The single particle was initially located deep inside the epoxy, so a substantial amount of stainless steel had to be ground away to expose the particle. The particle was finally exposed and ground until the kernel was exposed for backpotting of the buffer-toIPyC gap. After removal of surplus backpotted epoxy, coarse grinding continued until 47T appeared ready for fine grinding and polishing. Several weeks passed before preparation could resume on 47T. Fine grinding with a 1200-grit disk failed to remove scratches. Careful observation established that the epoxy had retreated into the mount, at which time it was learned that heat-curing had not been performed. Coarse grinding had to be conducted again with a 600-grit disk until sufficient stainless steel was removed to reach the particle. Force was increased to $30 \mathrm{~N}$ to expedite the lengthy process. Unfortunately, the last coarse grind went far deeper into the particle than desired (well beyond midplane), the kernel was 
lost, and breakup of the hard kernel resulted in deep scratches in remaining layers and some fractures in the SiC. Another backpot was performed in an attempt to salvage the remaining layers, especially the SiC. Poor control over grinding depth during removal of surplus epoxy again resulted in removal of far more particle than desired. All that remained was the crown of the $\mathrm{SiC}$ as shown in Figure 5; most of the particle was removed during polishing, leaving only a small portion of the $\mathrm{SiC}$ layer embedded in the epoxy. Note that this particle had previously undergone burn-leach, removing the OPyC layer. Because the plane of the met mount appeared to be very near the location of the IPyC-SiC interface, it was decided that this specimen might still provide useful data on SIC microstructure and fission product inclusions and it was fine ground and polished as described above for 48T.

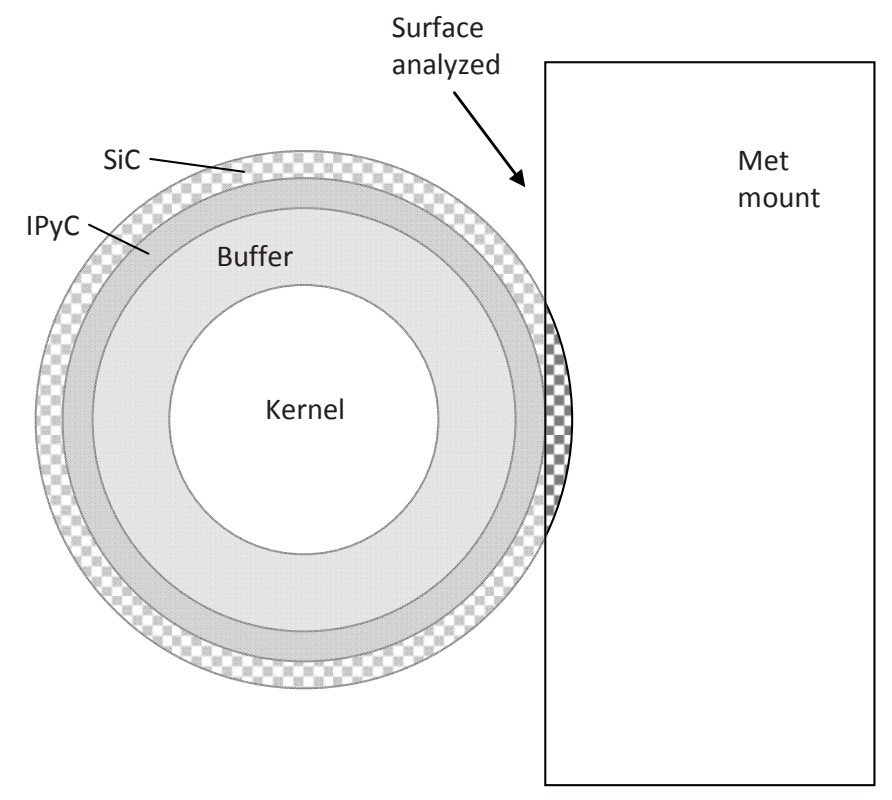

Figure 5. Diagram indicating the state of the met mount of CP30 from Compact 6-3-2.

Difficulties with mount 47T resulted in major changes to the mount design for loose particles. Stainless steel was replaced with Micarta, an internal groove was added to lock epoxy in place near the particle elevation, and the epoxy was cured in a furnace at the Analytical Laboratory. The thin tape was replaced with a thicker, stiff double-sided tape that adhered much better to the glass slide so particles were kept closer to their as-loaded elevations as the epoxy cured.

\subsubsection{Decontamination in EML}

The mounted samples shipped from the HFEF to EML were received in lead containers (pigs). The met mount samples were inside plastic tubes that were cushioned with foam and bagged with the pertinent radiological information and sample IDs. The bagged samples were transferred into the EML actinide glove box along with decontamination supplies where the met mounts were removed from the plastic transfer tubes. The met mounts were then placed on a clean paper towel. The sides and back of the met mounts were initially wiped against 1200 grit polishing paper while being careful not to damage the front of the met mount that contained the sample. This step removed any contamination fixed to the side and back surfaces of the met mount. The sides and back were then wiped down with paper towels that had been soaked with various cleaners. The first paper towel was soaked with glass cleaner. This was followed by different paper towels soaked with deionized water, acetone, and methanol. The entire met mount, including the front, was then rinsed with acetone followed by a rinse with methanol. At this point, the met mount was checked for its contamination levels. If the contamination levels were low enough to 
remove the sample from the glove box, the samples were capped and transferred from the glove box to the radiological chemical hood. If the contamination levels were too high to remove from the glove box, the decontamination procedure was repeated until the sample was clean enough to remove. The samples in the radiological chemical hood are placed face down on a clean paper towel for spray painting. This step serves to fix any contamination that is left on the mount into the paint so that the mount will survey free of any removable contamination. Several layers of spray paint were added to the met mount until the removable contamination was low enough to allow the sample to be removed from the hood. The sample was then transferred to the Hummer Sputter Coater to put a thin conductive layer on the sample of approximately $30 \mathrm{~nm}$ of gold. The sample was then placed in storage until researchers were ready to insert the sample into the SEM. 


\section{RESULTS AND DISCUSSION}

The analysis focused primarily on CP34 and CP35 from mount 48T and CP30 from mount 47T. CP39 from mount 48T was found to have significant debris on the surface, most likely from the preparation and cleaning steps, which hindered detailed analysis of certain microstructural features. Results for each individual $\mathrm{CP}$ are provided in this section with the integration of the results in Section 6.

\section{$4.1 \quad$ CP34}

\subsubsection{SEM Examination}

\subsubsection{Interlayer Thickness and Pd Precipitate Distribution Determination}

The JEOL 7000 FEG SEM was used to measure the interlayer thickness and precipitate distribution in CP34 from mount 48T. An image montage was made by capturing sequential backscattered electron micrographs of the IPyC/SiC interface at $4000 x$ magnification, making sure that there is adequate overlap between adjacent images to stitch them together. Adobe Photoshop was used to combine the 81 images by overlapping shared features within images to form a complete image montage of the particle interface.

From each individual image, the interlayer thickness was measured from the $\mathrm{SiC}$ finger that extended the furthest into the IPyC to the portion of the $\mathrm{SiC}$ that was furthest away from the interface, measured perpendicular to the interface as shown in Figure 6. This method provided more than adequate statistics as presented in Figure 7. This method may yield different interlayer thickness values depending on the image sizes (because of the different length of the interlayer used for measurement), but for the CP34 specimen, the evaluation is standardized to use images obtained at the same magnification. It is important to note that no standardized method to measure the IPyC-SiC interlayer exists in the HTR research community, and this method was used for this evaluation as it provides a repeatable method for comparison purposes. As this montage was prepared after FIB sample preparation because of scheduling conflicts on SEM, FIB and operator availability, three areas were not included in this evaluation. However, based on the presentation of the results, it is clear that these missing results did not compromise the final conclusion of this work. The data from Figure 7 showed that the IPyC-SiC interlayer thickness varies from 0.65 to $3.69 \mu \mathrm{m}$, with an average thickness of $1.73 \mu \mathrm{m}$. 


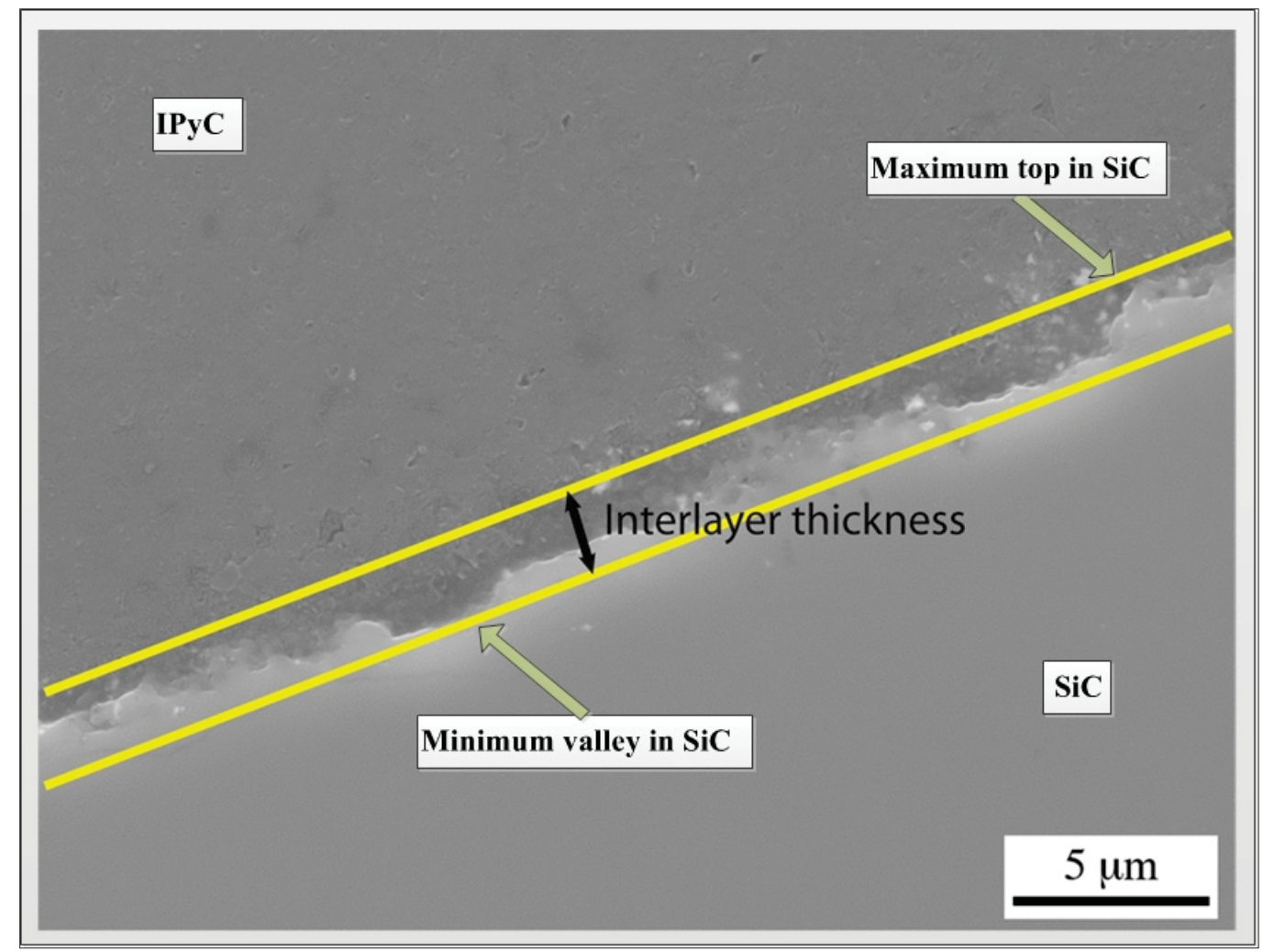

Figure 6. Interlayer thickness measurement schematic presentation.

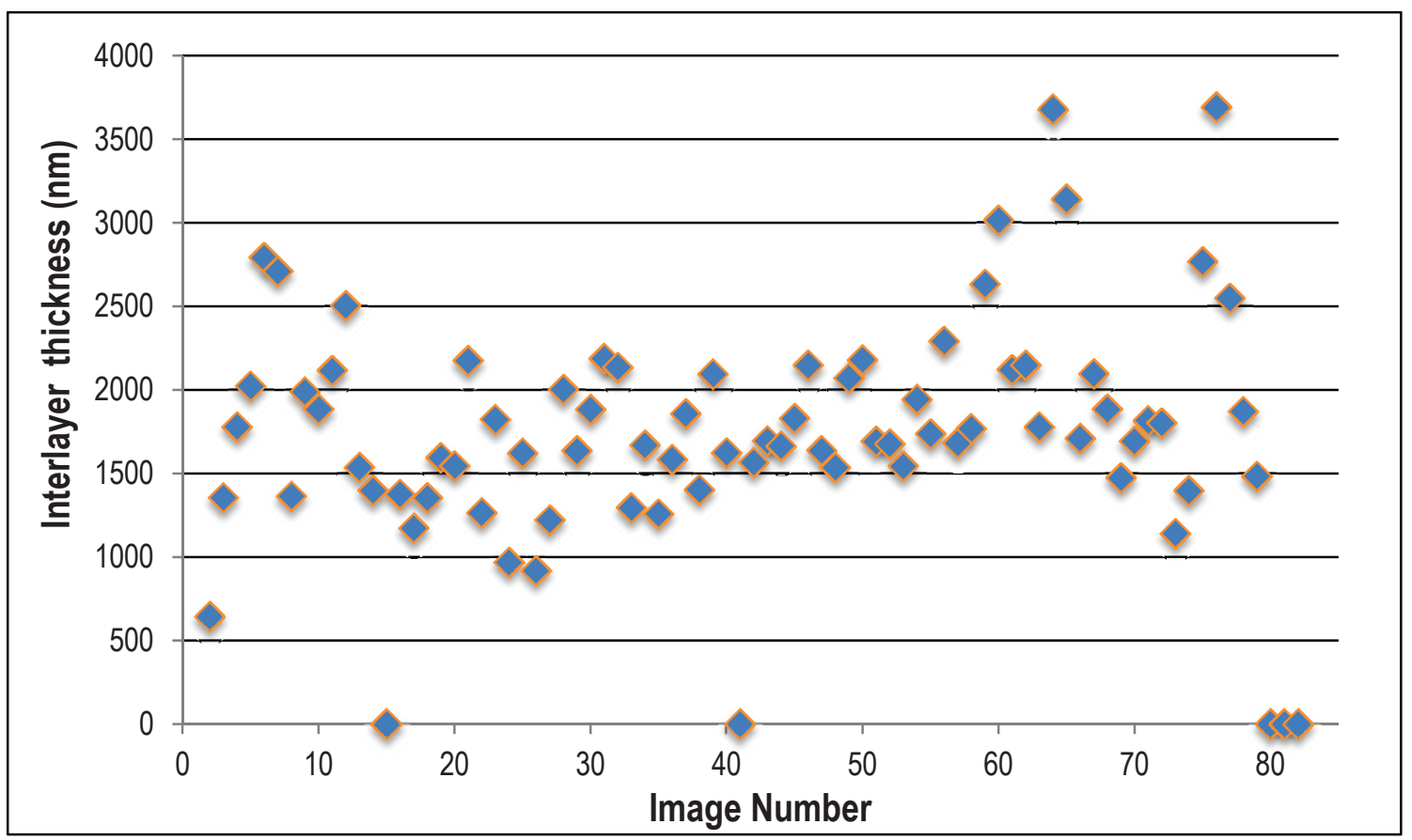

Figure 7. IPyC-SiC interlayer thickness measured from SEM montage (zero values in locations where FIB sample preparation was completed). 
The distribution and position of the precipitates in the coating layers are also of interest as this may provide insight to the transport behavior of some of the fission products. Therefore two measurements were performed and are presented here. Precipitate identification around the entire particle was identified based on the light contrast items on backscattered SEM images in the coating layers because initial SEM EDS analysis of specific spots in similar images indicated the presence of fission product precipitates. Thus, not all the precipitates in the circumference were identified based on EDS or WDS elemental analysis.

Similar to the interface determination, the distance of the furthest precipitate from the interface in both $\mathrm{SiC}$ and PyC layers was measured for each image as schematically demonstrated in Figure 8. The results shown in Figure 9 indicate that the precipitates are present in the $\mathrm{SiC}$ layer up to an average depth of $6.74 \mu \mathrm{m}$ but with a maximum dept of $15.25 \mu \mathrm{m}$. The measurements in the IPyC revealed that most of the precipitates are located within $2.9 \mu \mathrm{m}$ of the IPyC-SiC interface with a maximum distance $15.25 \mu \mathrm{m}$, suggesting that the interface and/or SiC-layer acts as an initial barrier to the transport of these precipitates.

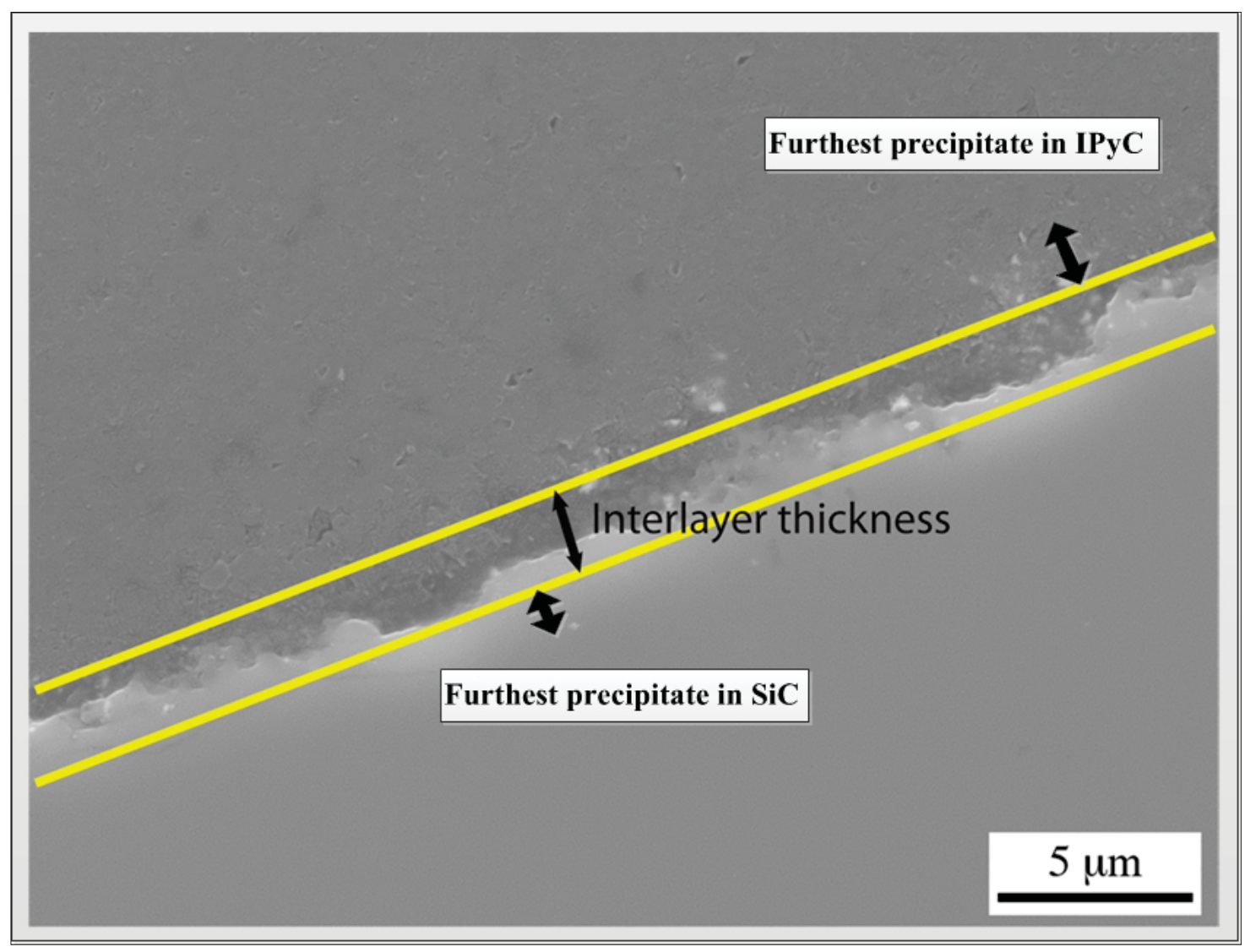

Figure 8. Schematic presentation to show measurement of furthest precipitate from Interlayer.

As a first order estimate to determine the amount of precipitates and distribution of precipitates in each layer, the number of particles in each image was also counted and graphically presented in Figure 10. The average number of precipitates identified in the IPyC and SiC layers are similar $(\sim 24$ precipitates on average per image), although the highest number of precipitates (70) in a single image were observed in the IPyC layer, compared with only 45 precipitates in the $\mathrm{SiC}$ layer. No specific trend is observable in the circumferential distribution of these precipitates. As this was the first generation of this type of analysis, the value of quantifying the abundance and distribution of precipitates in this manner 
was uncertain, but it was speculated that it may provide insights into the morphology of precipitates and/or the pathway of the fission products in the coating layers. Based on lessons learned, image analysis techniques may be considered for future precipitate maps. Compiling all data in Microsoft Excel showed that the distribution appears to be random over the entire particle interface circumference. Further investigation revealed that clusters of precipitates were noted. The criteria for cluster identification at this point are not quantifiable and are based only on the visual groupings of precipitates. Clusters were then treated as a single feature, but also yielded random distributions as shown in Figure 11. In summary, (a) precipitates were randomly distributed around the circumference of the particle, (b) more precipitates were found in the IPyC layer than within the $\mathrm{SiC}$ layer, and (c) some clustering of precipitates was observed but this was also random.

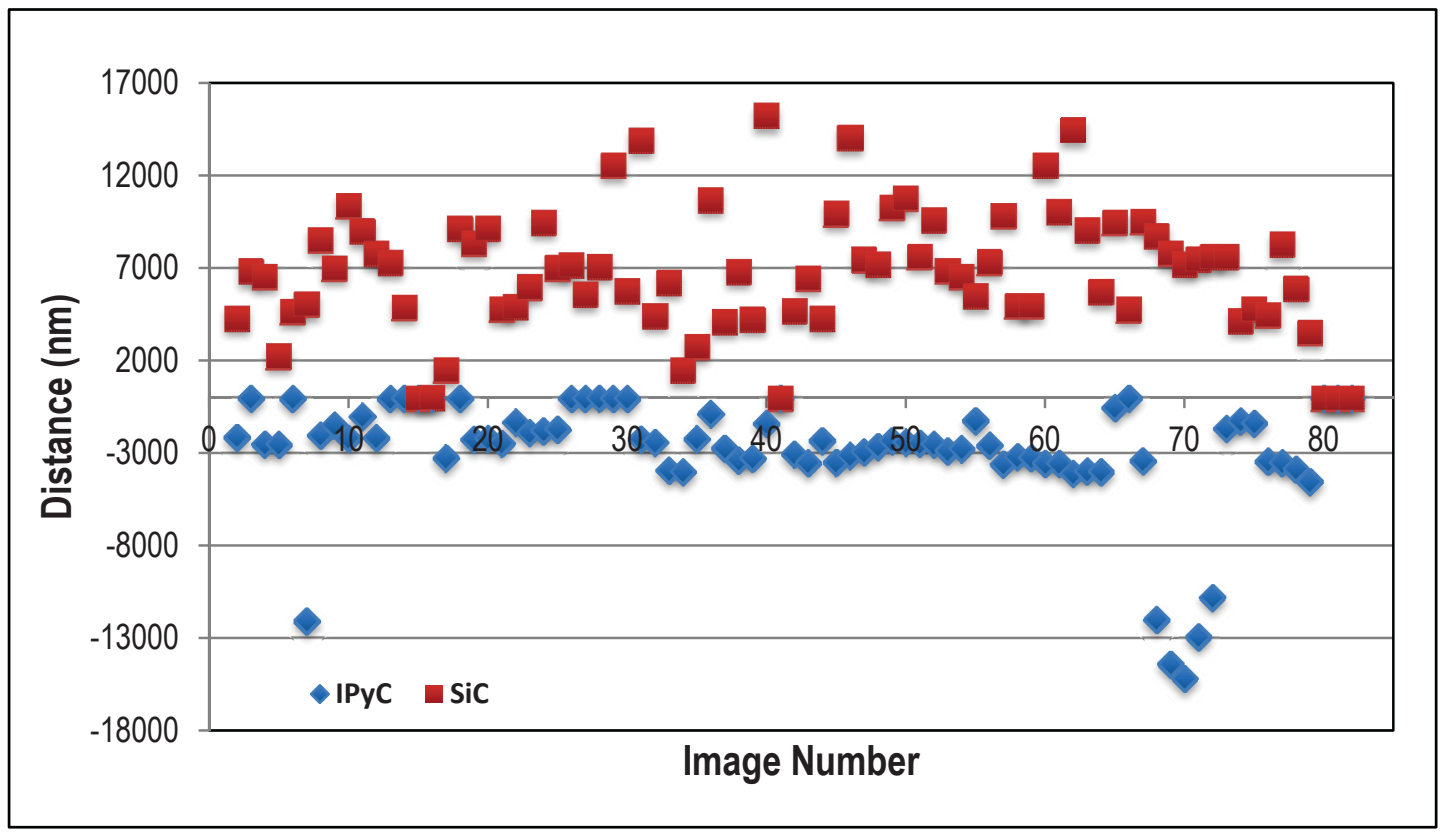

Figure 9. Precipitate position as a function of the distance away from the IPyC-SiC interlayer. 


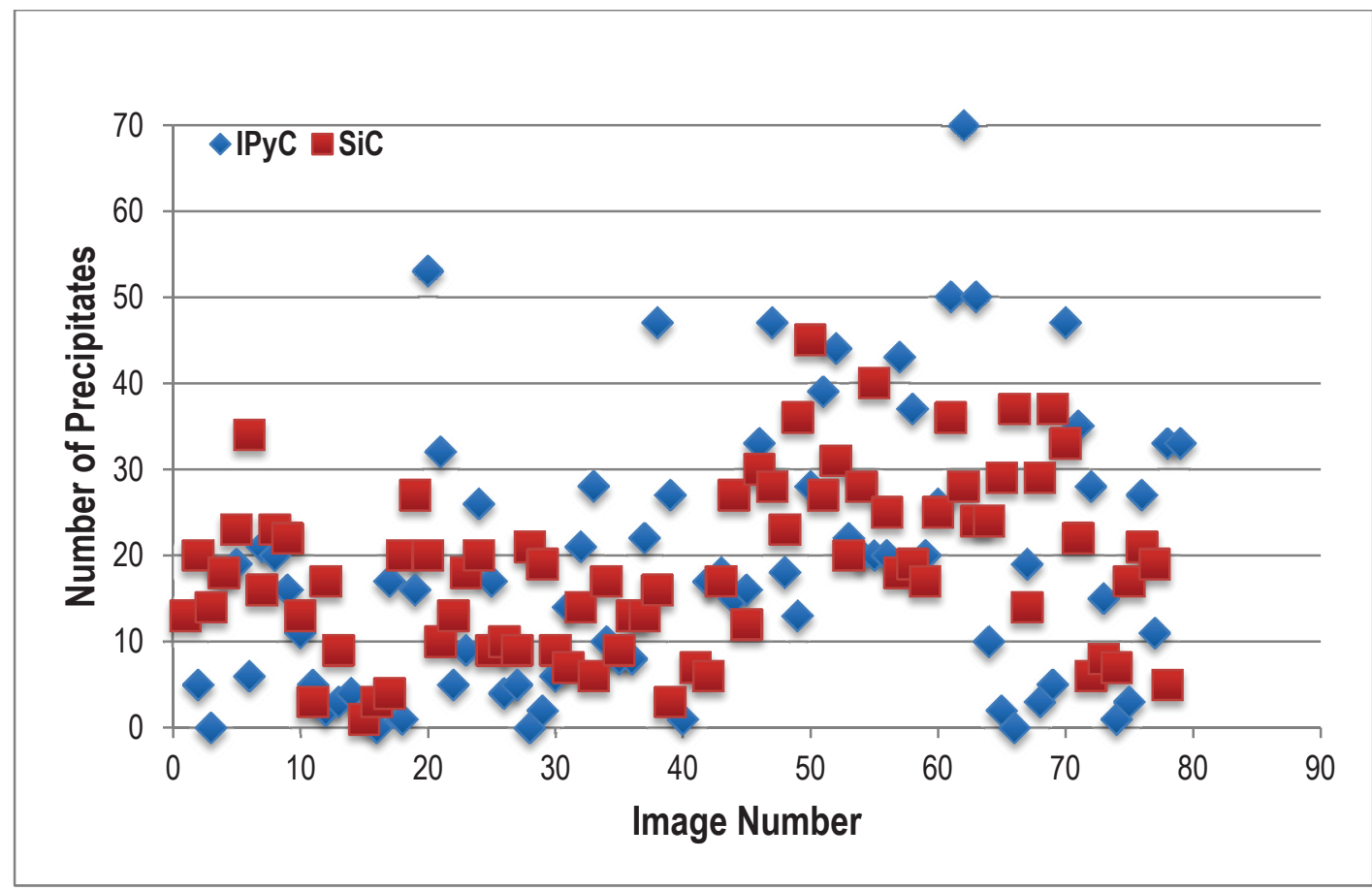

Figure 10. Number of precipitates located in the SiC and IPyC layers of CP34 based on SEM montage.

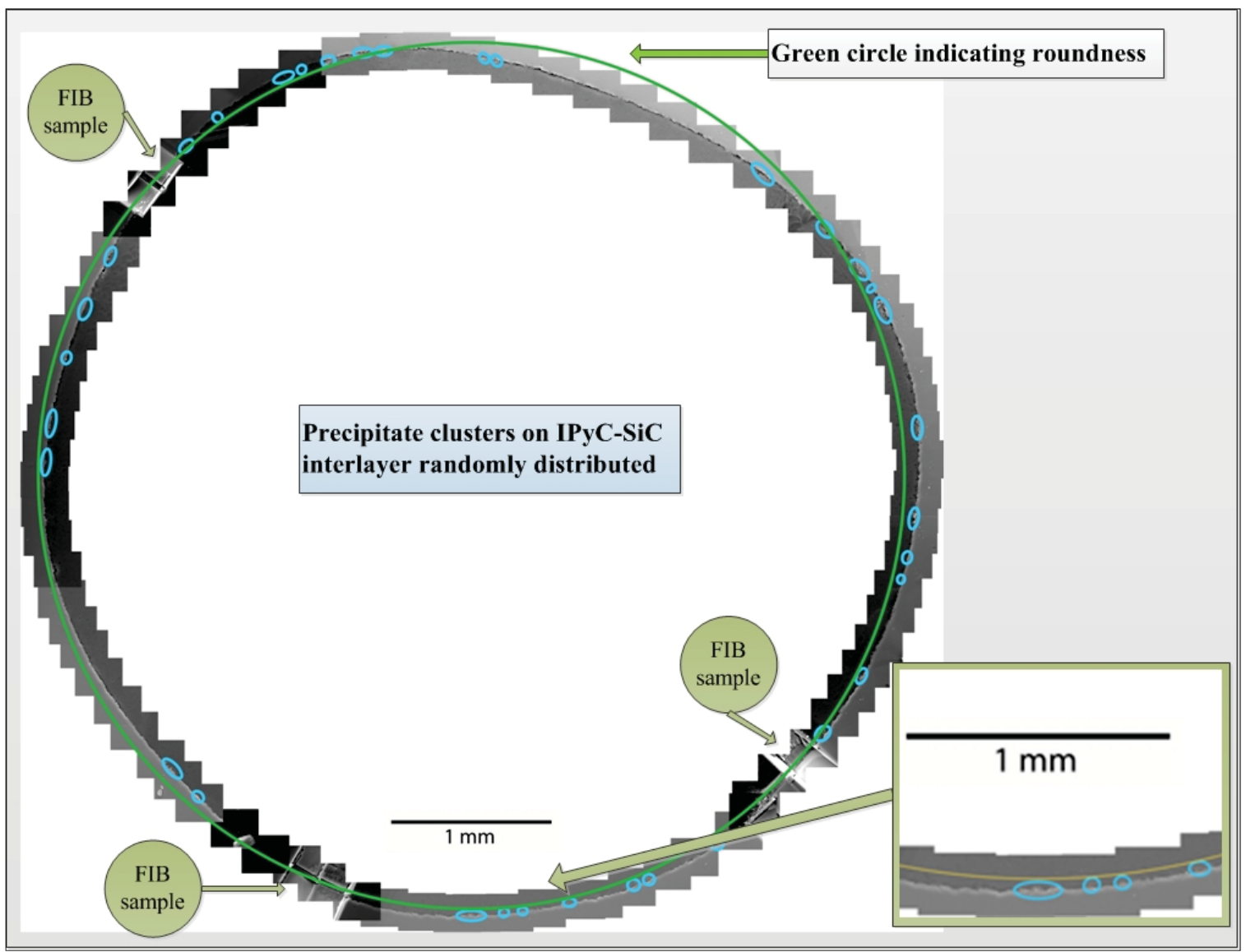

Figure 11. CP34 SEM montage showing randomly distributed precipitate clusters encircled by blue. 


\subsubsection{SEM Precipitate Identification}

The SEM plan shown in Figure 3 in Section 3.2.1 was used for the initial investigation with the exclusion of the electron backscatter diffraction (EBSD) measurements. The initial EDS examination focused on $\mathrm{Cs}, \mathrm{Ag}, \mathrm{Pd}, \mathrm{Zr}, \mathrm{Rh}$, and Ru, identified in the locations marked 1 and 2 in CP34 shown in Figure 12. Palladium-rich precipitates are fairly homogenously spread around the circumference at the SiC-IPyC interface as discussed in Section 4.1.1.1. Interestingly, the Pd precipitates in location 1 seem to be situated in possible SiC "fingers" while the Pd-precipitates in location 2 are visible in the IPyC as well and not only on the SiC fingers, as shown in Figure 13 (taken from the EDS maps). Characterization studies by Barrachin et al. ${ }^{3}$ on HFR-EU1bis experiments (10.2\% FIMA, calculated pebble center temperature of $1523 \mathrm{~K}$ ) found that Pd is specifically present in the IPyC layer at the inside of the SiC layer, but no Pd is mentioned to be found in the SiC layer itself. This study on CP34 shows, in contrast with the finding of Barrachin et al. ${ }^{2}$, that $\mathrm{Pd}$ precipitates are also present inside the SiC layer as is shown in Figure 14. The precipitates will also be further examined by TEM for composition, location in grain boundaries, and fission product behavior.

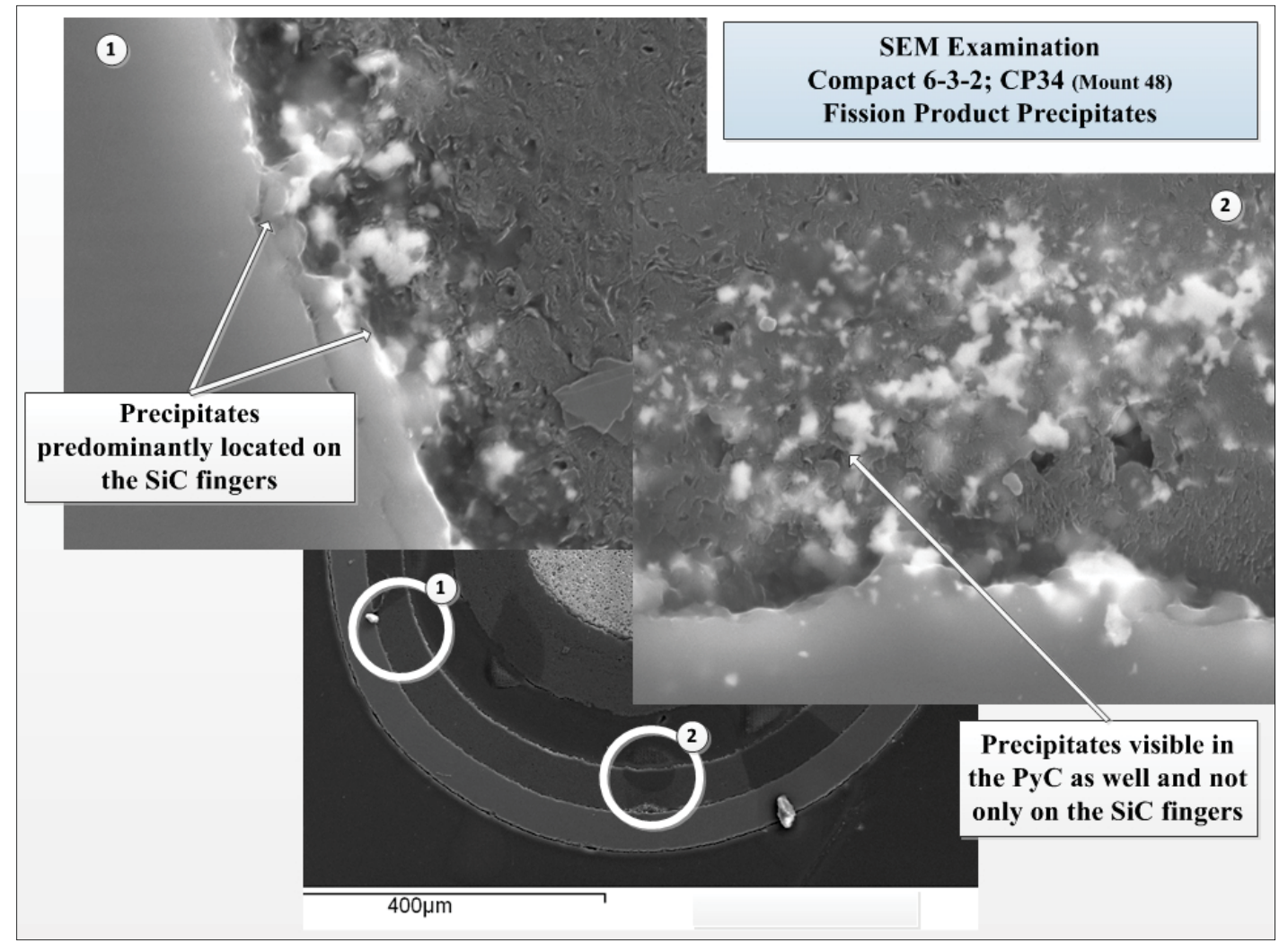

Figure 12. Pd rich precipitates from two different locations showing precipitates located both on the SiCfingers as well as in the PyC near the IPyC-SiC interlayer self. 


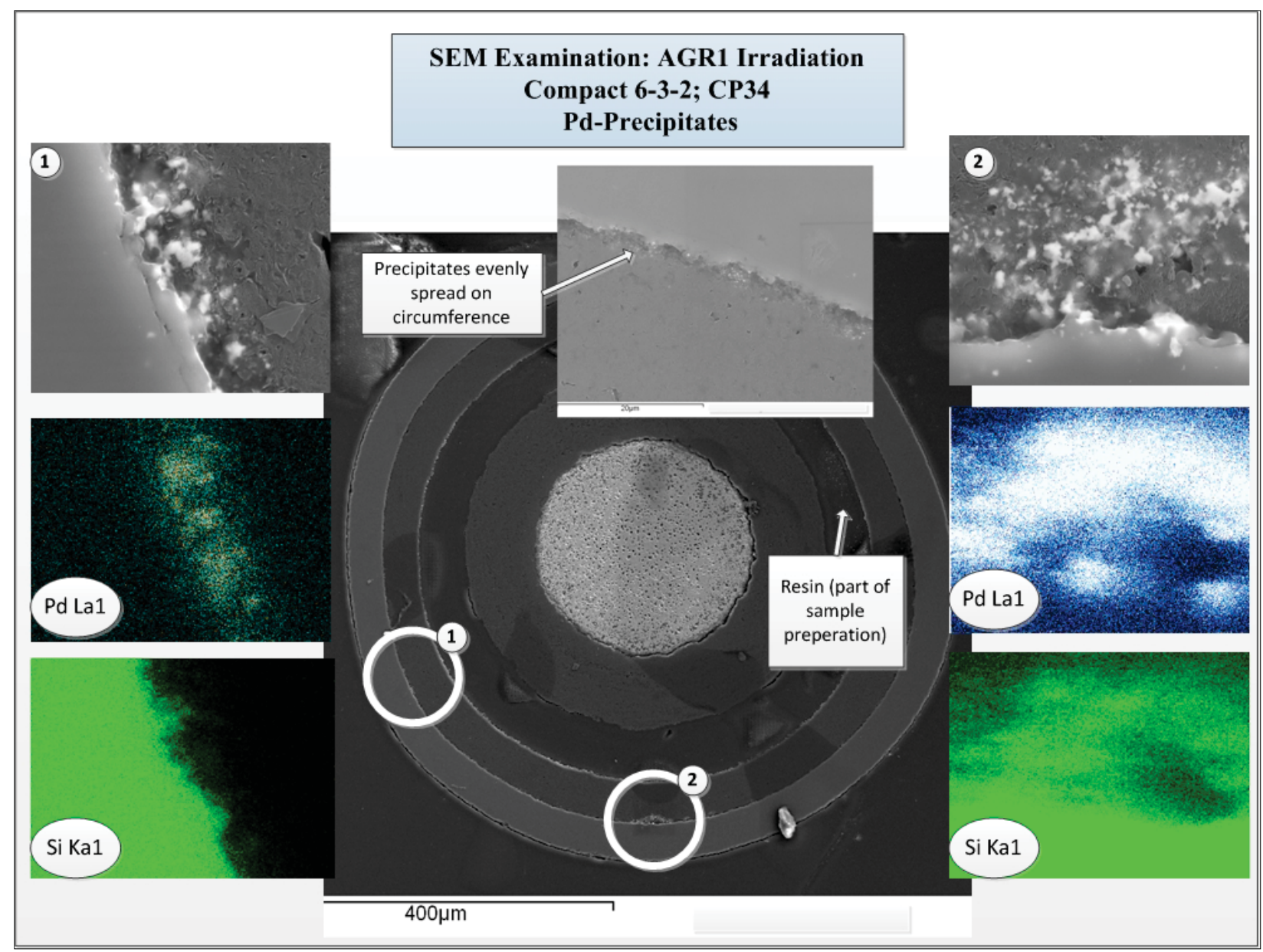

Figure 13. SEM and EDS analysis of CP 34 of Compact 6-3-2, revealing the Pd-containing precipitates in both the SiC and IPyC layers.

While most of the preliminary elemental examinations of these particles used EDS, some analysis was performed using WDS in order to better resolve x-ray energies from specific elements and verify that the EDS spectral analysis was not resulting in misidentification of selected elements. Table 2 and Figure 15 show peak overlaps that are relevant in determining whether low concentrations of Ag are present in EDS spectra from areas with much higher concentrations of $U$ and Pd. All of the Ag peaks within the standard energy range of the current EDS detector on the SEM and TEM (0 to $20 \mathrm{keV})$ are overlapped by peaks from $\mathrm{U}$ or Pd, making it impossible to tell whether Ag is present. If the EDS detector is configured for an expanded energy range (e.g., 0 to $40 \mathrm{keV}$ ), the $\mathrm{Ag} \mathrm{K \alpha l}$ peak can be used to confirm the presence of Ag. This peak may overlap the lower-intensity Ag Ka2 peak (at $21.993 \mathrm{keV}$ ), but does not overlap significant peaks from other elements believed to be present in the sample $(\mathrm{C}, \mathrm{Si}, \mathrm{U}, \mathrm{Pd}, \mathrm{Zr}$, and $\mathrm{Pu})$. 


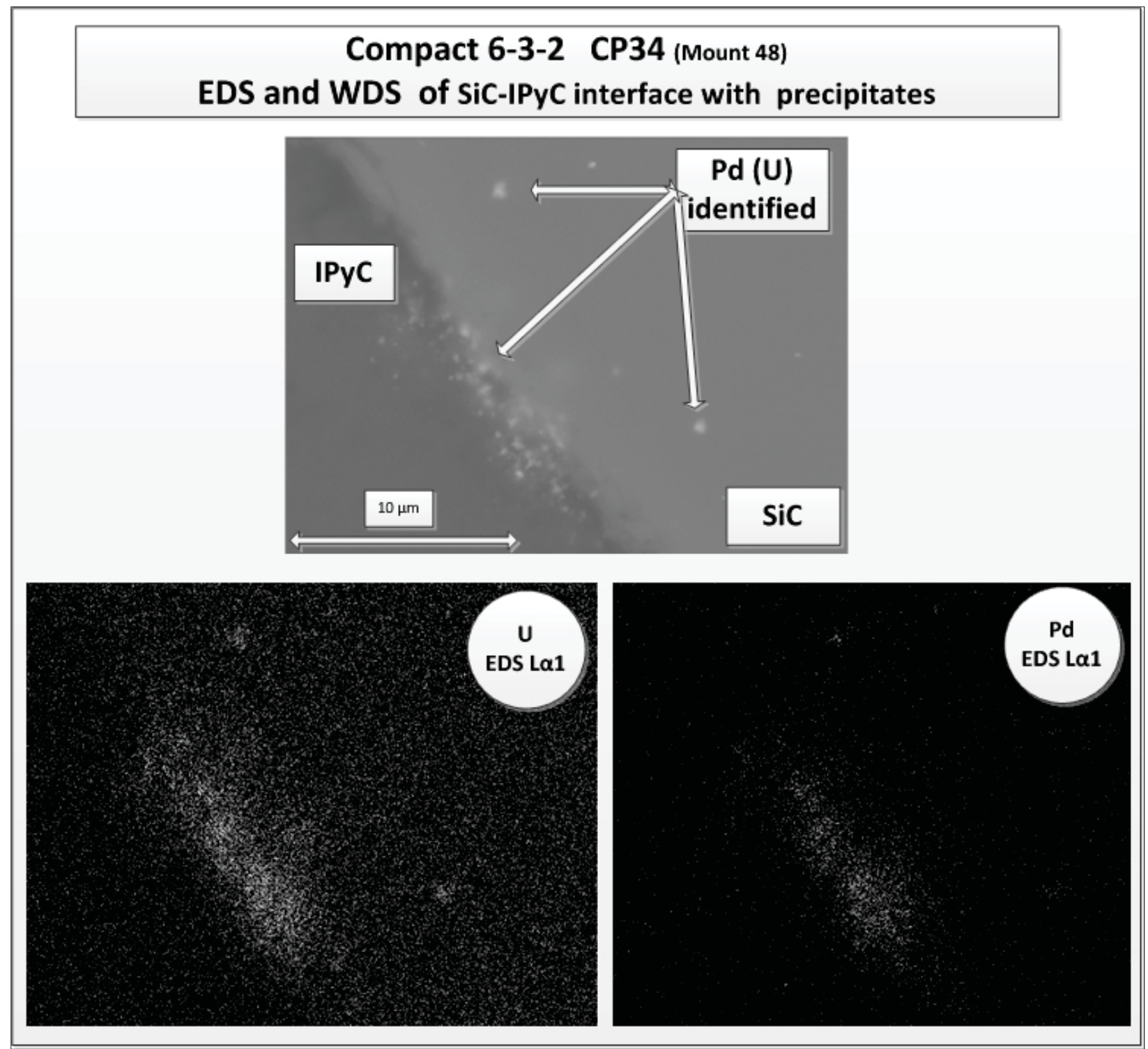

Figure 14. SEM, EDS and WDS analysis of CP 34 of Compact 6-3-2, revealing the cluster of Pd precipitates on the SiC-IPyC interface as well as more discrete Pd precipitates in the SiC layer itself (these Pd precipitates may also contain $\mathrm{U})$. 
Table 2. Relevant X-rays for qualitatively identifying low concentrations of Ag in spectra from areas with much higher concentrations of Pd and U. 4,5

\begin{tabular}{|c|c|c|c|}
\hline $\mathrm{x}$-ray & Energy $(\mathrm{keV})$ & Relative Intensity $^{\mathrm{a}}$ & Overlapped By \\
\hline $\operatorname{Ag~L} \ell$ & 2.633 & 4 & 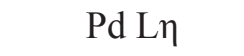 \\
\hline $\operatorname{Ag} \operatorname{L} \alpha$ & 2.984 & $111^{\mathrm{b}}$ & $\operatorname{Pd} \mathrm{L} \beta$ \\
\hline Ag L $\beta 1$ & 3.150 & 56 & $\mathrm{U} \mathrm{M} \alpha$ \\
\hline Ag L $\beta 2,15$ & 3.347 & 13 & $\mathrm{U} \mathrm{M} \beta$ \\
\hline Ag L $\gamma 1$ & 3.519 & 6 & $\mathrm{U} \mathrm{M} \gamma$ \\
\hline $\operatorname{Ag~K\alpha 1}$ & 22.166 & 100 & (None) \\
\hline
\end{tabular}

a Intensity relative to the largest peak in the series, e.g., $\mathrm{Ag} \mathrm{L} \beta 1$ has about $50 \%$ of the intensity of Ag L $\alpha$. No relationship between the intensities of peaks in different series (e.g., $\mathrm{Ag} \mathrm{K \alpha} 1$ and $\mathrm{Ag} \mathrm{L} \alpha$ ) is implied.

b Combined intensity of $\mathrm{Ag} \operatorname{L} \alpha 1$ and $\mathrm{Ag} \mathrm{L} \alpha 2$, which overlap each other.

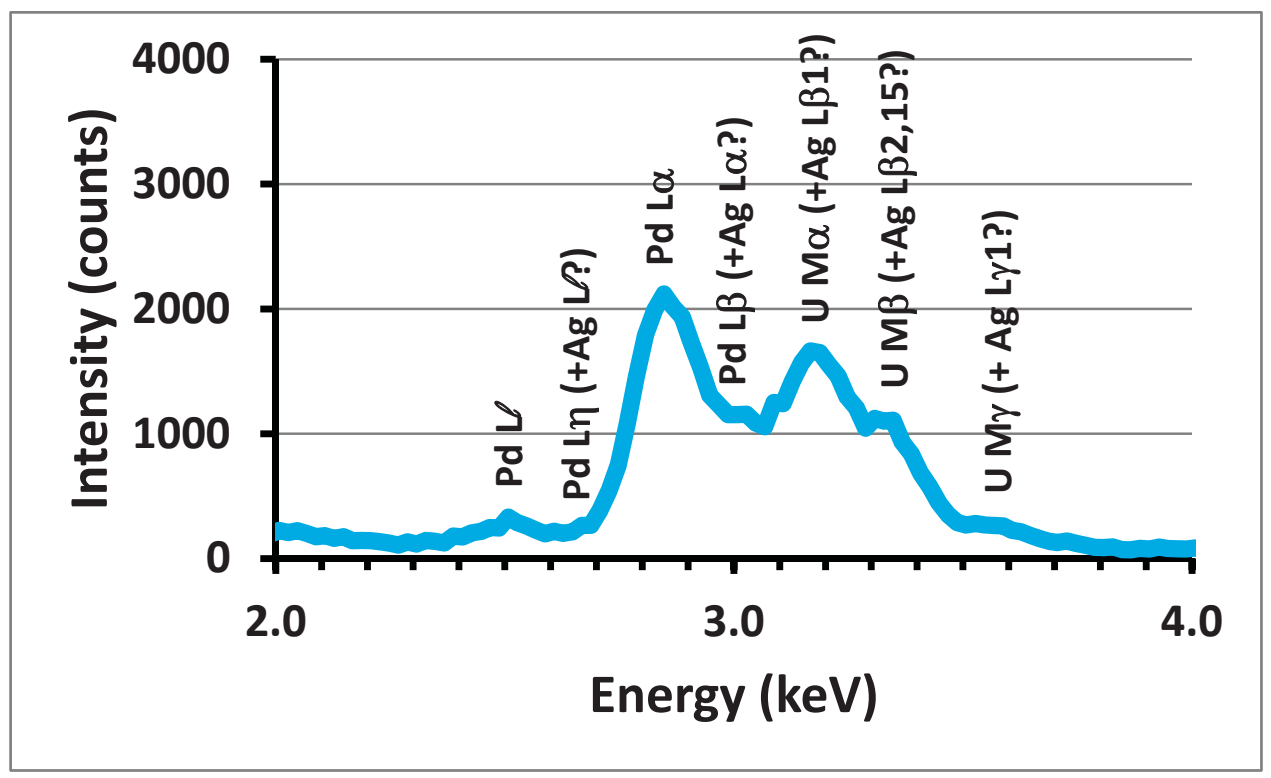

Figure 15. Typical expanded section of EDS spectrum showing details of overlaps between $\mathrm{Ag}, \mathrm{Pd}$, and $\mathrm{U}$ (EDS spectrum taken from CP30 picture 13 in original EML database). See Table 2 for energies for Ag $\mathrm{x}$-rays.

Although it may be possible in principle to qualitatively confirm the presence of low concentrations of $\mathrm{Ag}$ in SEM-WDS maps from areas that have much higher concentrations of $\mathrm{Pd}$, it is important to select the Ag x-ray carefully. For example, the energies of the Ag L $\alpha 1$ and Pd L $\beta 1$ x-rays $(2.984$ and $2.990 \mathrm{keV}$ respectively) are similar enough that careful analysis is needed to determine whether WDS maps using $\mathrm{Ag} \mathrm{L} \alpha 1$ x-rays really represent only Ag, or also include some contribution from Pd. Despite its lower relative intensity, the Ag L $\beta 1 \mathrm{x}$-ray is more suitable for WDS maps because it is separated by $78 \mathrm{eV}$ from the closest Pd x-ray (Pd L $\beta 3$, at $3.072 \mathrm{keV})$. However, it is only $9.6 \mathrm{eV}$ from the closest U X-ray (U M $\alpha 2$, at $3.1596 \mathrm{keV}$ ). WDS detectors may be able to distinguish between peaks that are $\sim 5-10 \mathrm{eV}$ apart, depending on the relative proportions of the elements present in the sample.

The Ag-WDS results originally collected for this study up to December 2011 were obtained mainly using the Ag L $\alpha 1$ x-rays only, as confirmation of the presence of Ag using the Ag L $\beta 1$ x-rays was delayed by equipment operational problems that were resolvedearly in 2012. However, these WDS examinations 
were also inconclusive for the identification of Ag and further analysis with advanced microscopic techniques like EELS and LEAP needs to be considered.

The SiC layer and SiC-IPyC interface of the first particle met mount (mount 48T) was examined by tilting the sample to examine the amount of relief on the polished particle surfaces. From Figure 16, it is deduced that there is some rounding of the $\mathrm{SiC}$ layer in a $\sim 2 \mu \mathrm{m}$ region near the IPyC interface, which may affect the EBSD results. Based on these results, the sample polishing procedure has been refined to improve the surface finish on future particle mounts and enable quality EBSD data to be acquired. This will be discussed in future reports.

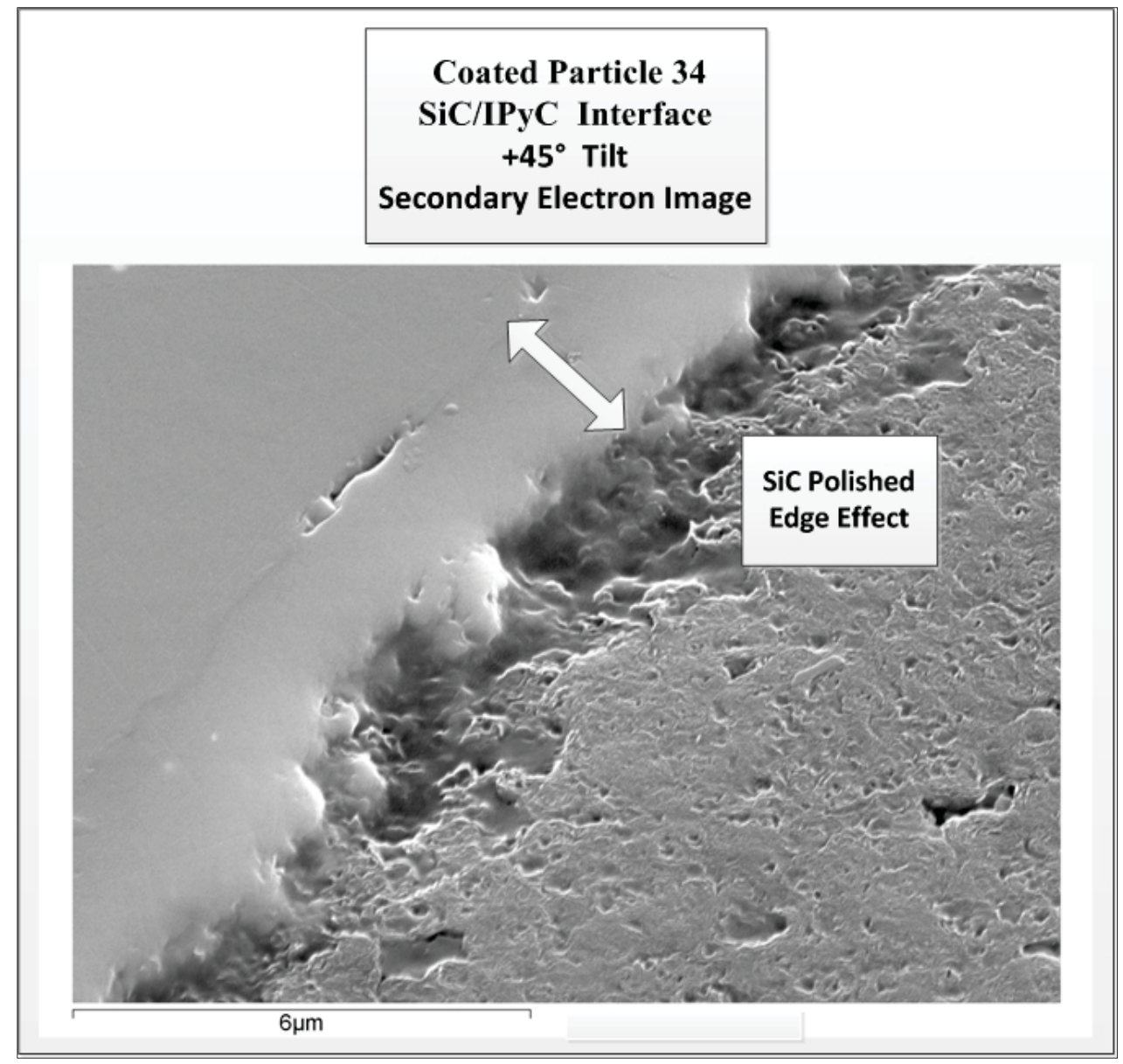

Figure 16. Secondary electron image showing the edge effect because of polishing resulting in an approximately $2 \mu \mathrm{m}$ region of significant relief in the SiC layer.

\subsubsection{FIB Sample Preparation}

FIB specimens have been prepared from CP34 and identified by the numbers shown in Figure 17. Figures 17, 18, and 19 show regions of each particle where TEM specimens were removed with the FIB. Note that a specimen was not prepared from Position 4, but additional specimens were prepared from Position 2.

As seen in Figures 18 and 19, the specimens are thin planar monoliths cut perpendicular to the polished particle surface. Specimens 1 and $2 \mathrm{a}$ were oriented tangent to the SiC-IPyC interface and TEM Specimens 2b, 2c and 3 were taken perpendicular to the coating layers at the SiC-IPyC interface in a 
region where significant numbers of Pd-rich precipitates were found with SEM/EDS analysis (see Figure 18 and Figure 19). The rationale for Specimens 2a, 2b, and 2c was to enable a TEM analysis of the precipitates through the full width of the $\mathrm{SiC}$ layer to provide information at a higher resolution than what is available with the SEM precipitation map. Although it was discovered based on the SEM evaluation that the precipitates are present in the $\mathrm{SiC}$ layer up to a maximum depth of $15.25 \mu \mathrm{m}$, it is important to evaluate this finding at the higher magnification that the TEM analysis can provide. These samples were prepared from an area where precipitate clusters were identified during the SEM evaluation.

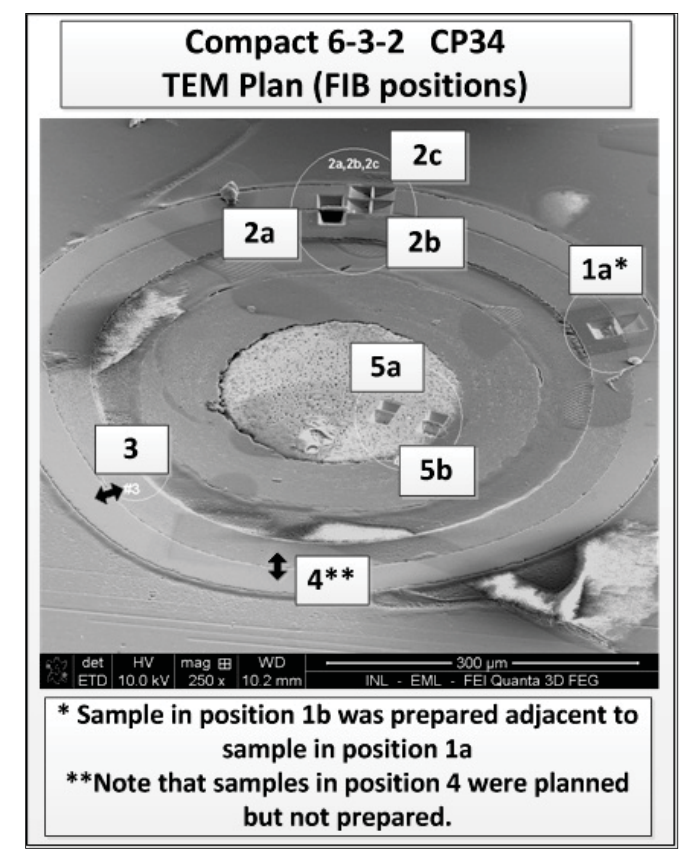

Figure 17. FIB sample preparation position for CP34 from Compact 6-3-2. 


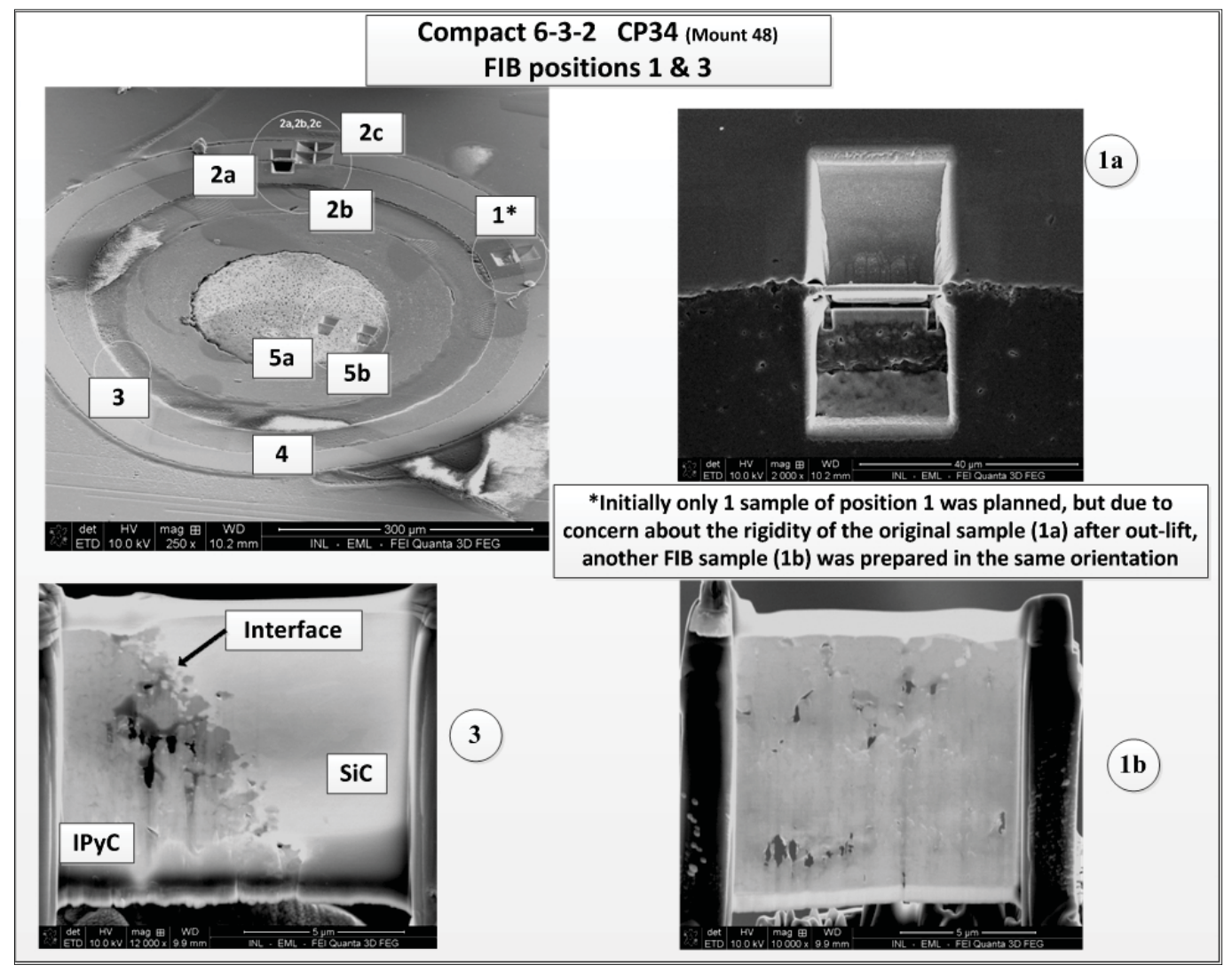

Figure 18. FIB samples for Positions 1 and 3 of CP34. 


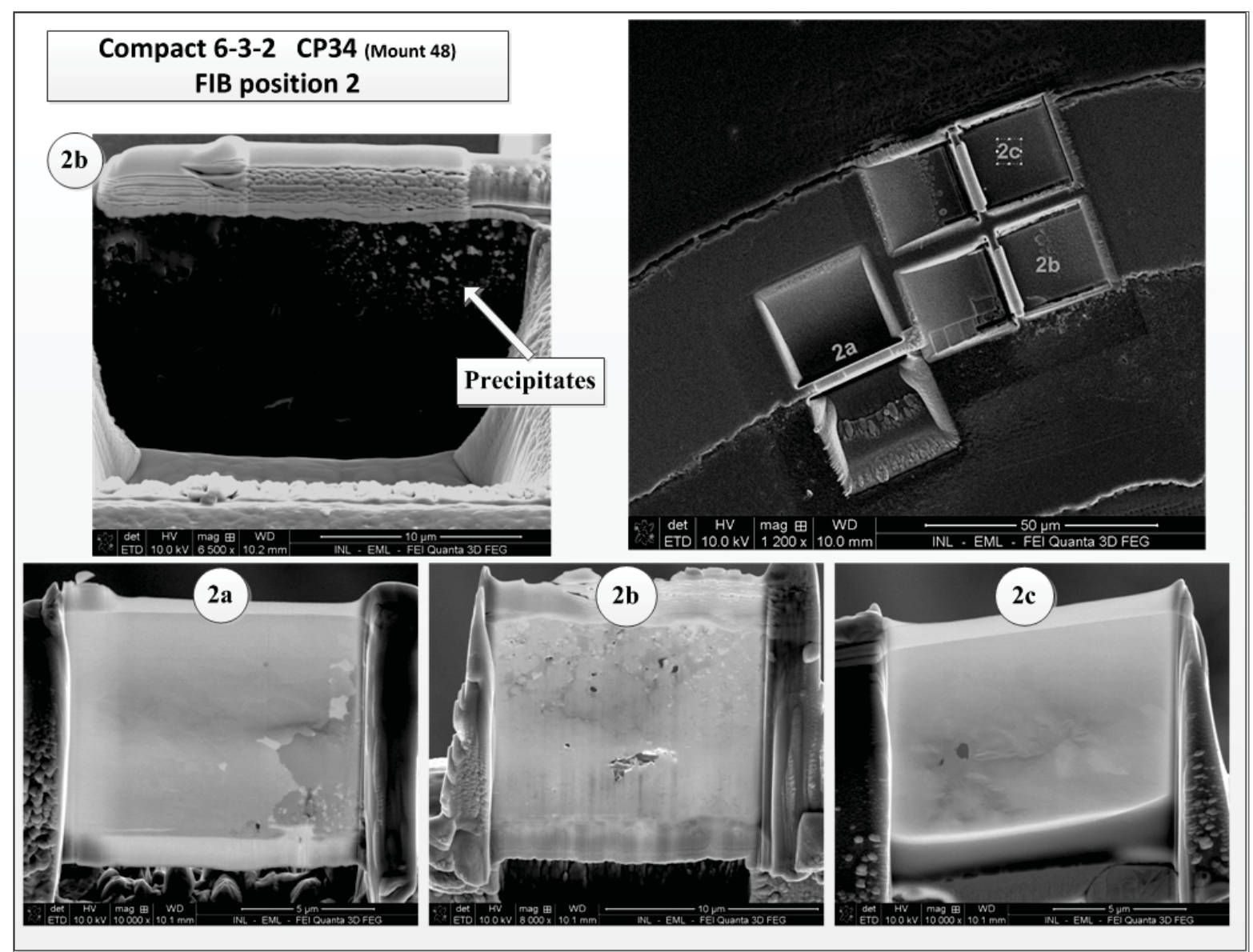

Figure 19. FIB samples prepared at Position 2 of CP34 showing location of sample as well as precipitates clearly visible in the SiC matrix.

\subsubsection{TEM Examination}

The TEM analysis focused primarily on precipitate identification in the IPyC and $\mathrm{SiC}$ layers, $\mathrm{SiC}$ phase identification, and irradiation effects on the IPyC and $\mathrm{SiC}$ layers. The $\mathrm{SiC}$ grain size can also be determined from the selected areas investigated.

\subsubsection{Initial TEM Analysis of CP34}

The initial TEM analysis of the specimens taken from this particle has focused on fission product precipitates in the various coating layers. Figure 20 shows a TEM micrograph with representative images of precipitates in the IPyC layer of CP34 from Compact 6-3-2, TEM Position 1a. This specimen was taken from the IPyC-SiC interface, roughly tangent to the interface.

$\mathrm{Pd}, \mathrm{U}$ and $\mathrm{Si}$ were identified in spectra from these precipitates using TEM-EDS. Small peaks from $\mathrm{Pu}$ were visible in almost all EDS spectra from Pd-rich precipitates from CP34 and CP35. Detection of Pu was complicated by a peak overlap between the Pu L $\alpha$ and $\mathrm{Sr} \mathrm{K} \alpha$ peaks, which was resolved by identification of the $\mathrm{Pu} \mathrm{L} \beta$ peak. Detection of $\mathrm{Pu}$ was also complicated by the small sizes of all of the $\mathrm{Pu}$ peaks, and by the low numbers of counts in many of the spectra. More detail on this confirmation of $\mathrm{Pu}$ presence, in Section 4.1.3.3. 
Figure 21 shows a higher magnification micrograph of a Pd-rich precipitate within a $\mathrm{SiC}$ grain of CP34 from Compact 6-3-2, TEM Position 1b showing nanometer-sized contrast variation. This area was identified during the preliminary SEM/EDS analysis as containing Pd-rich precipitates.

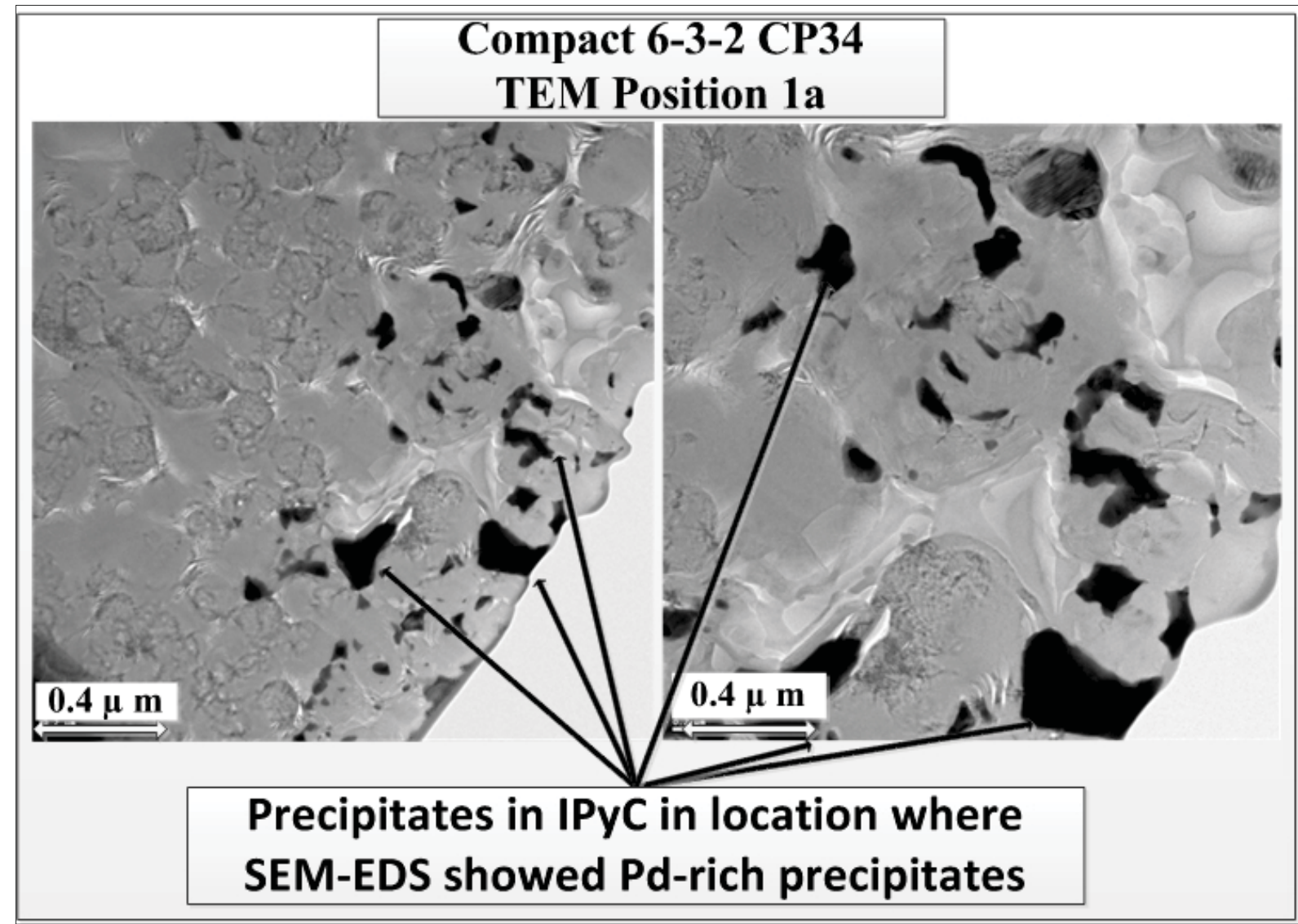

Figure 20. TEM micrograph showing representative images of precipitates in the IPyC layer of CP34 from Compact 6-3-2, TEM Position 1a. 


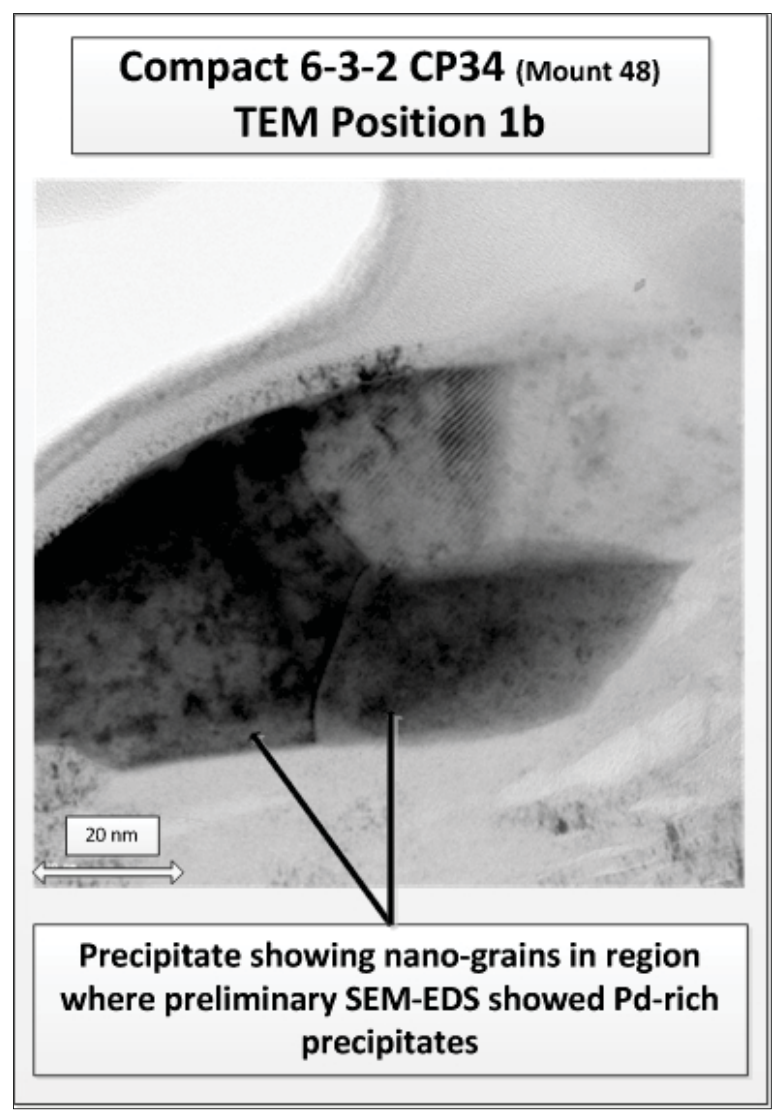

Figure 21. TEM micrograph showing a precipitate with nanometer-sized grains in CP34 from Compact 6-3-2, TEM Position $1 b$.

As mentioned before, the rationale for preparing FIB specimens $2 a, 2 b$, and $2 c$ was to enable a TEM analysis of the precipitates through the full width of the $\mathrm{SiC}$ layer to provide information at a higher resolution than with SEM precipitation map (Section 4.1.1.1). The TEM micrographs for Specimens 2a, $2 \mathrm{~b}$, and $2 \mathrm{c}$ in Figure 22 show that precipitates are visible in Position $2 \mathrm{a}$ and $2 \mathrm{~b}$, but no precipitates were observed at Position 2c. Position 2c, which is on the outer edge of the SiC layer, includes roughly the outer half of the $\mathrm{SiC}$ layer from the midpoint, $\sim 17 \mu \mathrm{m}$ from the IPyC-SiC interface, to the SiC-OPyC interface. This finding is in agreement with the SEM analysis discussed in Section 4.1.1.1, which indicates a maximum penetration of precipitates into the $\mathrm{SiC}$ layer of about $15 \mu \mathrm{m}$. The specimen from TEM Position $2 \mathrm{~b}$ was removed from the $\mathrm{SiC}$ layer immediately adjacent to and perpendicular to the IPyC$\mathrm{SiC}$ interface. Most of the precipitates found in the $\mathrm{SiC}$ layer at this location are identified as containing $\mathrm{Pd}$ and $\mathrm{U}$ as shown by the EDS spectrum in Figure 23. (The EDS spectra also show Si, but it is not possible to tell from the spectra whether the $\mathrm{Si}$ is from the matrix or the precipitate.) The precipitates in this region were identified during the preliminary SEM/EDS analysis as containing Pd.

The TEM specimen from Position 3 was taken perpendicular to and spanning the IPyC-SiC interface. The precipitates in this region were characterized as containing Pd and U using TEM-EDS. Selected images of precipitates on the $\mathrm{SiC}$ grain boundaries as well as in the IPyC from position 3 are shown in Figure 24. At this time, it is not possible to tell whether Ag is present because of peak pverlaps with $\mathrm{U}$ and $\mathrm{Pd}$. More detailed study of $\mathrm{Ag}$ in precipitates is planned for the future. 


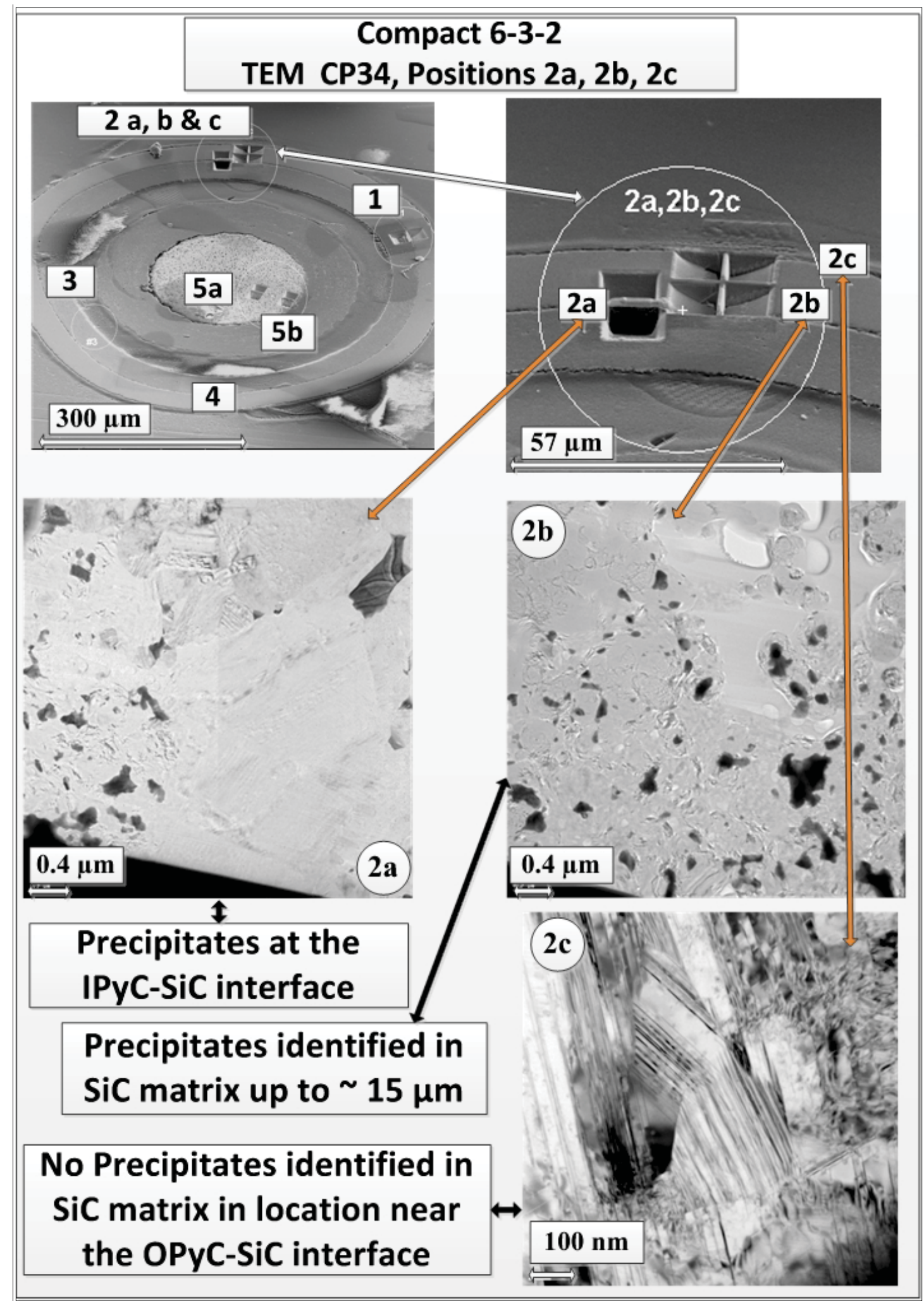

Figure 22. TEM micrographs for Specimens $2 a, 2 b$, and $2 c$ showing that precipitates are visible in Position $2 a$ and $2 b$, but no precipitates were observed at Position $2 c$. 


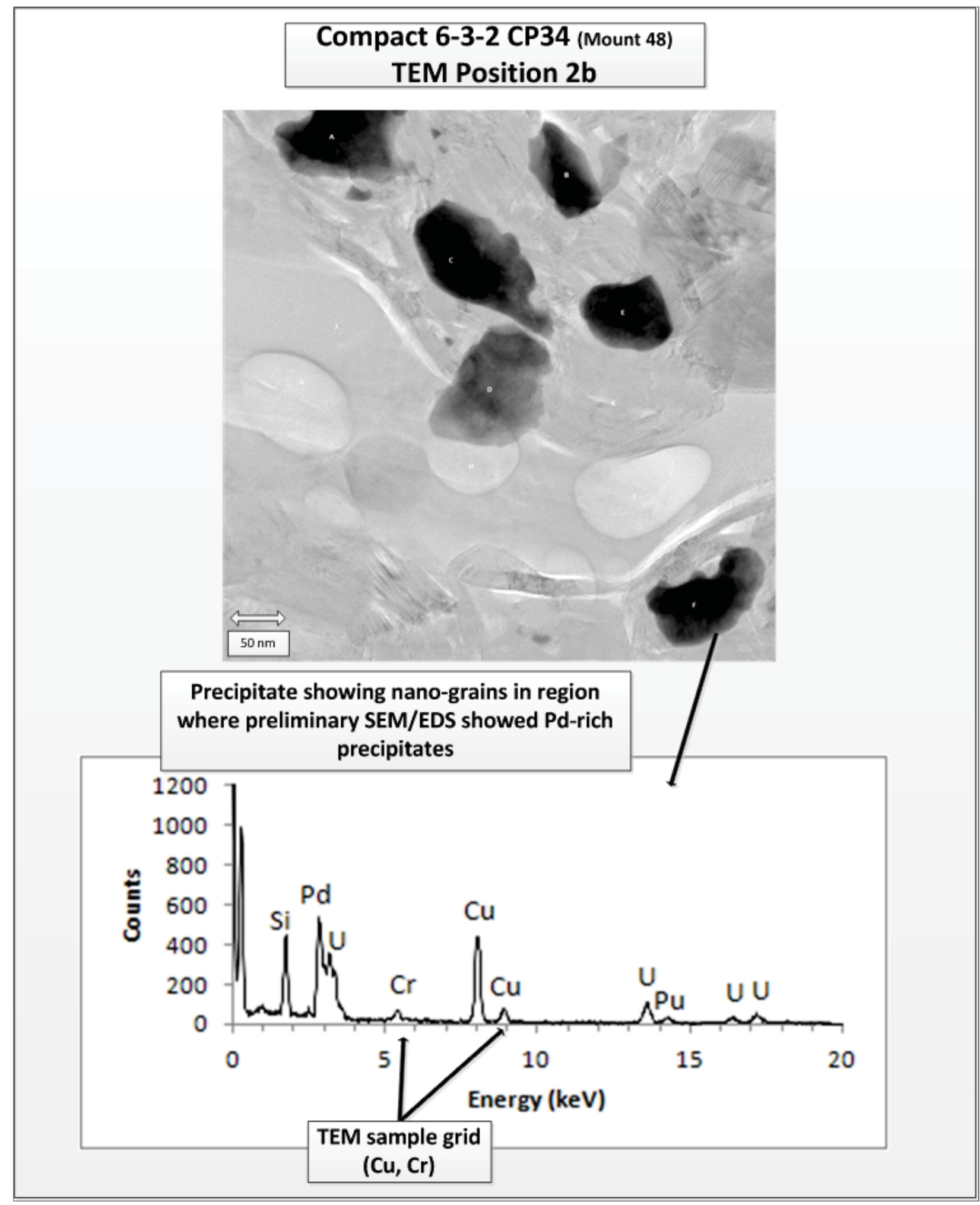

Figure 23. TEM micrograph showing representative images of precipitates in the SiC layer of CP34 from Compact 6-3-2, TEM Position 2b. 


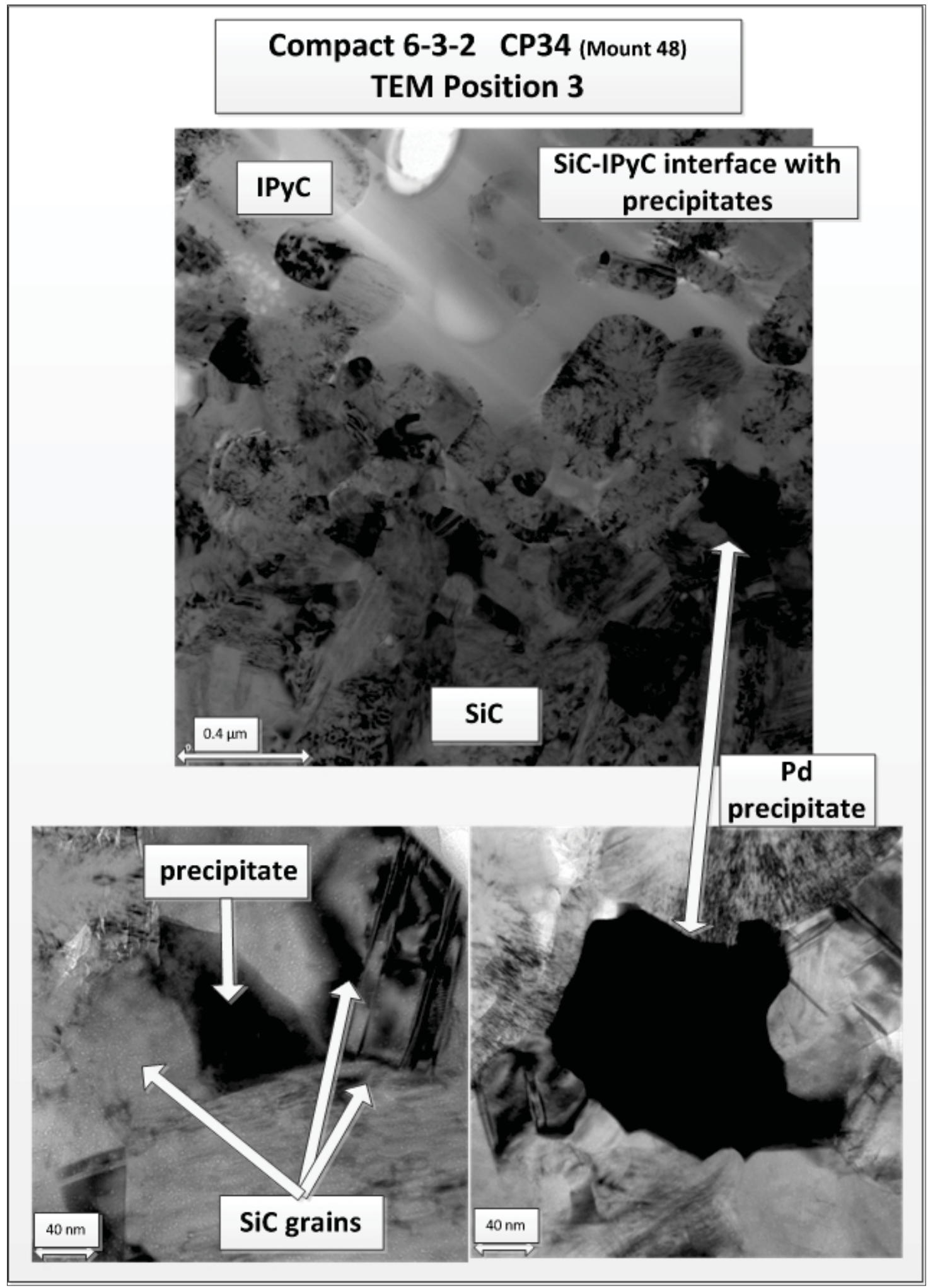

Figure 24. TEM micrograph showing selected images of precipitates on the SiC grain boundaries as well as in the IPyC of CP34 from Compact 6-3-2, TEM Position 3. 


\subsubsection{Precipitate Identification}

TEM data on precipitates were collected from CP 34 samples 1a, 1b, 2a, 2b and 3.. Precipitates in CP34 sample 1a were from a PyC layer. Precipitates in CP34 samples 1b, 2a, 2b, and 3 were in SiC.

In many cases, only TEM images, EDS spectra, or diffraction data were collected from each precipitate. Because the precipitates are small, tilting an individual precipitate to record diffraction patterns from different zone axes was difficult. Instead, precipitates in strongly diffracting orientations were identified in images and their diffraction patterns were recorded. It was therefore hypothesized that all of the remaining precipitates were from the same phase, subject to confirmation from detailed analysis of the data. No EDS analysis was completed for precipitates in the PyC layer in CP34 sample 1a. This analysis will be completed in the future.

Qualitative analyses of the EDS spectra showed significant concentrations of U, Pd, and Si, with some spectra also having significant concentrations of C and Zr. Semi-quantitative analyses of normalized atomic percentages of $\mathrm{Si}, \mathrm{Pd}$, and $\mathrm{U}$ (Table 3) showed similar compositions, with $\sim 40$ at $\% \mathrm{Si}, 45 \mathrm{at} \% \mathrm{Pd}$, and 10 to $15 \% \mathrm{U}$. This similarity in proportions of $\mathrm{U}, \mathrm{Pd}$, and Si suggested that these elements occurred in the same phase. Although concentrations of $\mathrm{Zr}$ were usually not quantified in most cases, inspection of the spectra suggested that they ranged from insignificant to a maximum of $\sim 32$ at $\%$ for the spectrum quantified in Table 3.

Table 3. TEM-EDS analyses from CP34, normalized so that the sum of the elements shown is $100 \%$.

\begin{tabular}{rlcccc}
\hline TEM & \multicolumn{1}{c}{ Figure ID in Original } & & & \\
Sample & Electron Microscopy Database & at $\%$ Si & at $\%$ Zr & at $\%$ Pd & at $\%$ U \\
\hline 1b & Pics 4, 5, and 6 & 24.34 & 32.49 & 21.57 & 21.60 \\
2a & Pics 1, 1a, 1b & 39.47 & N/A & 45.66 & 14.86 \\
& Pic 2, 2A & 40.72 & N/A & 43.79 & 15.49 \\
2b & Pic 2 Spot A & 40.43 & N/A & 47.45 & 12.12 \\
& Pic 2 Spot B & 42.66 & N/A & 45.48 & 11.85 \\
& Pic 2 Spot C & 38.92 & N/A & 47.26 & 13.82 \\
& Pic 2 Spot D & 40.81 & N/A & 46.55 & 12.64 \\
& Pic 2 Spot E & 49.78 & N/A & 38.95 & 11.27 \\
& Pic 2 Spot F & 43.37 & N/A & 44.36 & 12.27 \\
& Pic 5 & 40.18 & N/A & 45.86 & 13.96 \\
& Pic 7 Spot A & N/A & 44.16 & 15.18 \\
& Pic 19 & 40.66 & & 28.40 & 9.69 \\
\hline
\end{tabular}

Considerations for interpreting Table 3 include:

- Carbon is present in many of the spectra, but is not included because it is notoriously difficult to quantify. Because concentrations of all of the analyzed elements are normalized to a total of $100 \%$ in TEM data reduction, errors in the reported concentration of one element (such as C) will cause errors in concentrations of all of the others. If there are no peak overlaps between quantified and unquantified elements, concentrations can be renormalized to show only elements of interest without changing the relative proportions of these elements.

- Some or all of the $\mathrm{C}$ and $\mathrm{Si}$ may be from the matrix rather than the precipitates. $\mathrm{Zr}$ is present in many of the spectra (and significant in a few). However, these spectra were not quantified and are therefore not listed in Table 3. (Zr can be introduced as an artifact in EDS spectra collected with an objective aperture inserted, but doesn't appear in spectra without the aperture. Follow-up work needs to ensure that the aperture is removed before obtaining EDS spectra). 
- Relatively small (but still qualitatively significant) concentrations of other elements such as Pu were neglected in the analyses.

- Other elements such as Ag may be present, but cannot be recognized because of x-ray peak overlaps.

Phase identification was based on comparing the chemical and diffraction data to that in the International Centre for Diffraction Data's PDF4+ database, which contains over 300,000 records with detailed information that can be used to identify specific phases. Qualitative chemical analyses were used to reduce the number of possible identifications to materials containing some combination of $\mathrm{C}, \mathrm{Si}, \mathrm{Zr}$, $\mathrm{Pd}$, and $\mathrm{U}$. Elimination of all $\mathrm{SiC}$ structures except $3 \mathrm{C}, 4 \mathrm{H}$, and $6 \mathrm{H}$ reduced the number of possible entries to about 550. The International Centre for Diffraction Data's Sieve+ 2010 software was used to further limit the number of possible identifications. Still, about 125 possible records were consistent with the qualitative chemical analyses and matched some or all of the d-spacings in diffraction patterns.

As this number of records was too large for individual consideration to be practical, a different approach was chosen. Because the semi-quantitative interpretation of the EDS data suggested that many of these analyses represented the same phase, data analysis focused on finding a single phase that could produce all of the diffraction patterns from precipitates in $\mathrm{SiC}$ matrices. Nothing in the analysis precludes the possibility that there is more than one such phase. However, the likelihood of a unique identification increases with the number of different zone axes represented by the diffraction patterns.

All of the diffraction patterns from precipitates in the SiC layer in CP34 could be produced by single crystals of $\mathrm{UPd}_{2} \mathrm{Si}_{2}$ (PDF4+ card number 00-047-1029). Six zone axes were identified for precipitates found in CP34 as shown in Figures 25 and 26. Relative intensities of some reflections differed between diffraction patterns from different precipitates in the present data, as well as between different cards for $\mathrm{UPd}_{2} \mathrm{Si}_{2}$ in the PDF4+ database. These differences are not considered significant for phase identification. 


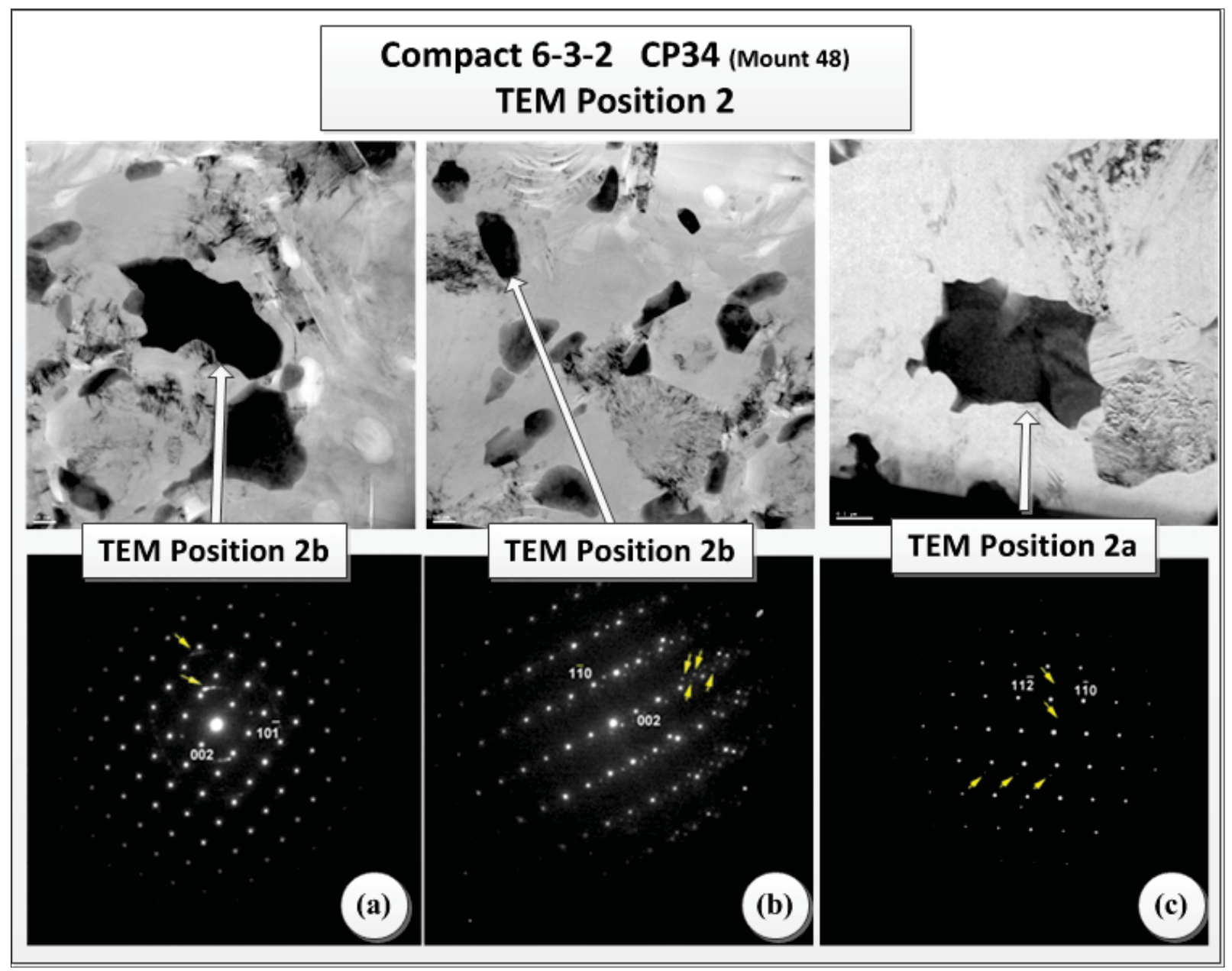

Figure 25. Zone-axis diffraction patterns from precipitates in CP34, indexed as $\mathrm{UPd}_{2} \mathrm{Si}_{2}$ showing a) zone [010] from CP 34 sample 2b picture 5, b) zone [110] from CP34 sample 2b, c) zone [111] from CP34 Sample 2a.

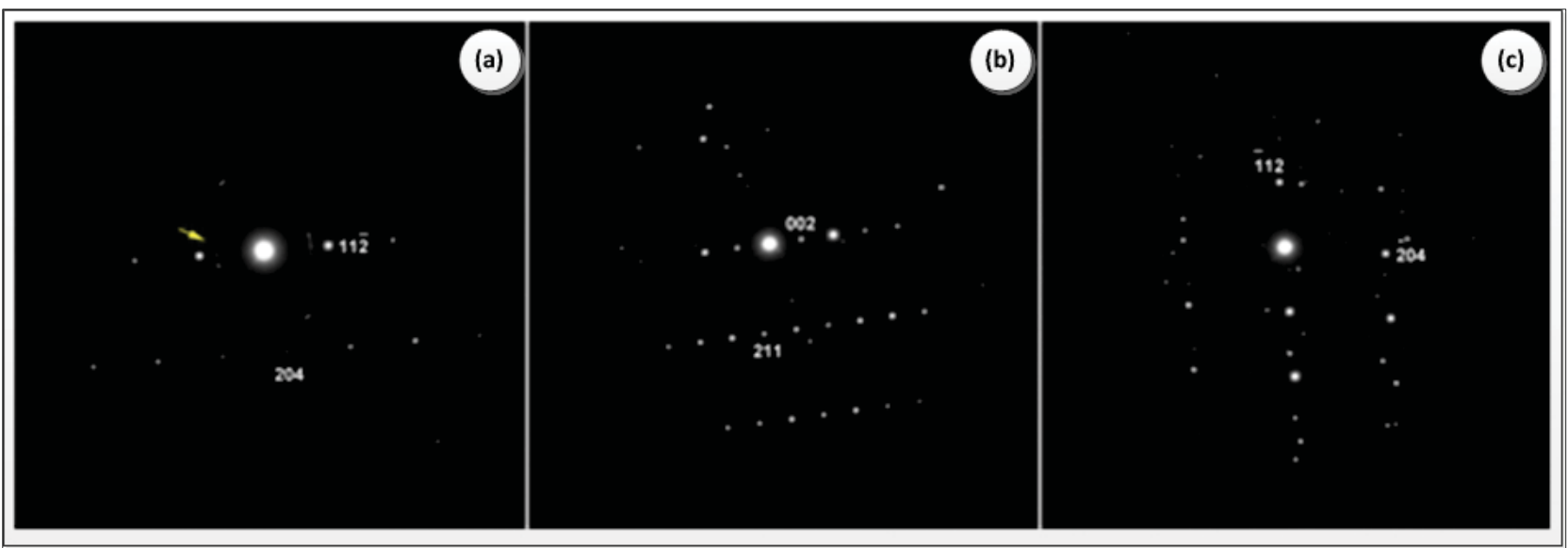

Figure 26. Three additional zone-axis diffraction patterns indexed as $\mathrm{UPd}_{2} \mathrm{Si}_{2}$ showing a) zone [2 -4 -1] from the same precipitate as part a) in Figure 29, with arrow showing a discontinuous ring that is not part of the single-crystal pattern. b) zone [1 -2 0]. c) zone [2 4 -1], considering bright reflections only. 
Proportions of $\mathrm{U}, \mathrm{Pd}$, and $\mathrm{Si}$ in the EDS data (Table 3) differ from those in stoichiometric $\mathrm{UPd}_{2} \mathrm{Si}_{2}$. These differences may be because of errors in the EDS quantification caused by incorrect deconvolution of peak overlaps between $\mathrm{U}, \mathrm{Pd}$, and $\mathrm{Si}$. However, it should be noted that all of the $\mathrm{UPd}_{2} \mathrm{Si}_{2}$ samples in the PDF4+ database are synthetic, and any information about the extent of any solid solutions has not been found.

Many of the diffraction patterns obtained by this study showed reflections that were not part of the $\mathrm{UPd}_{2} \mathrm{Si}_{2}$ single-crystal patterns. These extra reflections fell into three classes:

- Discontinuous rings (e.g., yellow arrows in Figure 25(a)), which have not yet been identified and may be from the matrix.

- Individual reflections that do not have a regular spatial relationship to the $\mathrm{UPd}_{2} \mathrm{Si}_{2}$ reflections are probably from the surrounding matrix material. Fainter, slightly streaked, reflections in one of the two diffraction patterns indexed as representing the [111] zone (e.g., yellow arrows in Figure 25(c)) may also be from the matrix, despite their regular spatial arrangement.

- Reflections with a regular spatial relationship to the $\mathrm{UPd}_{2} \mathrm{Si}_{2}$ reflections occurred in all of the [110] zone-axis patterns (e.g., yellow arrows in Figure 25(b)), in one of the [010] patterns, and in the only [2 4 -1] pattern recorded (Figure 26(c)). These reflections need to be investigated further because they may contain useful information about crystal structures or crystallographic relationships between the precipitates and the matrix.

The precipitates from which data was collected were chosen because they were in strongly diffracting orientations - many images are too dark to show internal details. Others show numerous dark areas $\sim 10 \mathrm{~nm}$ across as in Figure 27. It is not possible to determine from the present data whether these dark areas are inclusions (nanocrystals of a different phase from the surrounding material) or the same phase as the rest of the inclusions but appear darker because they are diffracting more strongly. It is also possible that the dark areas are in the matrix, but appear inside the precipitate because the matrix and precipitate overlap in the image. The sharp reflections in the diffraction patterns are not consistent with diffraction from nanocrystals (even if all of the nanocrystals have the same crystallographic orientation relative to the beam), leading to the inference that the majority of each precipitate is a single crystal producing the diffraction pattern. Further detailed analysis is required to understand the origins of both the dark spots in the precipitates and the origin of the extra reflections. 


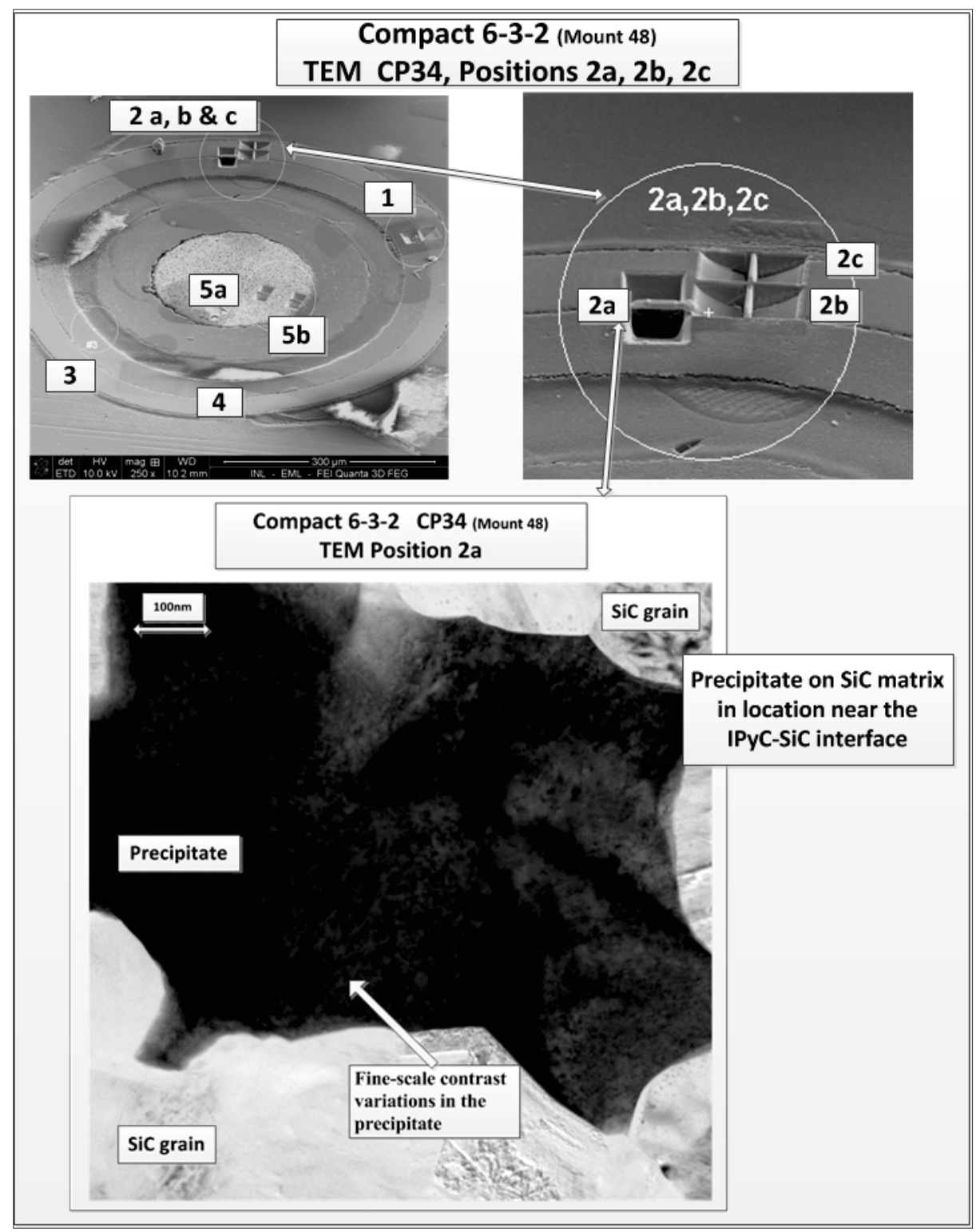

Figure 27. A precipitate surrounded by SiC; fine-scale contrast variations similar to those shown here are commonly observed in precipitates in AGR-1 SiCand IPyC matrices.

Further investigation is required to identify the phase containing $\mathrm{Zr}$, which is present in significant concentrations in some precipitates but absent in spectra from other precipitates. This variation in the concentration of $\mathrm{Zr}$ relative to those of $\mathrm{U}, \mathrm{Pd}$, and $\mathrm{Si}$ suggests that $\mathrm{Zr}$ is in a different phase and that EDS spectra with $\mathrm{Zr}$ represent mixtures of $\mathrm{UPd}_{2} \mathrm{Si}_{2}$ with a $\mathrm{Zr}$-bearing phase. EDS spectra from the matrix do not show $\mathrm{Zr}$, suggesting that it is associated with the precipitates. There are several hypotheses to explain where the $\mathrm{Zr}$ might be:

- $\quad \mathrm{The} \mathrm{Zr}$ is in solid solution in the $\mathrm{UPd}_{2} \mathrm{Si}_{2}$. (The wide variation in the proportions of $\mathrm{Zr}$ to $\mathrm{U}, \mathrm{Pd}$, and Si would require an extensive range of solid solution compositions. Although not ruled out at this stage, an extensive solid solution involving Zr seems unlikely.) 
- The $\mathrm{Zr}$ is a separate phase that occurs either in the small dark areas inside the precipitates or along their edges, and is not recognized in any of the $\mathrm{UPd}_{2} \mathrm{Si}_{2}$ diffraction patterns for one of the following reasons:

- Its reflections always overlap those of $\mathrm{UPd}_{2} \mathrm{Si}_{2}$. (This hypothesis seems highly unlikely since six $\mathrm{UPd}_{2} \mathrm{Si}_{2}$ zone axes were identified, and reflections from a high-Zr phase would have to overlap $\mathrm{UPd}_{2} \mathrm{Si}_{2}$ reflections in all of them.)

- It is nanocrystalline or amorphous, and therefore does not diffract strongly enough to be recognized as a separate phase. (In this context, it is important to measure the radii of rings such as those in Figures 25(a) and 26(a), to determine whether they could be produced by the matrix or might be from a nanocrystalline high-Zr phase.)

- Zr can also appear as an artifact in EDS spectra collected with the objective aperture inserted. However, this does not explain all of the $\mathrm{Zr}$, as it also appears in some spectra collected with the objective aperture fully retracted.

Although no conclusive evidence is found for the presence of Ag from the current EDS data, it may be useful to speculate about whether it could be incorporated into $\mathrm{UPd}_{2} \mathrm{Si}_{2}$. A number of ternary silicides with $\mathrm{ThCu}_{2} \mathrm{Si}_{2}$-type structure (body-centered tetragonal, space group I4/mmm) have been synthesized. ${ }^{6,7,8,9,10}$ These silicides have the general formula $\mathrm{RX}_{2} \mathrm{Si}_{2}$, where $\mathrm{R}$ is $\mathrm{U}$, Th, or Gd and $\mathrm{X}$ is a 4d transition metal such as $\mathrm{Cr}, \mathrm{Mn}, \mathrm{Fe}, \mathrm{Co}, \mathrm{Ni}, \mathrm{Cu}, \mathrm{Ru}, \mathrm{Rh}, \mathrm{Pd}, \mathrm{Ir}, \mathrm{Pt}$, or $\mathrm{Au}$. Specific examples include $\mathrm{UPd}_{2} \mathrm{Si}_{2}$ and $\mathrm{GdAg}_{2} \mathrm{Si}_{2}$. Although we are not aware of any reported syntheses of $\mathrm{UAg}_{2} \mathrm{Si}_{2}$, it seems plausible that it would also have a $\mathrm{ThCu}_{2} \mathrm{Si}_{2}$-type structure. Since $\mathrm{Ag}$ and $\mathrm{Pd}$ have identical atomic radii (both $0.144 \mathrm{~nm}$ for the pure elements ${ }^{11}$, it seems reasonable to speculate that any $\mathrm{Ag}$ atoms that might be present would substitute for $\mathrm{Pd}$ atoms in a $\mathrm{U}(\mathrm{Ag}, \mathrm{Pd})_{2} \mathrm{Si}_{2}$ solid solution rather than forming a separate phase. (Even though $\mathrm{Zr}$ is a $4 \mathrm{~d}$ transition metal, its atomic radius is significantly different from that of $\mathrm{Pd}$, and an extensive $\mathrm{U}(\mathrm{Zr}, \mathrm{Pd})_{2} \mathrm{Si}_{2}$ solid solution seems unlikely.)

$\mathrm{U}$ and $\mathrm{Pu}$ often substitute for one another in metals and intermetallics, and it seems reasonable to speculate that low concentrations of $\mathrm{Pu}$ could substitute for $\mathrm{U}$ in $\mathrm{UPd}_{2} \mathrm{Si}_{2}$.

\subsubsection{TEM-EDS identification of $\mathrm{Sr}$ and $\mathrm{Pu}$}

All of the EDS spectra of U-Pd-Si precipitates from the SiC layer in CP34 and CP35 that were recorded in graphical format (those saved as TIFF or EMSA files) show a small peak at approximately $14.2 \mathrm{keV}$ as shown in Figure 28. The ISIS software's automatic peak identification routine interprets this peak as $\mathrm{Sr} \mathrm{K} \alpha$, which has an energy of $14.163 \mathrm{keV}$; however, this energy would also be consistent with $\mathrm{Pu} \mathrm{L} \alpha 1(14.275 \mathrm{keV})$. In principle, determining whether this peak is $\mathrm{Sr}$ or Pu can be done by identifying other peaks from the same element (e.g., $\mathrm{Sr} L \alpha, \operatorname{Sr} \mathrm{L} \beta, \mathrm{Sr} \mathrm{K} \beta, \mathrm{Pu} \mathrm{M} \alpha, \mathrm{Pu} \mathrm{M} \beta$, or Pu L $\beta 1$ ). In practice, distinguishing between $\mathrm{Pu}$ and $\mathrm{Sr}$ is difficult because of a complicated series of peak overlaps that also involve $\mathrm{Si}, \mathrm{Zr}$, and U. Table 4 and Figure 29 show the relevant peak overlaps.

All the other $\mathrm{Sr}$ and $\mathrm{Pu}$ peaks except for $\mathrm{Pu} \mathrm{L} \beta 1$ are overlapped by high-relative-intensity peaks from other elements likely to be present. The Pu L $\beta 1$ peak is overlapped by the U L $\beta 9$-M5 peak, which has a very low relative intensity but might still appear in spectra with large numbers of counts that were collected from areas with high concentrations of U. Figure 30 shows a spectrum that has an unusually large number of counts in the U L-series peaks but does not have a peak at $\sim 18.3 \mathrm{keV}$. This spectrum demonstrates that $U$ should not be expected to produce a recognizable U L $\beta 9$-M5 peak, particularly in spectra with smaller numbers of counts. Since the peak at $\sim 18.3 \mathrm{keV}$ is not U $\beta$ B9-M5, it must be Pu L $\beta 1$. Once the Pu L $\beta 1$ peak it identified, it follows that the peak at $\sim 14.2 \mathrm{keV}$ is $\mathrm{Pu} \mathrm{L} \alpha 1$. 


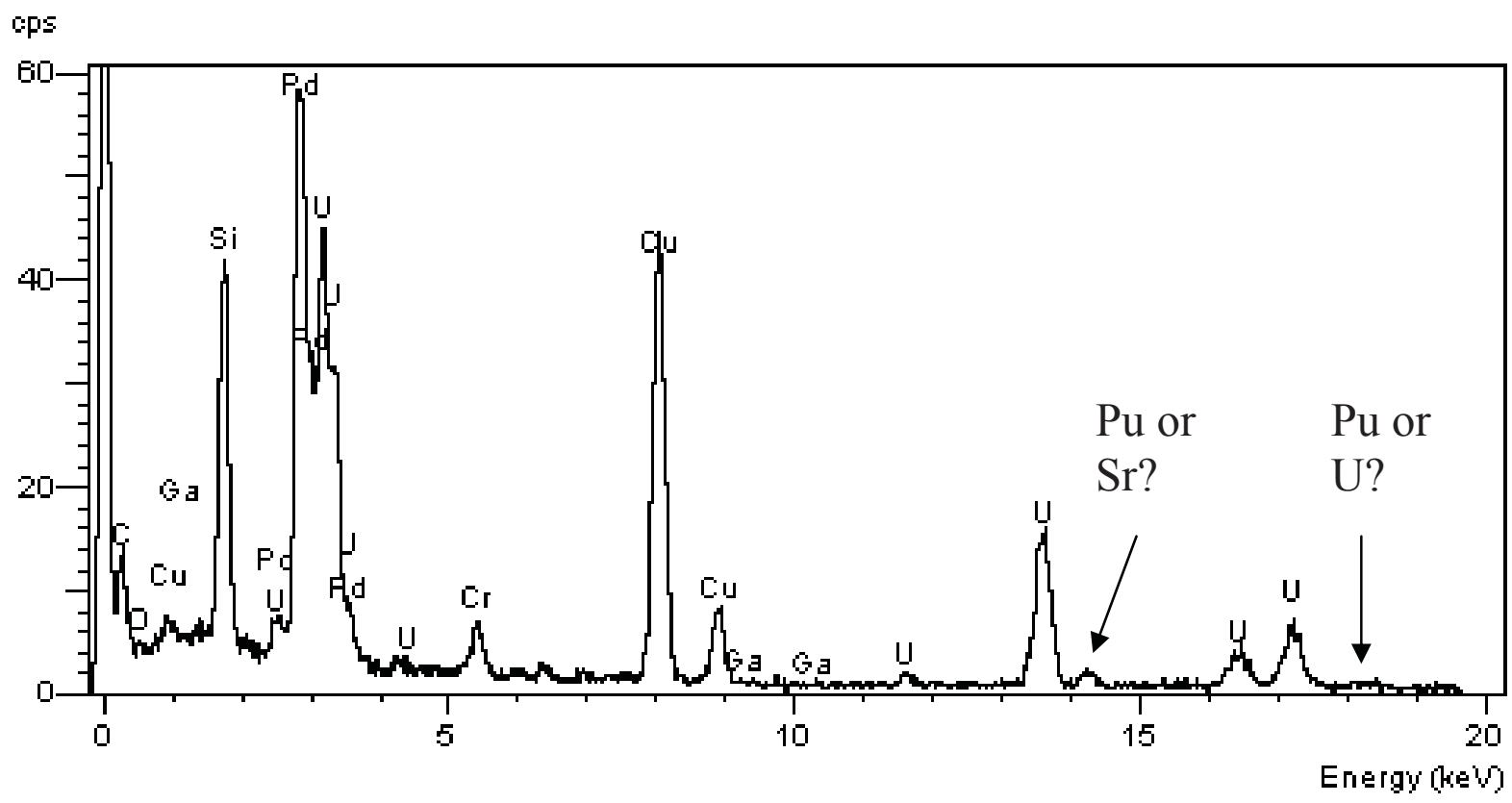

Figure 28. EDS spectrum showing small peak at $\sim 14.2 \mathrm{keV}$ that could be $\mathrm{Pu}$ or $\mathrm{Sr}$ and small peak at $\sim 18.3 \mathrm{keV}$ that could be Pu or U (original EML database identification: CP34 Sample 3, Picture 7, Spot A).

Table 4. Relevant $\mathrm{x}$-rays for distinguishing between low concentrations of $\mathrm{Sr}$ and $\mathrm{Pu}$, showing peak overlaps in spectra with much higher concentrations of $\mathrm{Si}, \mathrm{U}, \mathrm{Pd}$, and possibly some $\mathrm{Zr}$. 4,5

\begin{tabular}{|ccc|}
\hline x-ray & Energy (keV) & Overlapped by \\
\hline Sr L $\alpha$ & 1.806 & Si K $\alpha$ \\
Sr L $\beta$ & 1.871 & Si K $\alpha$ \\
$\mathrm{Pu} \mathrm{M} \alpha$ & 3.348 & $\mathrm{U} \mathrm{M} \beta$ \\
$\mathrm{Pu} \mathrm{M} \beta$ & 3.531 & $\mathrm{U} \mathrm{M} \gamma$ \\
$\mathrm{Sr} \mathrm{K} \alpha$ & 14.163 & $\mathrm{Pu} \mathrm{L} \alpha$ \\
$\mathrm{Pu} \mathrm{L} \alpha 1$ & 14.275 & $\mathrm{Sr} \mathrm{K} \alpha$ \\
$\mathrm{Sr} \mathrm{K} \beta$ & 15.837 & $\mathrm{Zr} \mathrm{L} \alpha$ \\
$\mathrm{Pu} \mathrm{L} \beta 1$ & 18.276 & $\mathrm{U} \mathrm{L} \beta 9-\mathrm{M} 5$ \\
\hline
\end{tabular}




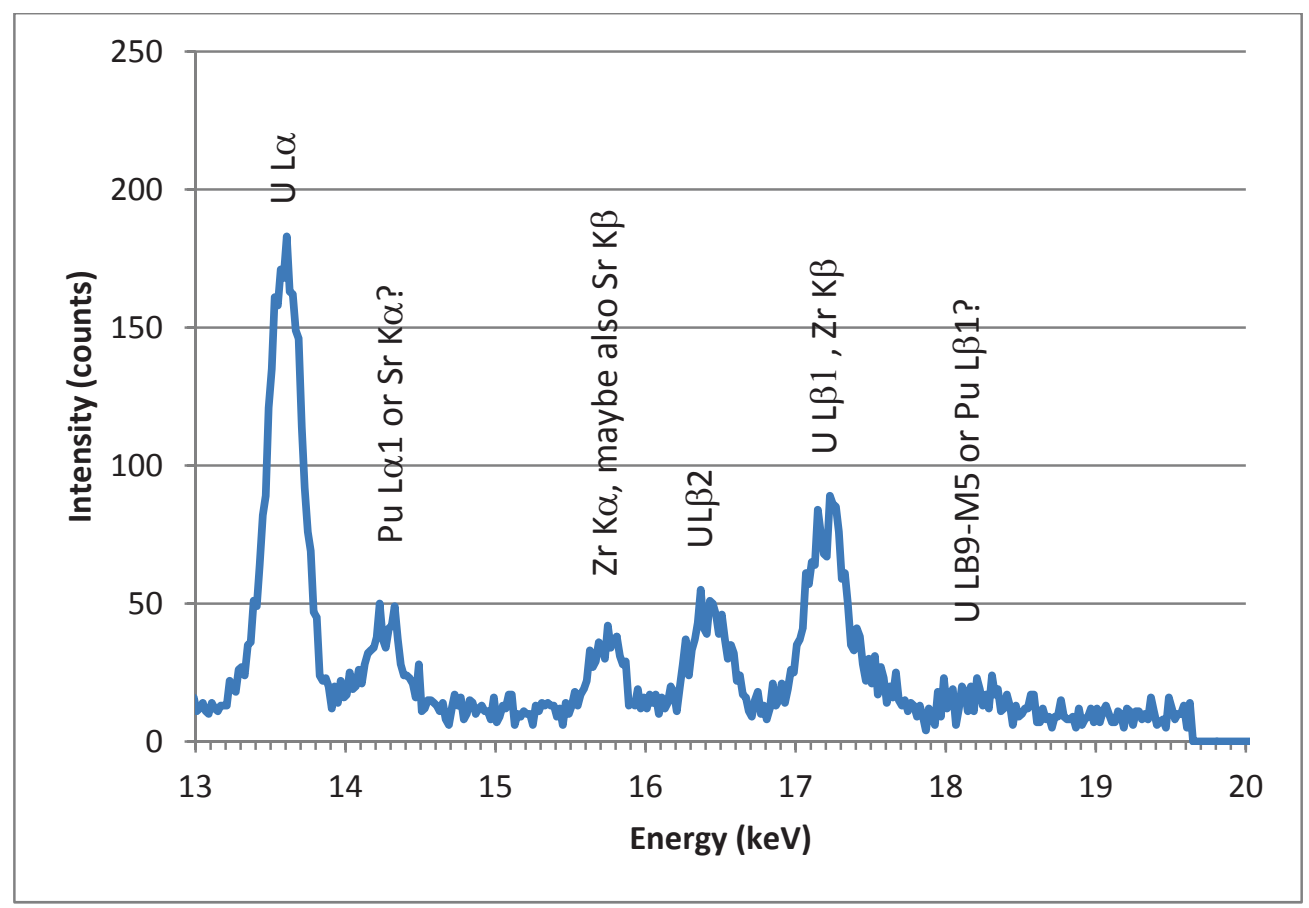

Figure 29. Expanded section of EDS spectrum showing details of overlaps between the Sr K-series peaks, Pu L-series peaks, and peaks from $U$ and $\mathrm{Zr}$ (Original EML database identification: CP34 sample 2B Picture 2, Spot B). See Table 4 for Pu and Sr x-ray energies.

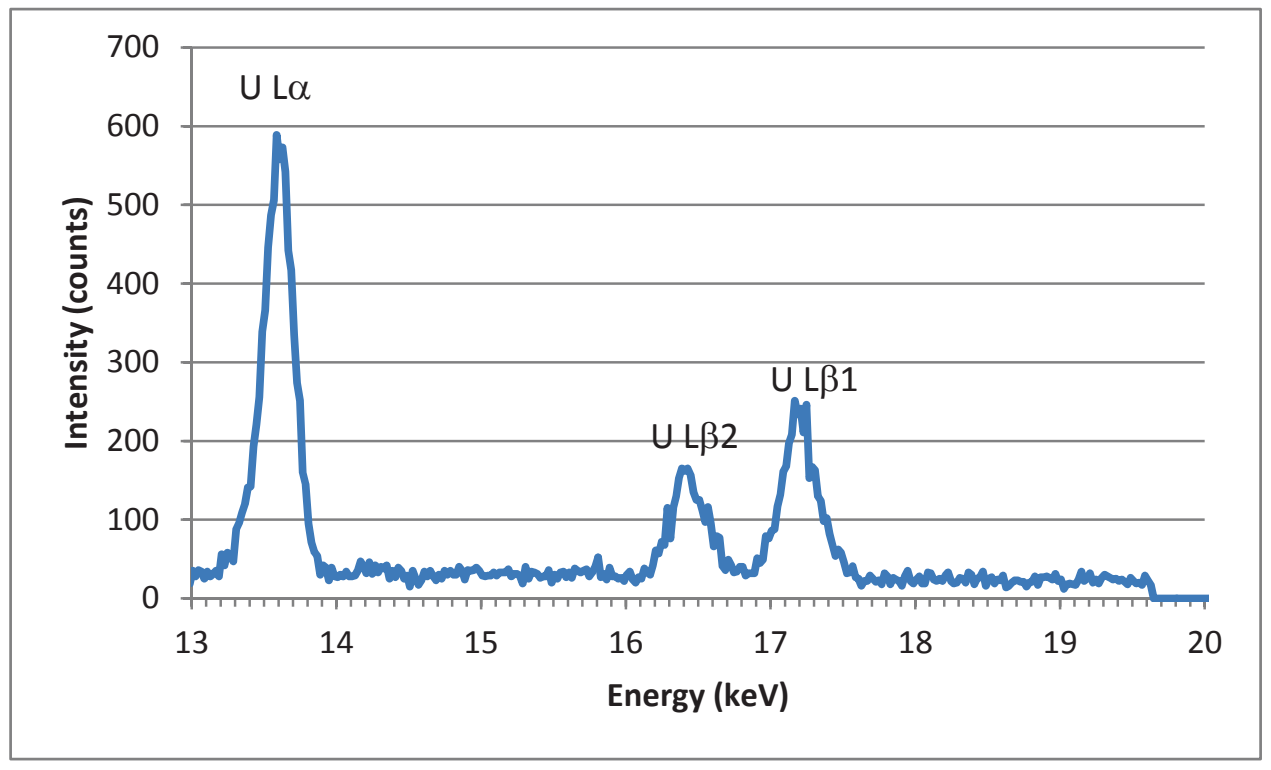

Figure 30. Expanded section of an EDS spectrum showing only peaks from U. Note that there is no U L $\beta 9$-M5 peak, even though the intensity in this spectrum is greater than that in Figure 29. (Original EML database identification: CP30 Picture 13). 


\subsubsection{Irradiation Effects on SiC and IPyC Microstructures.}

The irradiation effects on the $\mathrm{SiC}$ and IPyC microstructures are only briefly discussed in this report. A very detailed investigation and quantification of the loop vacancy, gas bubbles and cavities (voids) still needs to be completed. A funding proposal is being prepared to continue that work.

\subsubsection{SiC Layer}

No evidence was found of any phase transformation from the cubic to the hexagonal phase because of the irradiation cycle as determined by selected area diffraction (SAD) patterns measured on the different TEM samples prepared from CP34. Examples of representative SAD patterns are presented in Figure 31.

Selected area diffraction patterns were used to find the $\mathrm{SiC}$ lattice parameter $\left(\mathrm{a}_{\mathrm{o}}\right)$. Zone axis patterns were obtained and identified from the $\mathrm{SiC}$ layer. Using the d-spacing of standard single crystal of Alloy 6061 (> 96wt\% Al) the d-spacing of known SiC diffraction spots were obtained from each zone axis pattern. The d-spacings from various diffraction spots were used to find the $\mathrm{SiC}$ lattice parameter. The lattice parameters from an individual zone axis pattern were averaged to provide the $\mathrm{SiC}$ lattice parameter as shown in Figure 31.

The typical effects of irradiation on the SiC microstructure are shown in Figures 32 and 33, which are representative of two opposite parts of the $\mathrm{SiC}$ layer cross section. These figures also represents the irradiated microstructure at the SiC-IPyC interlayer (Figure 32(a), (b), (c), and Figure 33(c) and (d)) as well as on the outer edge of the $\mathrm{SiC}$ layer near the OPyC layer (Figure 33 (a), (b)). Although a full investigation to fully determine the loop and dislocation densities of this material was not completed, no significant difference between the inner and outer portions of the $\mathrm{SiC}$ was observed. Also, no denuded zone for dislocation loops along grain boundaries were observed during this preliminary study, but more detailed analysis will be done during follow-up analysis. Kondo et al. ${ }^{12}$ typically found denuded zones at grain boundaries in some areas for irradiated $\mathrm{SiC}$ at $1130^{\circ} \mathrm{C}$. This may be of importance for understanding the fission product transport mechanisms and will be investigated specifically in the areas where fission product precipitates were found. Visible loop density variations are however noted within the same sample at different locations as shown in Figure 33(a) and (b). At this point no conclusion can be reached from this observation and the results will require further analysis. The cavities were predominantly found to be spherical in shape which corresponds with the findings of Kondo et al. ${ }^{12}$ Future work will include an examination of the size, density and shape of cavities, as well as the size and density of the loops. 


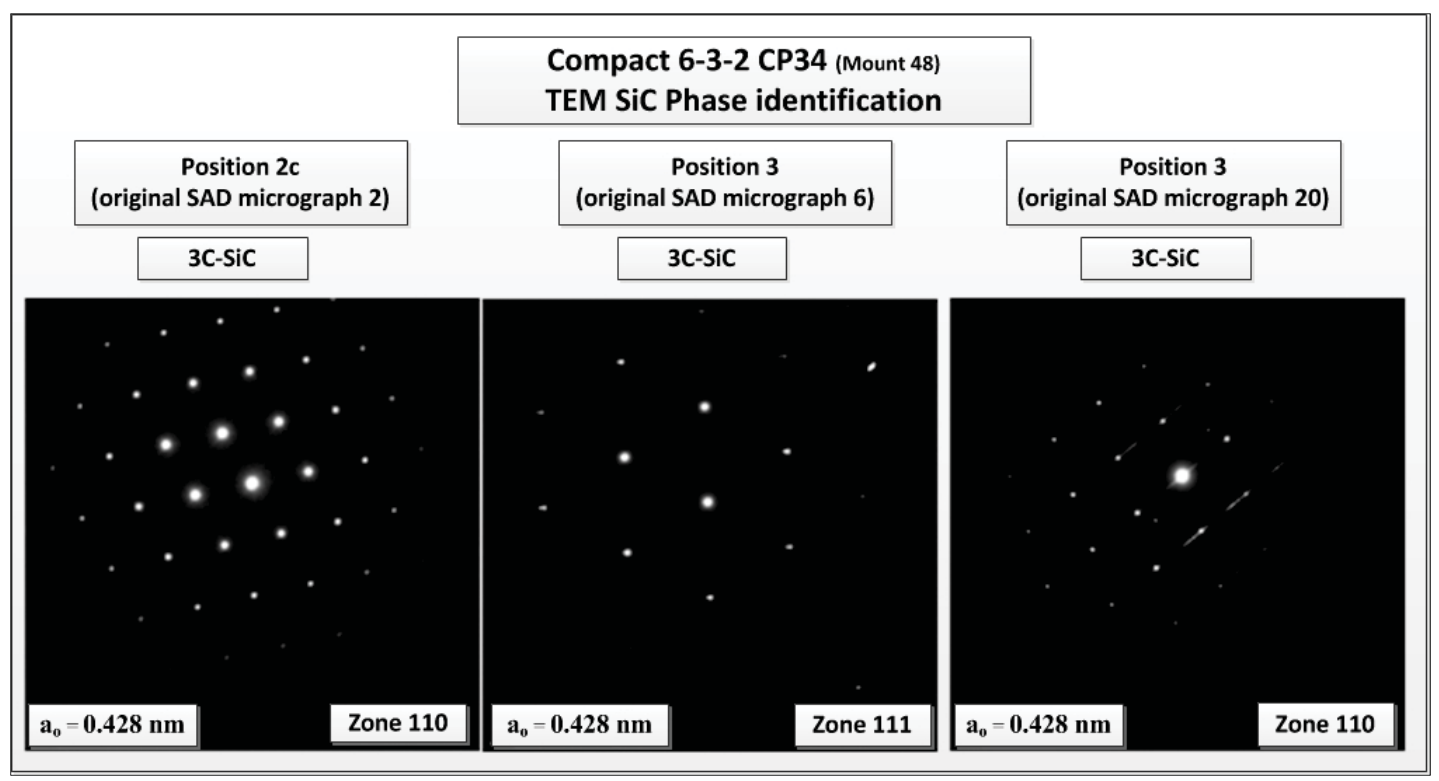

Figure 31. Summary of representative selected area diffraction patterns of the SiC layer of CP34 determined at different positions.

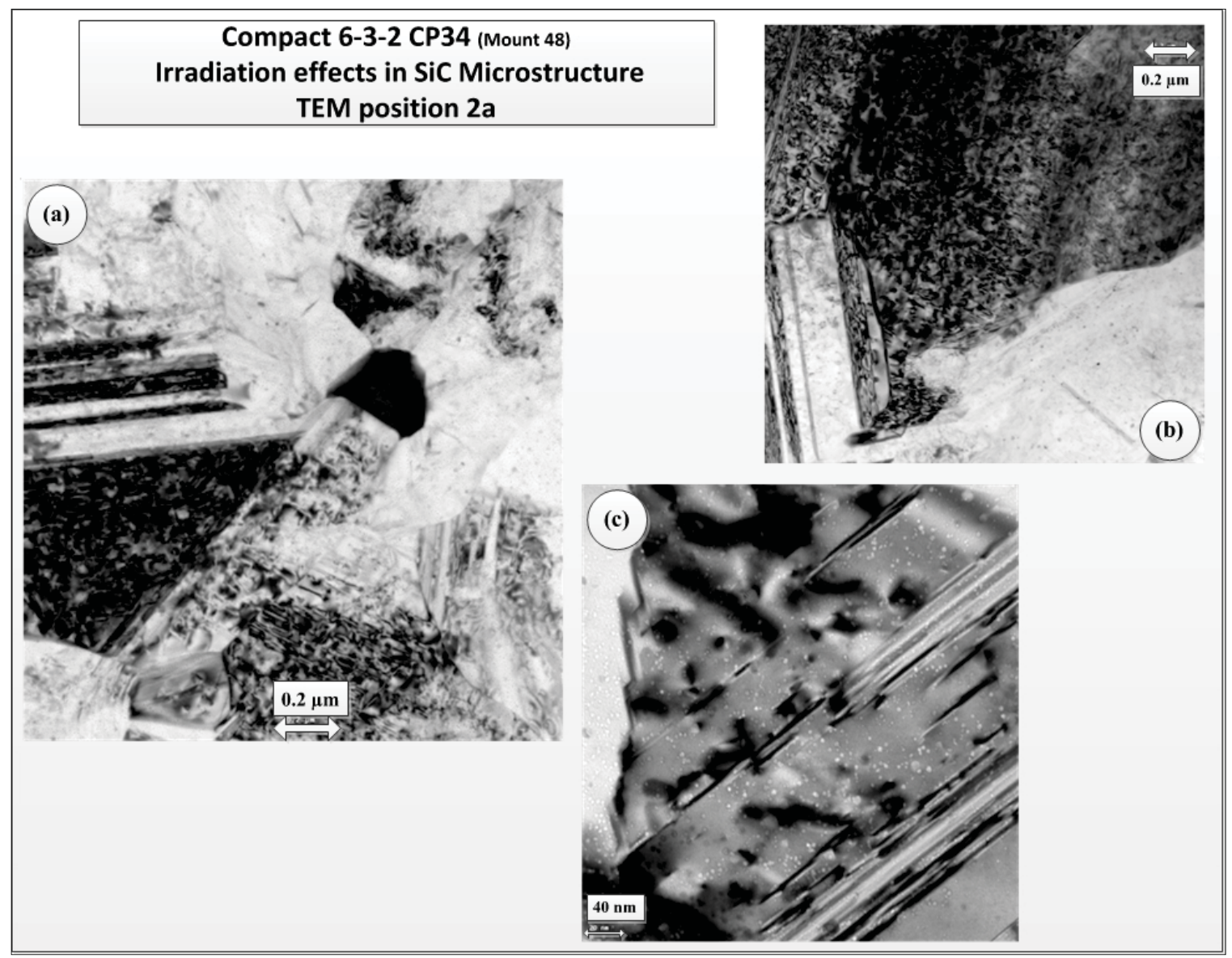

Figure 32. Bright Field TEM images showing irradiated microstructure of CP34 Position 2a, with (a) and (b) showing the typical dark spot defects and (c) showing the cavities caused by irradiation. 


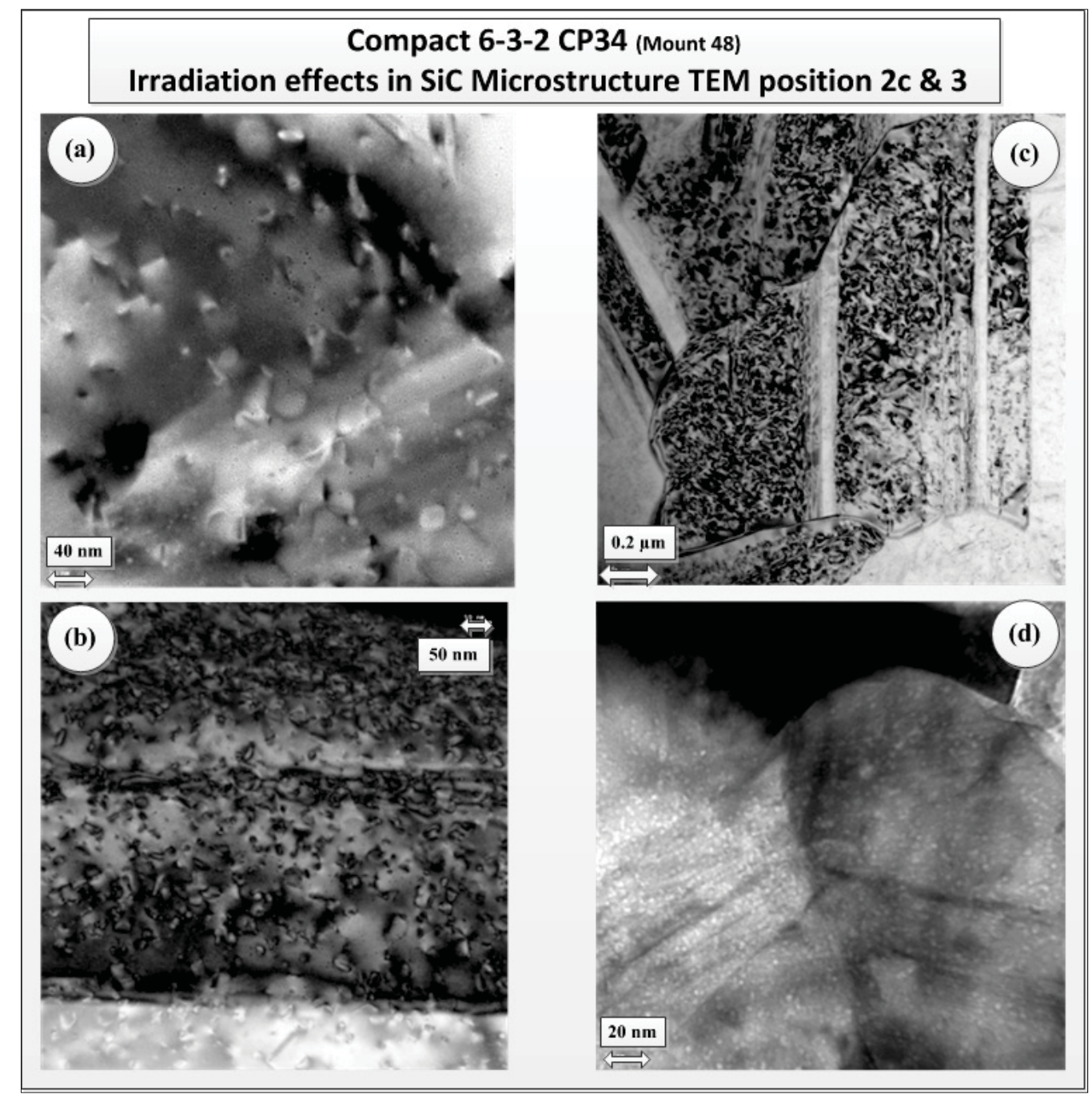

Figure 33. Bright field TEM images of the irradiated SiC microstructure of CP34 showing (a) and (b) variations in loop density at Positions $2 \mathrm{c}$ and (c) at Position 3, and (d) shows spherical cavities at Position 3.

\subsubsection{IPyC}

Very preliminary TEM observations of the IPyC layer in unirradiated AGR-1 coated particles showed randomly oriented crystallites 5-10 $\mathrm{nm}$ across (Figure 34), becoming less random near the interface with the $\mathrm{SiC}$. The corresponding selected area diffraction pattern for the unirradiated fuel is shown in Figure 34. Although more detailed work is in process to fully quantify the isotropic nature of the IPyC as a function of the four aperture diameters, some preliminary observations were made. Diffraction patterns from both the unirradiated and irradiated AGR-1 Baseline IPyC showed four rings whose radii correspond to d-spacings of approximately $3.45,2.08,1.69$, and $1.21 \AA$. Variations in intensity around the rings in the experimental diffraction patterns indicate preferred orientation. Diffraction patterns taken with the smallest available selected-area aperture (effective diameter $\sim 160 \mathrm{~nm}$ ), commonly show large variations in intensity, indicating a high degree of preferred orientation. These variations become "averaged out" in diffraction patterns with the largest aperture (effective diameter $\sim 1200 \mathrm{~nm}$ ). 
The basic TEM evaluation of the irradiated PyC in CP34 was mainly conducted on the IPyC near the $\mathrm{SiC}-\mathrm{IPyC}$ interface and a representative PyC microstructure is shown in Figure 35 with three selected area diffraction patterns obtained on three different areas shown in Figure 36.

Preliminary evaluation on the irradiated IPyC shows variation in intensity going around each ring. Although intensity variations within individual rings in the IPyC diffraction patterns after irradiation are observed for CP34 (Figures 36 (a), (b) and (c)) and are an indication that the IPyC is becoming anisotropic, no final conclusion can be made as a more detailed analysis is needed. For example, SAD patterns needs to be measured as a function of distance from the IPyC-SiC interlayer and aperture diameters. At this point, it can't be concluded whether temperature or neutron fluence is the predominant reason for the observed changes as more comparative studies need to be completed with other irradiated and unirradiated AGR-1 compacts as well as a comparison with data published in the literature. Previous work by Van Rooyen et al. ${ }^{13}$ however implies that it may be predominantly because of the temperature effect. More information and examination are needed to determine whether these differences are significant with comparative aperture sizes.

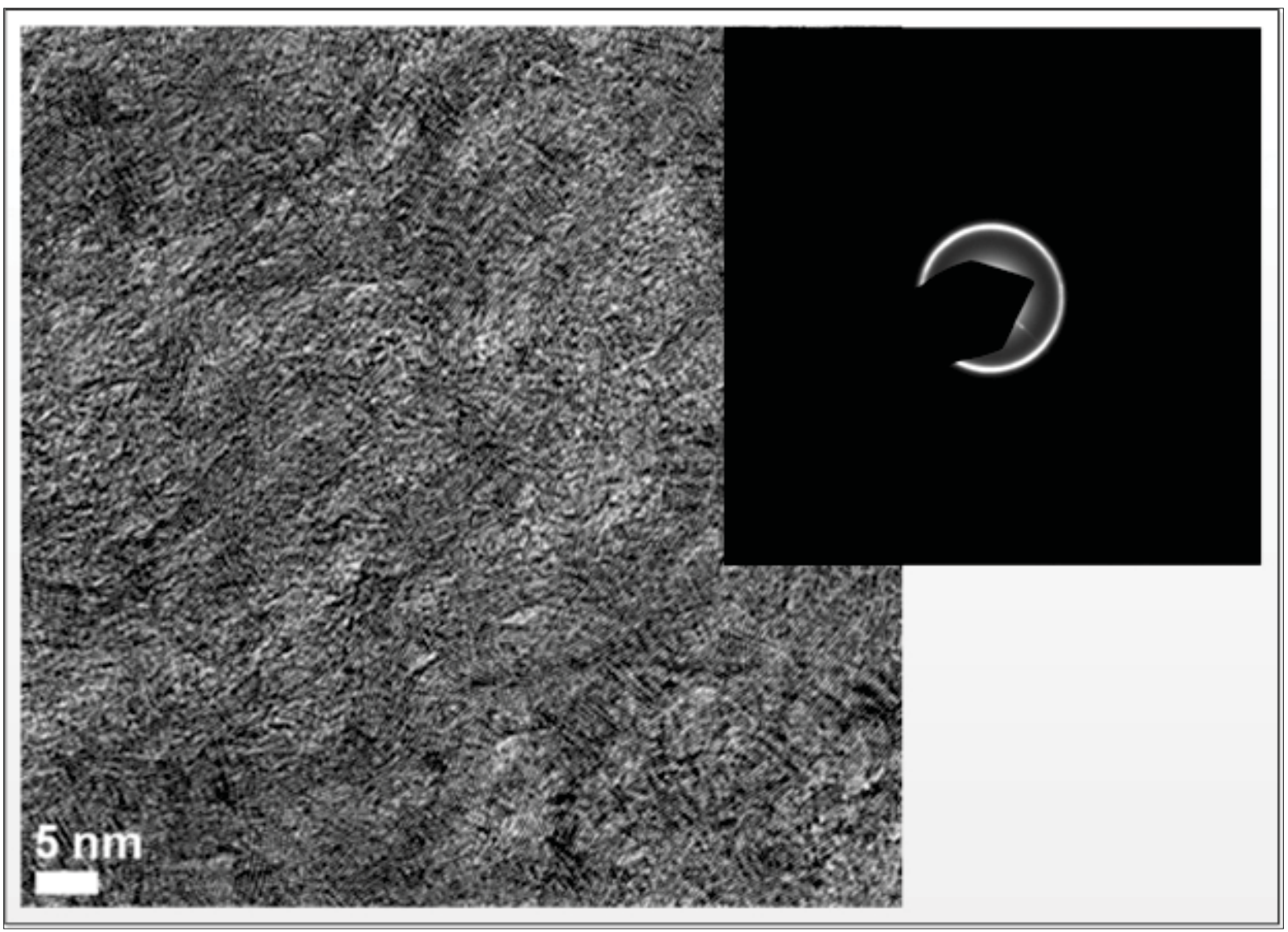

Figure 34: Image of unirradiated IPyC showing randomly oriented crystals 5-10 nm across with corresponding SAD pattern. 


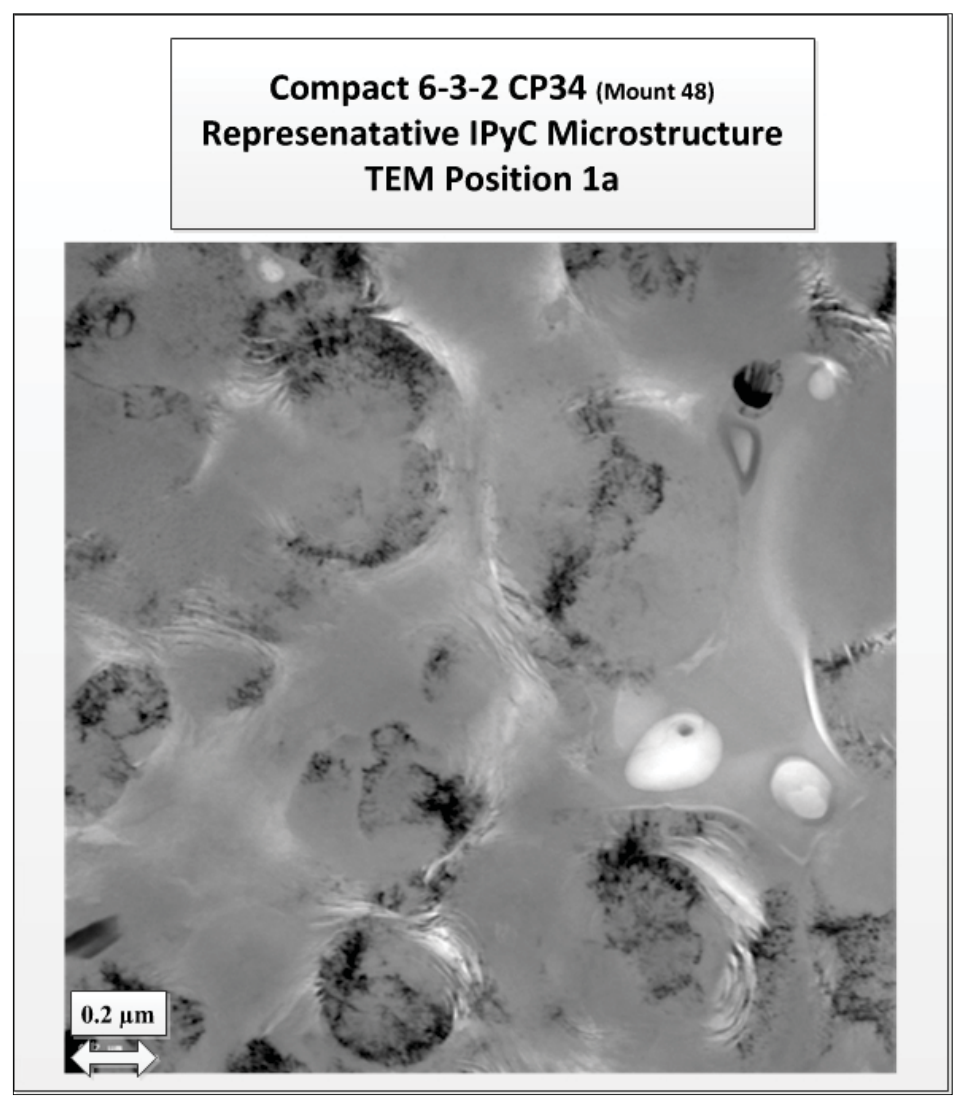

Figure 35. Bright field TEM image showing the typical IPyC microstructure of CP34.

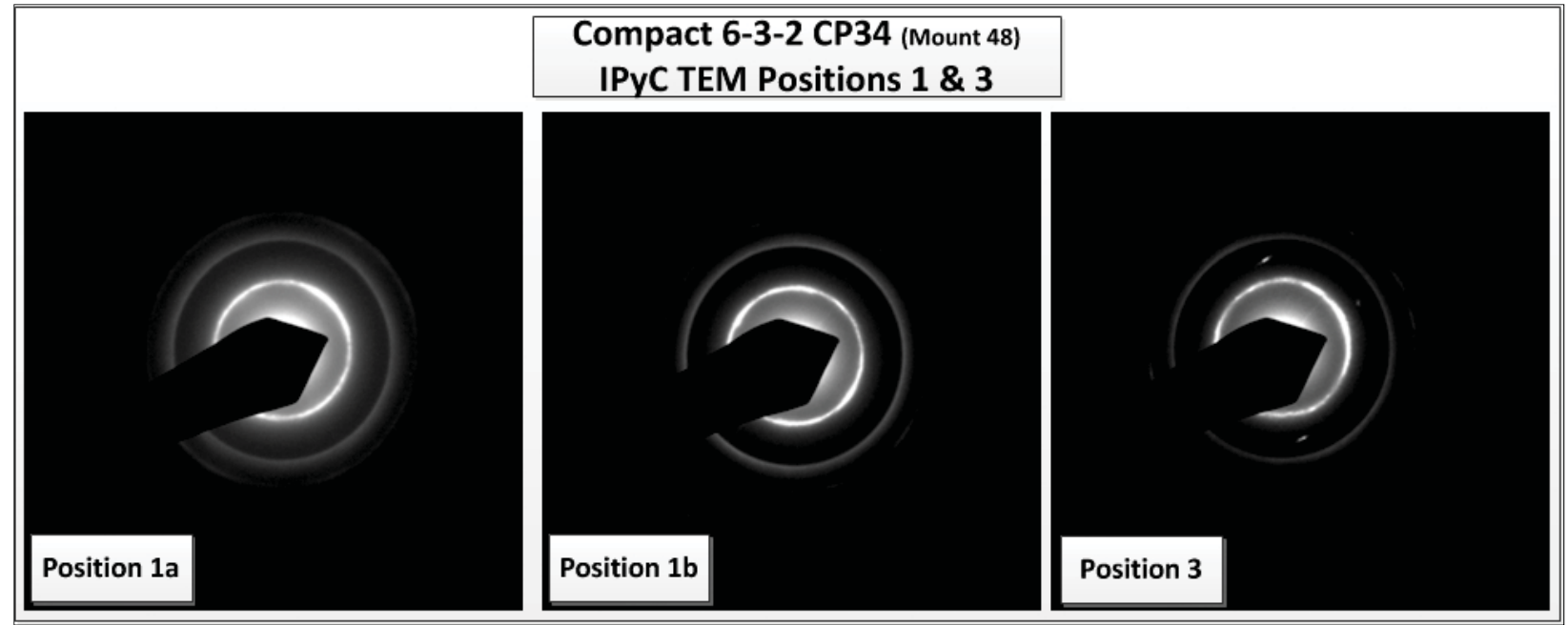

Figure 36. Selected area diffraction patterns as examples of preliminary data on IPyC structure of (a) CP34 Position 1a, (b) CP34 Position 1b, and (c) CP34 Position 3 (aperture sizes were not recorded during time of analysis and may not be the same for all the diffraction patterns). 


\subsubsection{IPyC-SiC Interface Integrity}

No evidence of IPyC-SiC interface debonding was observed in CP34 in all of the TEM samples (Positions 1, 2a, and 3) investigated. A typical SiC-IPyC interface is shown in Figure 37.

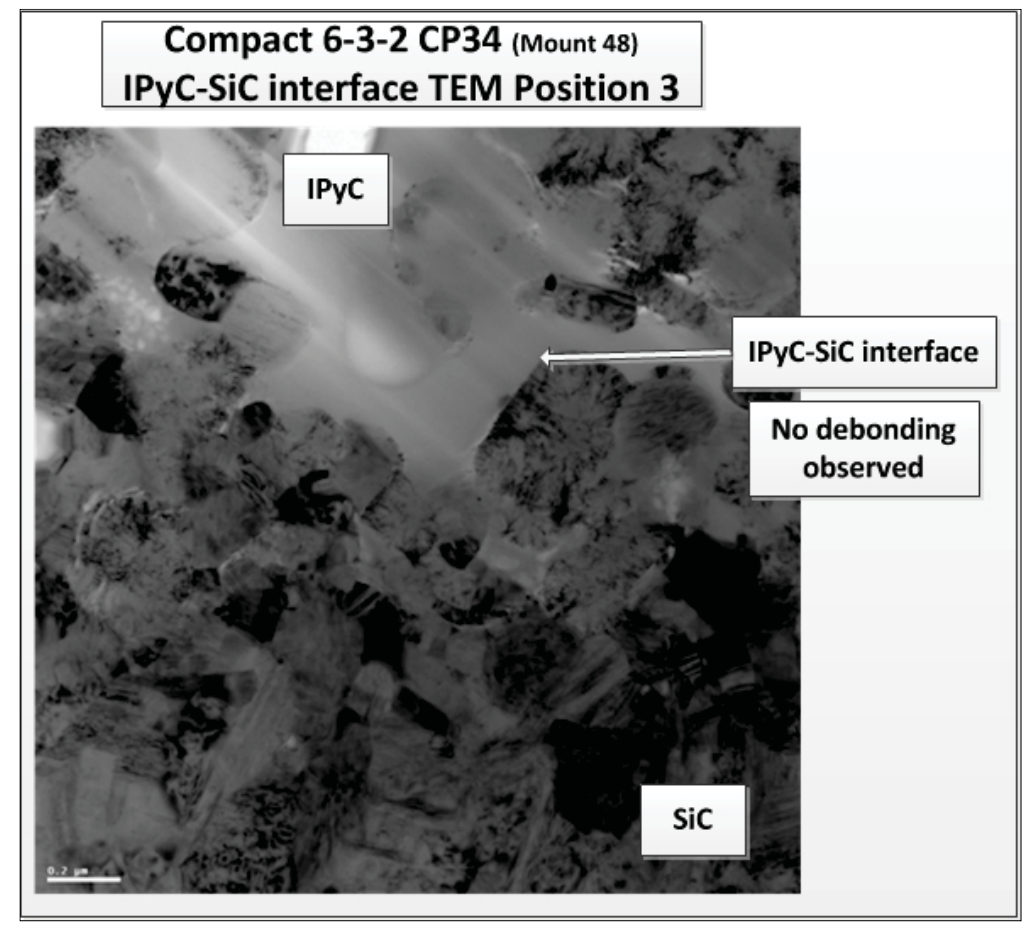

Figure 37. Bright field TEM image shows no evidence of SiC-IPyC interface debonding due to irradiation in CP34.

\section{$4.2 \quad \mathrm{CP} 35$}

\subsubsection{SEM Examination}

The same approach for the SEM analysis of CP34 was followed for CP35: a basic examination was performed that identified areas of precipitate clusters, which were then identified for further FIB preparation for TEM investigations. No EDS or WDS analysis was done during the SEM analysis. Figure 38 shows secondary electron images of CP35 with an inset at the upper right of an optical micrograph. No macroscopic cracks were observed in the $\mathrm{SiC}$ layer, but fissures are observed in the buffer layer. Figure 39 presents SEM micrographs of CP35 showing precipitates in the buffer, IPyC layer, and at the SiC-IPyC interlayer. Precipitates are not as clearly visible on the circumference compared with those of CP34. 


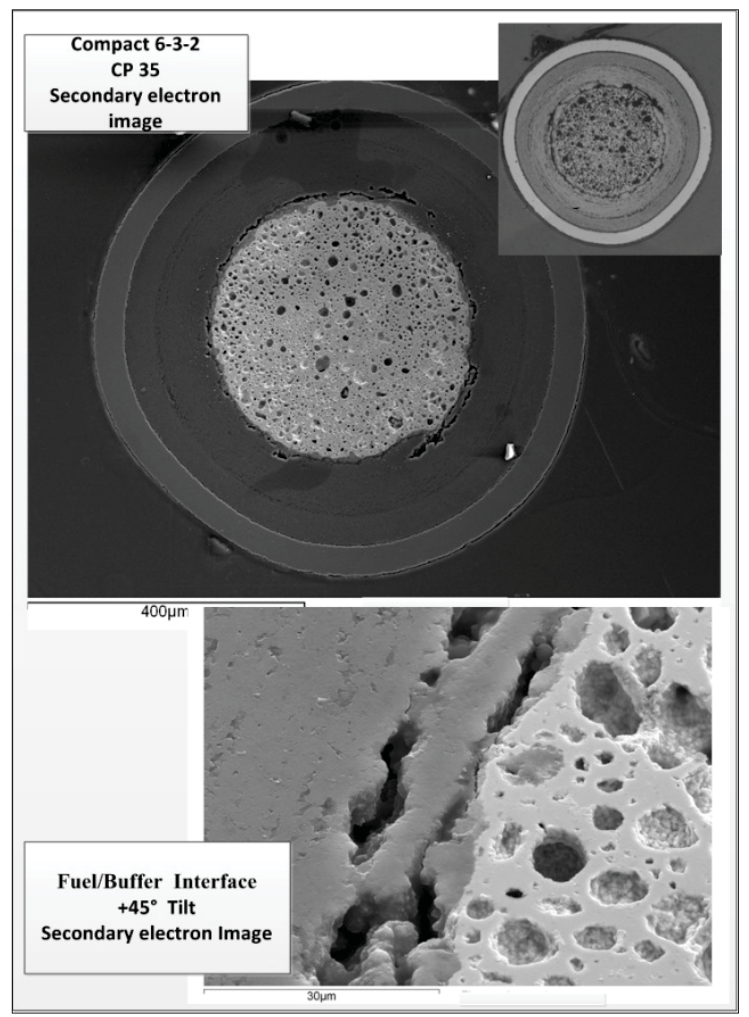

Figure 38. Secondary electron images of CP 35 from Compact 6-3-2.

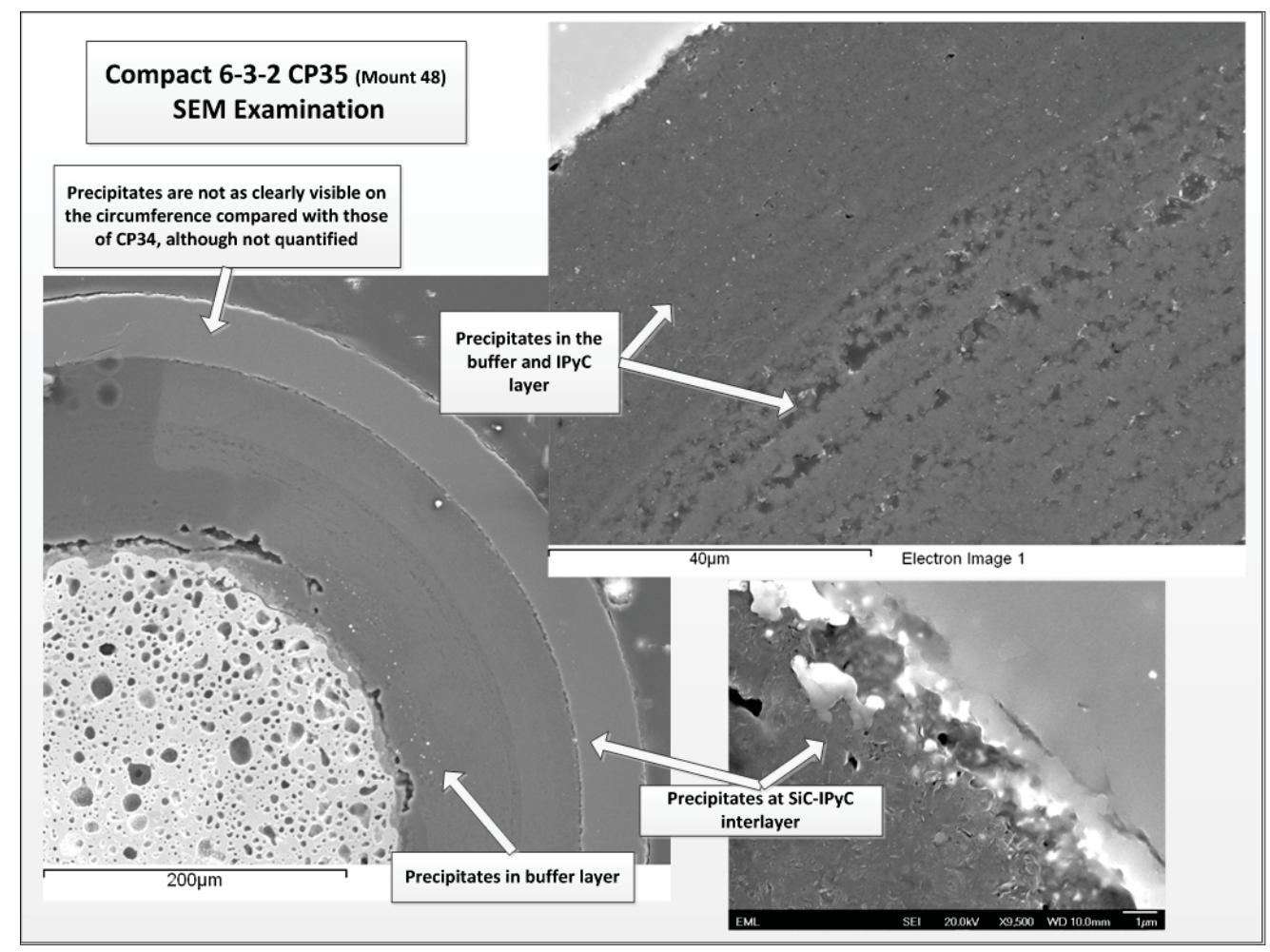

Figure 39. SEM micrographs of CP35 of Compact 6-3-2 showing precipitates (Pd-rich) in the buffer, IPyC layer and at the SiC-IPyC interlayer. 


\subsubsection{FIB sample preparation}

FIB specimens were prepared from CP35 and identified by the numbers shown in Figure 40. Only the specimen from Position 6 will be discussed in this report (specimens from Position 7 will be discussed in a separate fuel kernel report). As shown in Figure 40, the specimen is a thin planar monolith cut perpendicular to the polished particle surface and was oriented tangent to the SiC-IPyC interface in a region where significant precipitates were found during the SEM analysis. Only one TEM sample was prepared from CP35 because a significant number of TEM samples were prepared from CP34, which also had a higher than average Ag- $110 \mathrm{~m} / \mathrm{Cs}-137$ activity ratio, and therefore is presumed to have experienced a similar level of Ag release as described in Section 3.1.

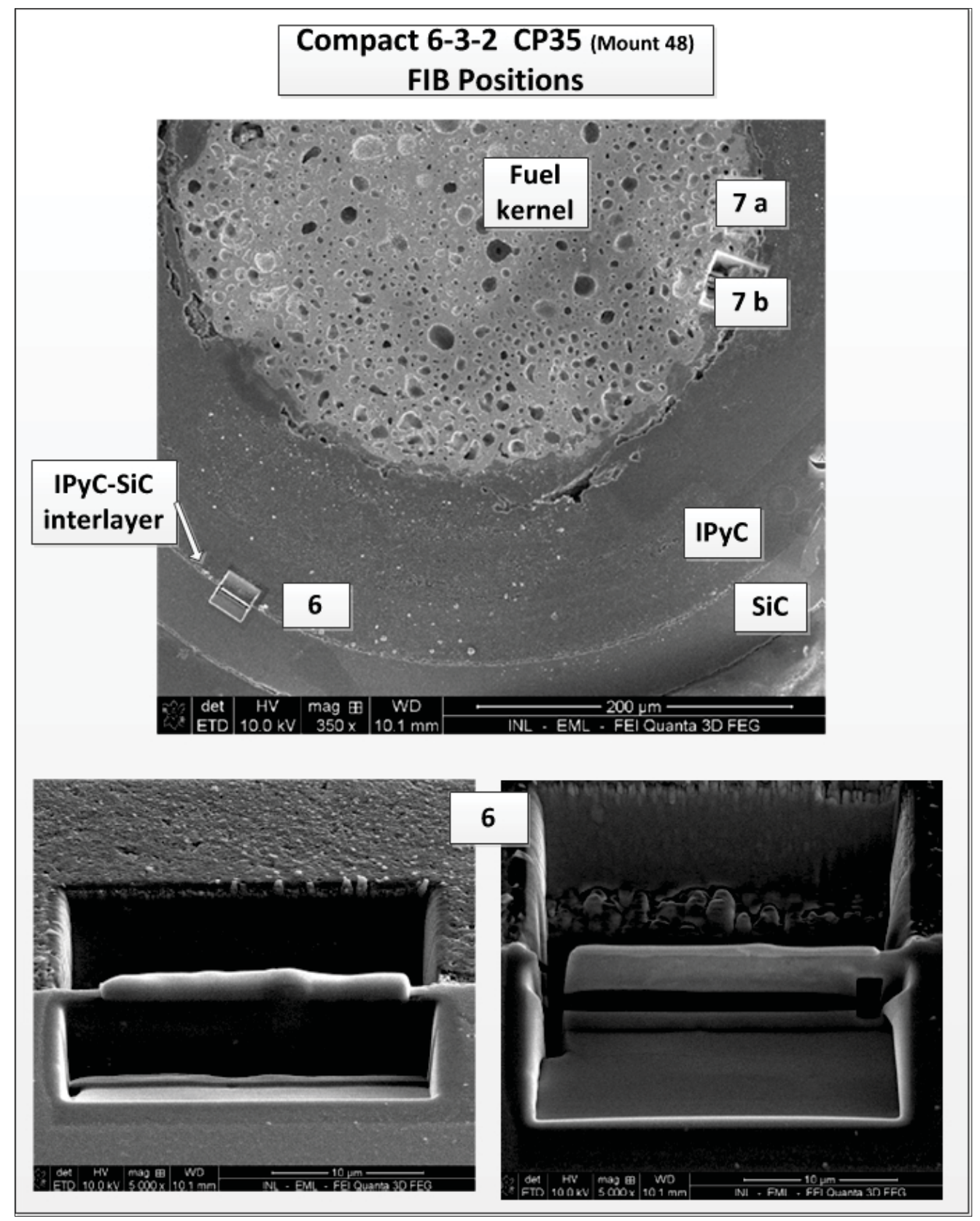

Figure 40. FIB sample preparation detail of CP35 from Compact 6-3-2. 


\subsubsection{TEM Examination}

\subsubsection{Initial TEM Analysis}

Figure 41 shows BF TEM micrographs with corresponding EDS spectra, indicating Si-Pd-U precipitates in the SiC layer in TEM Position 6. The precipitates found in CP35 are similar to those discussed in Section 4.1.3.2, and zone-axis diffraction patterns from precipitates in CP35 are indexed as $\mathrm{UPd}_{2} \mathrm{Si}_{2}$. In contrast to the areas evaluated for CP34, cesium was also identified along with Pd in some areas, as shown in Figure 42. It appears that the Cs-containing precipitates are not located on the grain boundaries only, but within individual grains. Based on the BF TEM micrograph with EDS spectrum, Figure 43 shows that the Cs-containing precipitates are not located on the grain boundaries only, but within individual $\mathrm{SiC}$ grains of CP35 at Position 6 . However this needs to be confirmed with further data analysis. Further detailed TEM examination on additional samples will also provide more information on this "wormlike" feature, and if it may actually be a crack. It is further noted that indications of $\mathrm{Zr}$ have also been found in these Cs-containing precipitates.

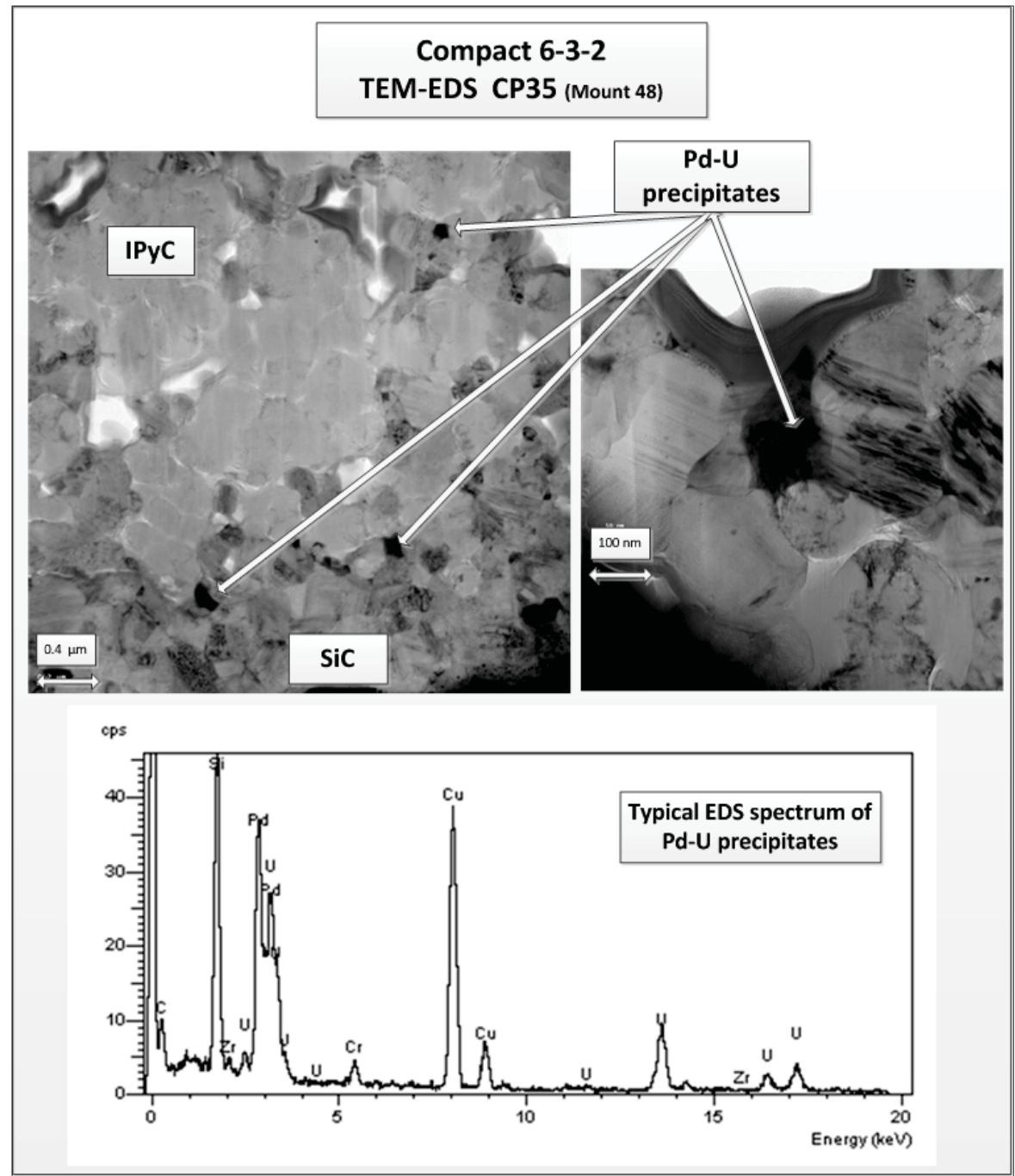

Figure 41. TEM micrograph showing Pd- U precipitates in the SiC layer in FIB Position 6 for CP35 from Compact 6-3-2. 


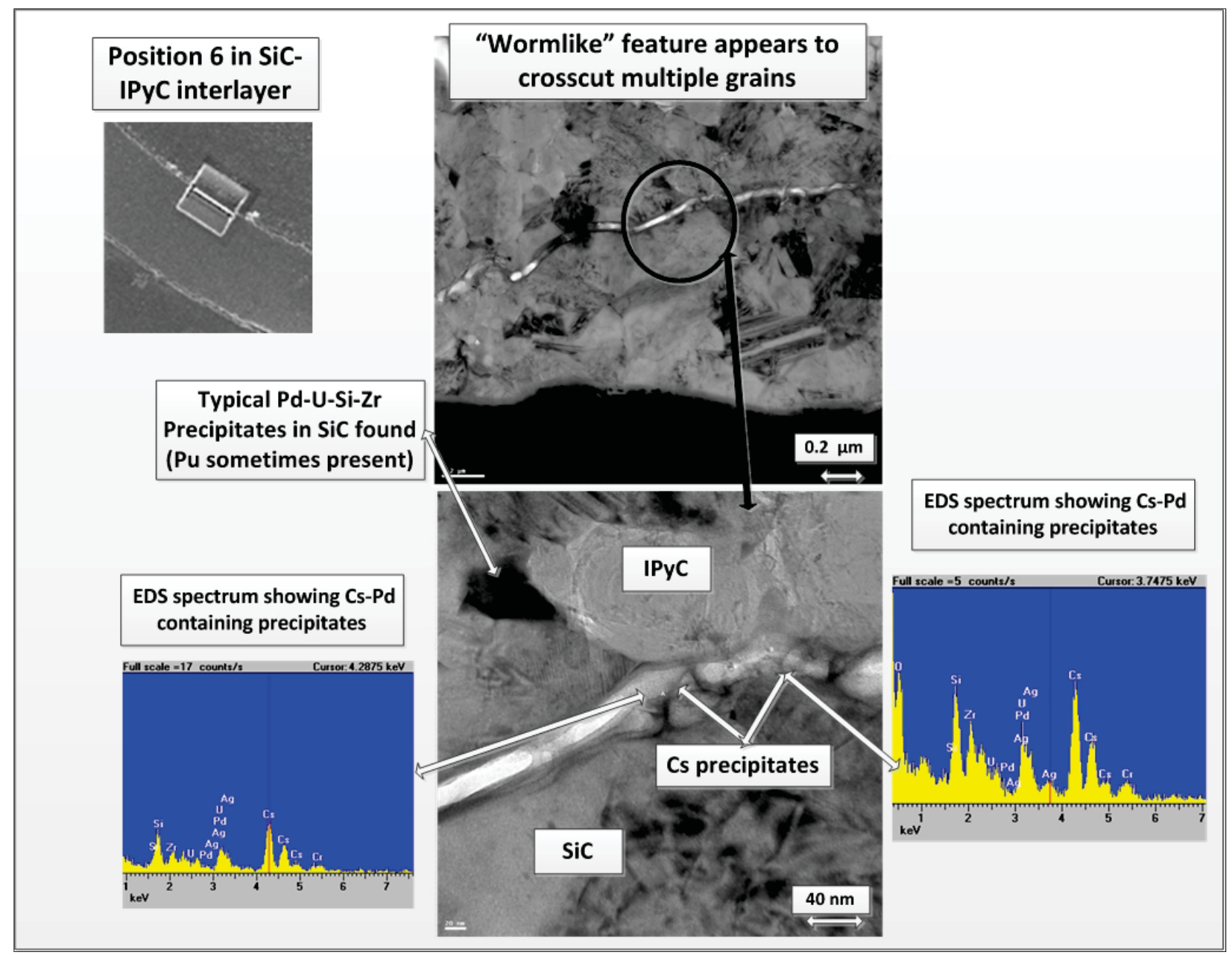

Figure 42. BF TEM micrograph showing Cs precipitates in the wormlike structure and SiC grain (Note: Ag is not confirmed due to peak overlap). 


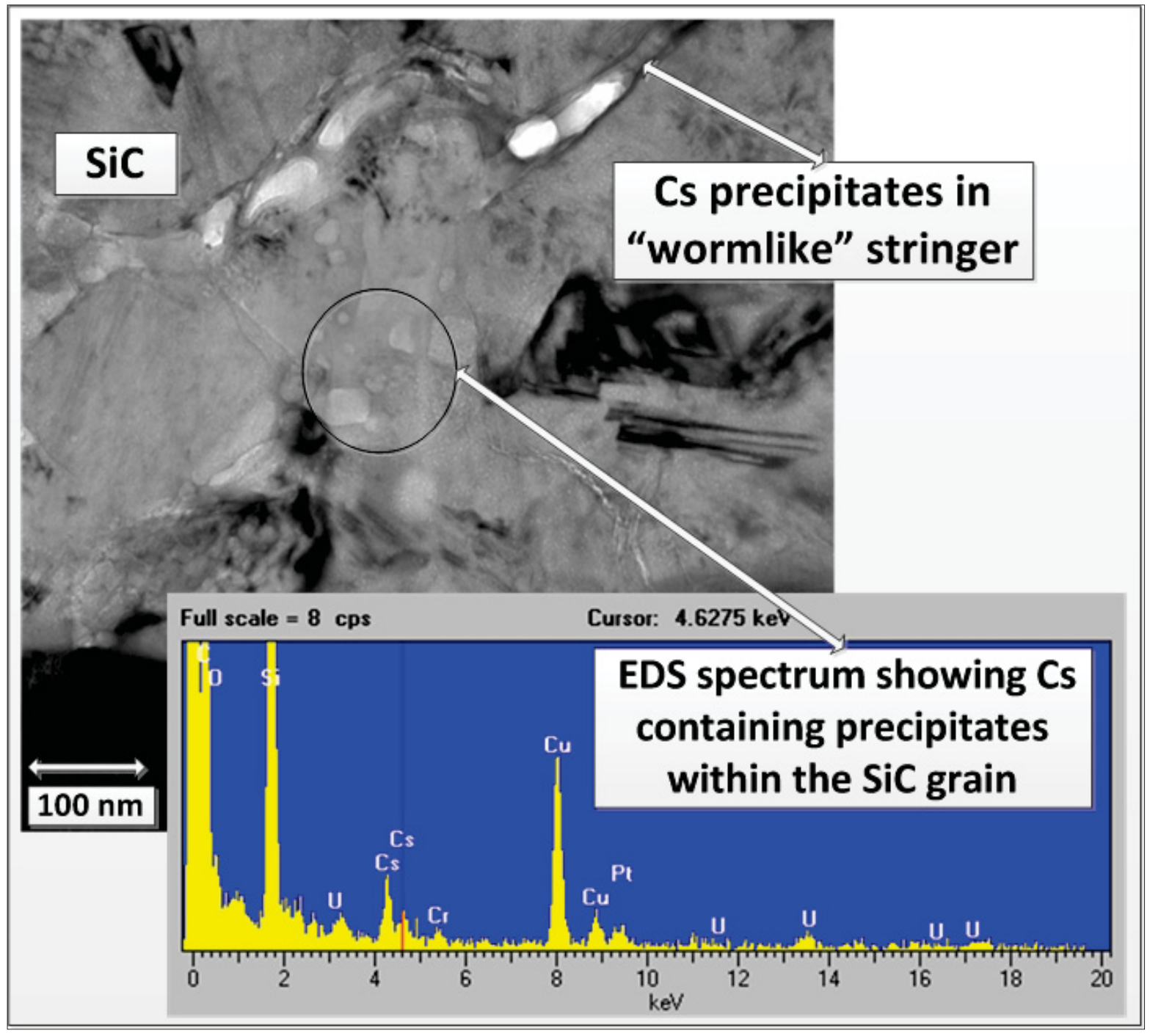

Figure 43. BF TEM micrograph with EDS spectra showing apparent Cs-containing precipitates in individual SiC grains of CP35 at Position 6.

\subsubsection{Irradiation Effects on SiC and PyC Microstructures}

\subsubsection{SiC Layer}

No evidence was found of any phase transformation from the cubic to the hexagonal phase because of the irradiation cycle as determined by SAD patterns obtained on the different TEM samples prepared from CP35. Examples of representative SAD patterns are presented in Figure 44. 


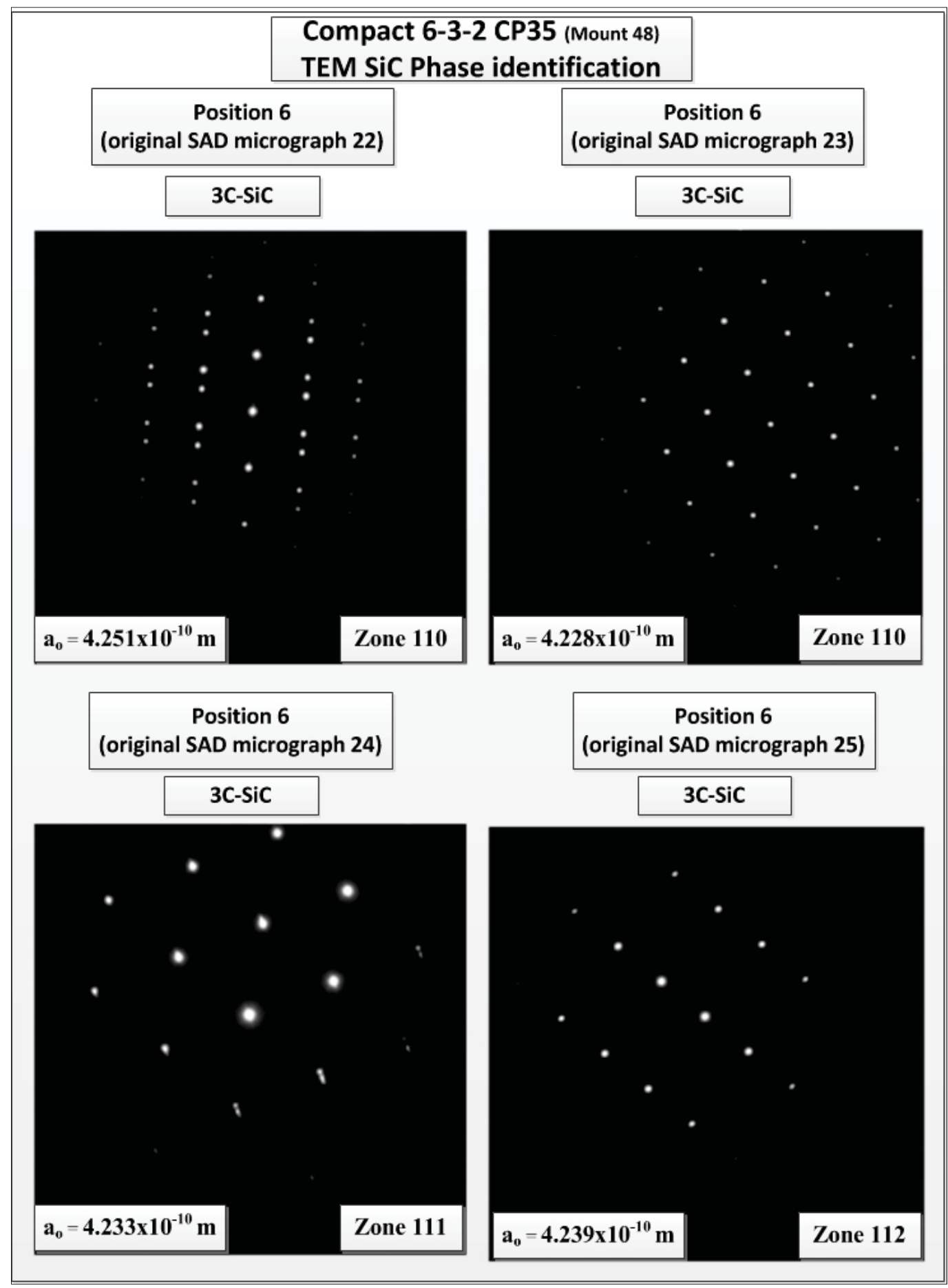

Figure 44. Summary of representative selected area diffraction patterns of the SiC layer of CP35 determined at different positions.

Figure 45 shows that the cavities are of angular shape, which contrasts with the cavities found in CP34. Kondo et al. ${ }^{12}$ also found that the shape of cavities changes from spherical to facetted as a function of irradiation temperature. If compared with the spherical cavities found in CP34 (Figure 33d), these results also agree with the findings of the IPyC selected area diffraction patterns, which also show that CP35 may have been exposed to higher irradiation temperatures compared to CP34. 


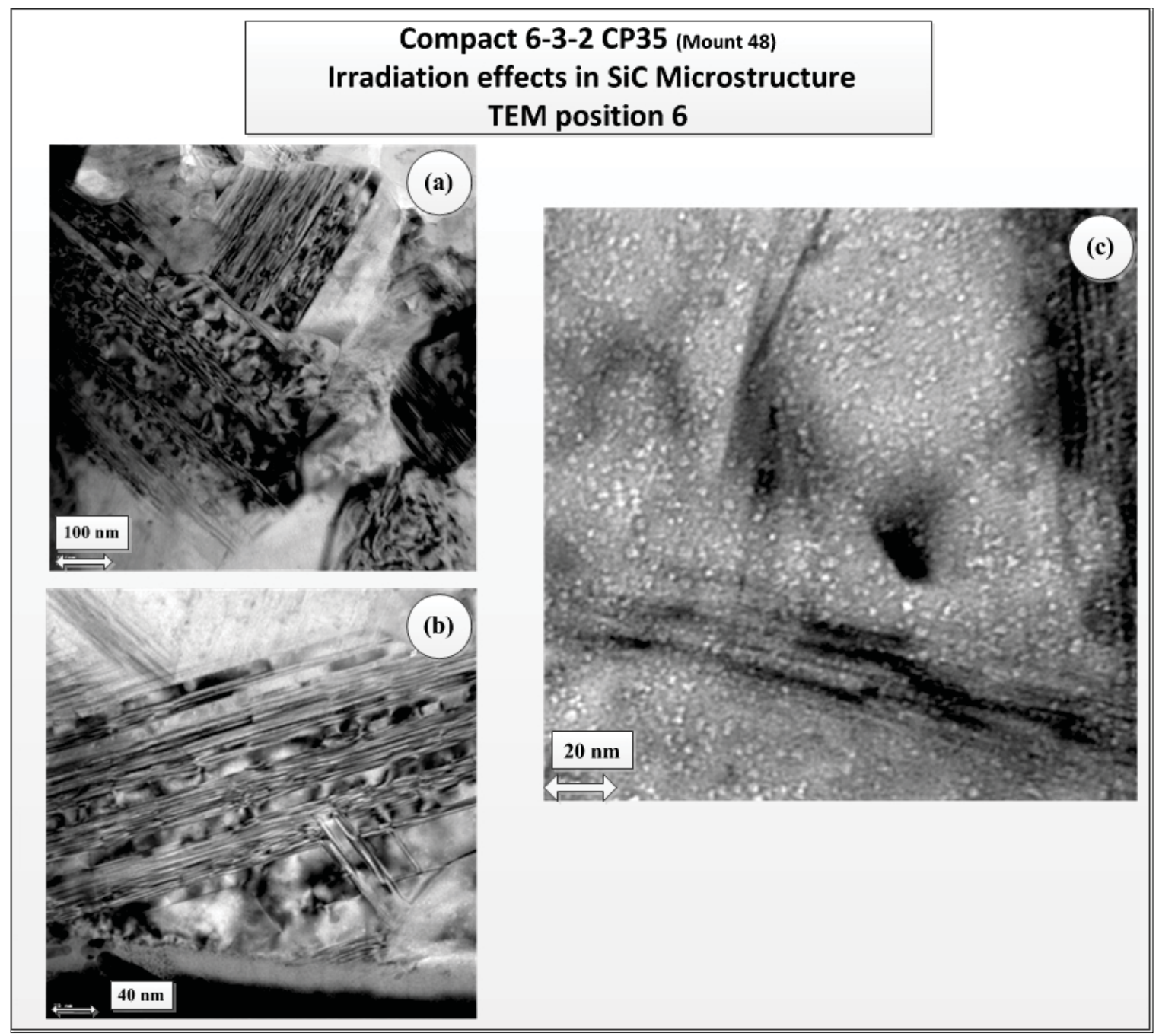

Figure 45. Bright field TEM images of the irradiated SiC microstructure of CP35 showing (a) typical loops because of irradiation, (b) higher magnification shows the collection of loops at the stacking faults and (c) showing that the cavities are angular shaped.

\subsubsection{IPyC layer}

Only one selected area diffraction pattern was measured for the IPyC of CP35 and is shown in Figure 46. The intensity variations in the diffraction ring pattern for CP35 are more prominent if compared with those of the irradiated IPyC from CP34 (Figure 36) and the unirradiated IPyC from AGR-1 experiment (Figure 34), however this needs to be verified as the specific aperture sizes were not recorded during the time of analysis and may not be the same for all of the diffraction patterns. In the case of comparative aperture sizes, the intensity variations observed in Figure 46 may suggest that CP35 has been exposed to higher temperatures than CP34 based on the previous work by Van Rooyen et al. ${ }^{13}$ As in the discussion on CP34, it can't be concluded whether temperature or neutron fluence is the predominant reason for the observed changes as more comparative studies need to be completed with other irradiated compacts from the AGR-1 experiment and as well as data published in the literature. Future studies will include comparative Bacon Anisotropy Factor values and diffraction pattern ring measurements to enable quantification of anisotropy. 


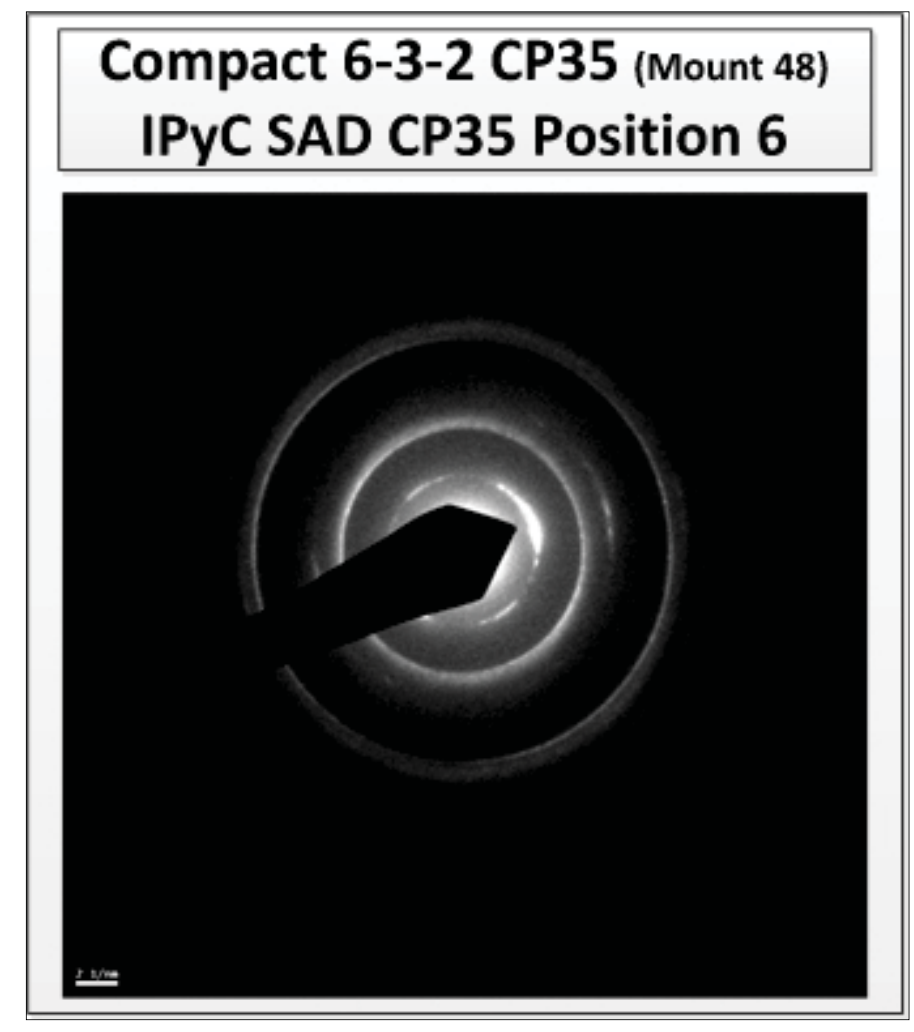

Figure 46. Selected area diffraction pattern as preliminary data on IPyC structure of CP34 Position 6. (aperture size was not recorded during time of analysis and may not be the same for all the diffraction patterns).

\subsubsection{IPyC-SiC Interface Integrity}

No evidence was observed of IPyC-SiC interface debonding due to irradiation in CP35 in the TEM sample (Position 6) investigated as shown in Figure 47. 


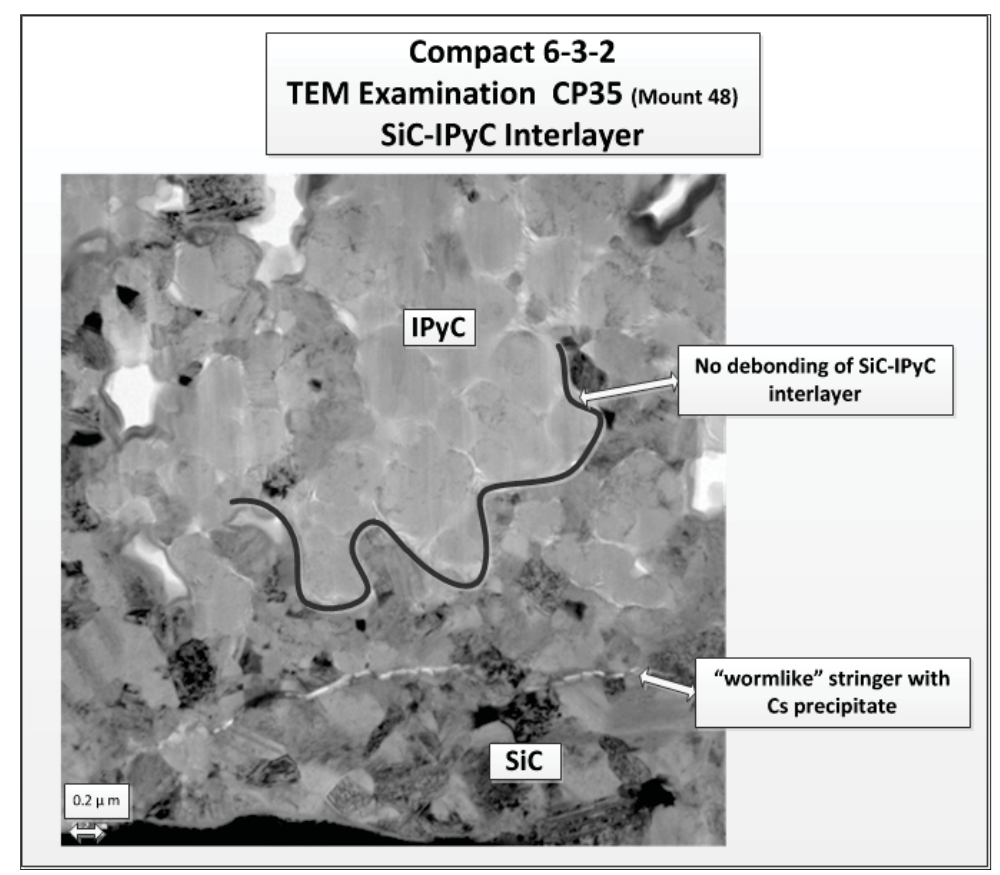

Figure 47. BF TEM image of the SiC-IPyC of CP35 shows no evidence of debonding.

\section{$4.3 \quad$ CP39}

\subsubsection{SEM examination}

The electron microscopic examination of CP39 was concluded after a cursory SEM examination because of the surface condition of the mounted sample. Figure 48 shows that the surface is contaminated with loose debris, which most probably occurred during the decontamination process in EML. At this stage, no further work is planned, as both CP34 and CP35 provided uncontaminated mounts for further evaluation. It is however important to determine the cause of this contamination, to prevent reoccurrences during future decontamination activities. 


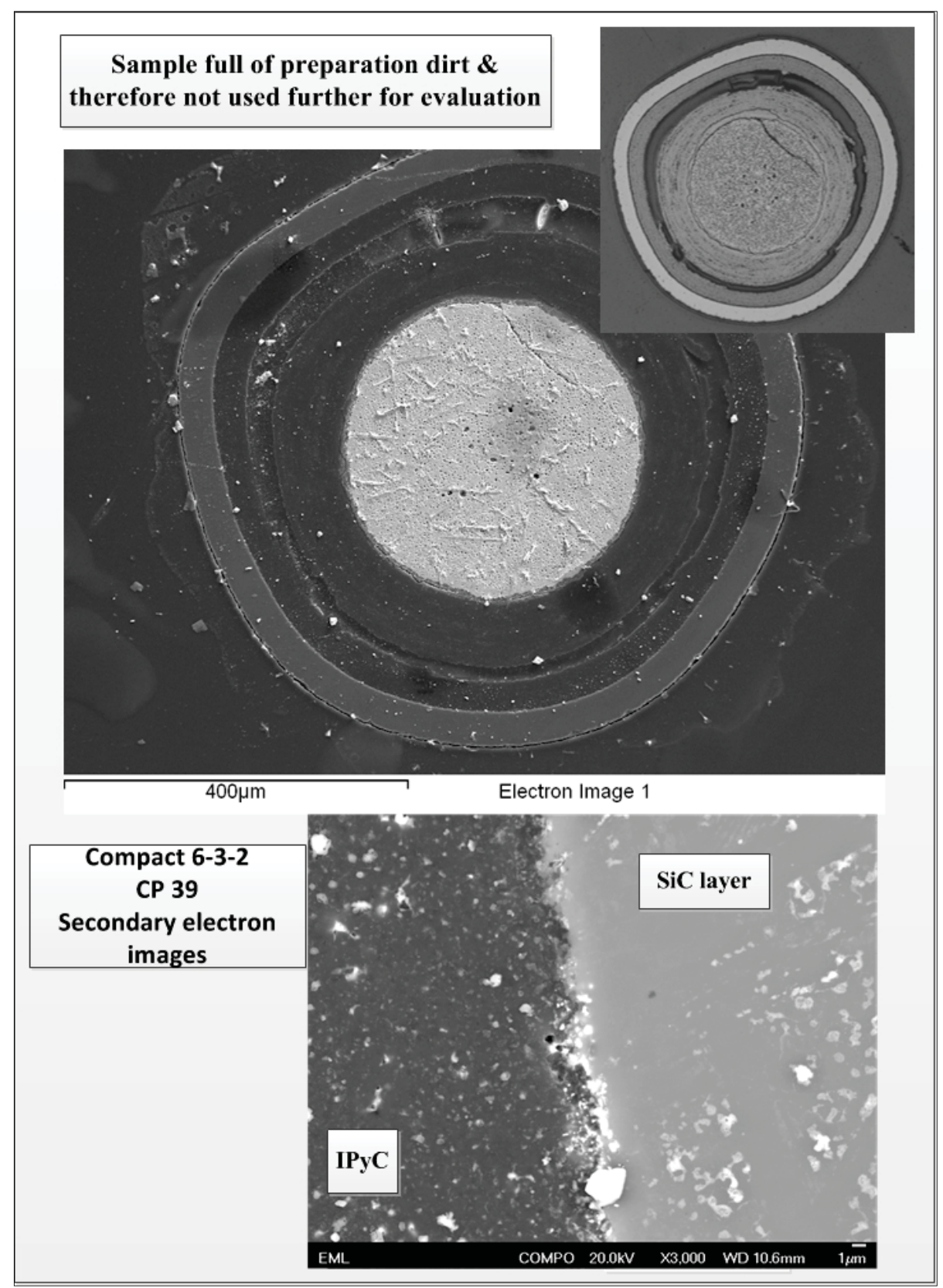

Figure 48. Back scattered electron images of CP 39 of Compact 6-3-2, showing surface contamination. Inset at upper right is an optical micrograph.

\subsection{CP30}

\subsubsection{SEM Examination}

A single particle from Compact 6-3-2 with an Ag-110m inventory at the low end of the distribution (indicative of significant in-pile Ag release) was mounted and polished for microanalysis. The objective was to determine if there are observable and quantifiable differences in the nature of the coating layers or the distribution of fission products in the coating layers that correlate with the level of $\mathrm{Ag}$ retention in the particle. Unfortunately, complications were encountered during grinding and polishing of this particle, 
resulting in damage to the particle cross-section as discussed in Section 3.3.1. Figure 49 shows the SEM image of the only piece of sample left from CP30 for examination and no visible fission product precipitates could be observed. However, it was decided to proceed with FIB/TEM analysis of the mount because it was the only sample available from Compact 6-3-2 with a measured low Ag-110m inventory (low $\mathrm{Ag}-110 \mathrm{~m} / \mathrm{Cs}-137 \mathrm{ratio}$ ) and, since the sample preparation exposed the $\mathrm{SiC}$ layer very near to the IPyC interface, it was still expected to yield information about fission product precipitates and $\mathrm{SiC}$ microstructure that would be of value as a comparison to CP34 and CP35.

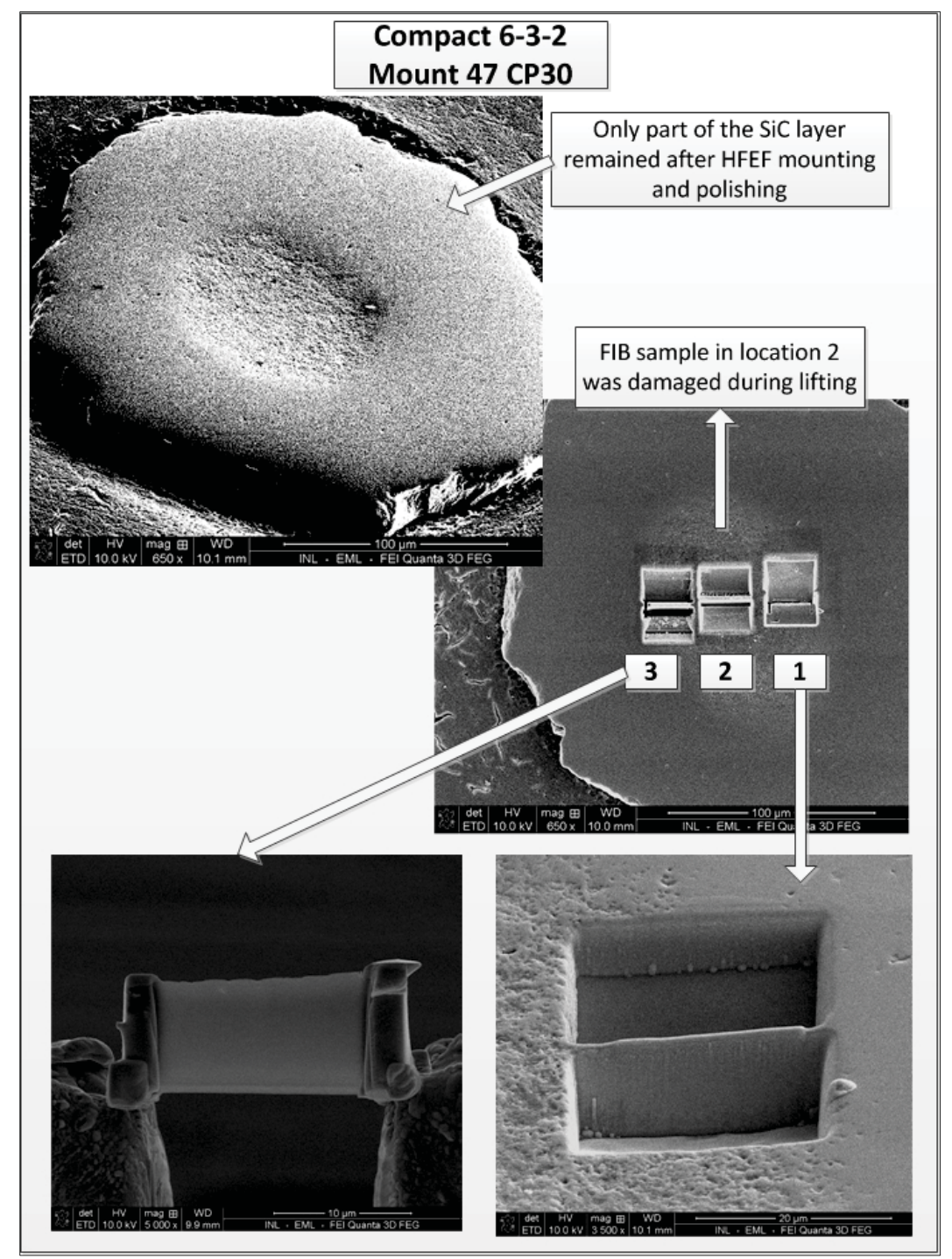

Figure 49. FIB sample preparation position for CP30 from Compact 6-3-2.

\subsubsection{FIB sample preparation}

TEM analysis was completed on FIB samples from Positions 1 and 3 as shown in Figure 49. The TEM sample in Position 2 was damaged during lifting and therefore not included in the evaluation. 


\subsubsection{TEM Examination}

\subsubsection{Initial TEM Analysis}

The TEM analysis shown in Figure 50 revealed typical irradiated $\mathrm{SiC}$ microstructure with Pd-rich nano precipitates. Columnar grains were also detected at Position 1 in contrast with previous TEM examinations on CP34 and CP35 as shown in Figure 50. This may be because of the different orientation of this FIB lift-out relative to the others (ion milling for this FIB lift-out was performed in a direction roughly parallel to the particle radius). Only nano-scale precipitates were found in these two samples as shown in Figure 50.

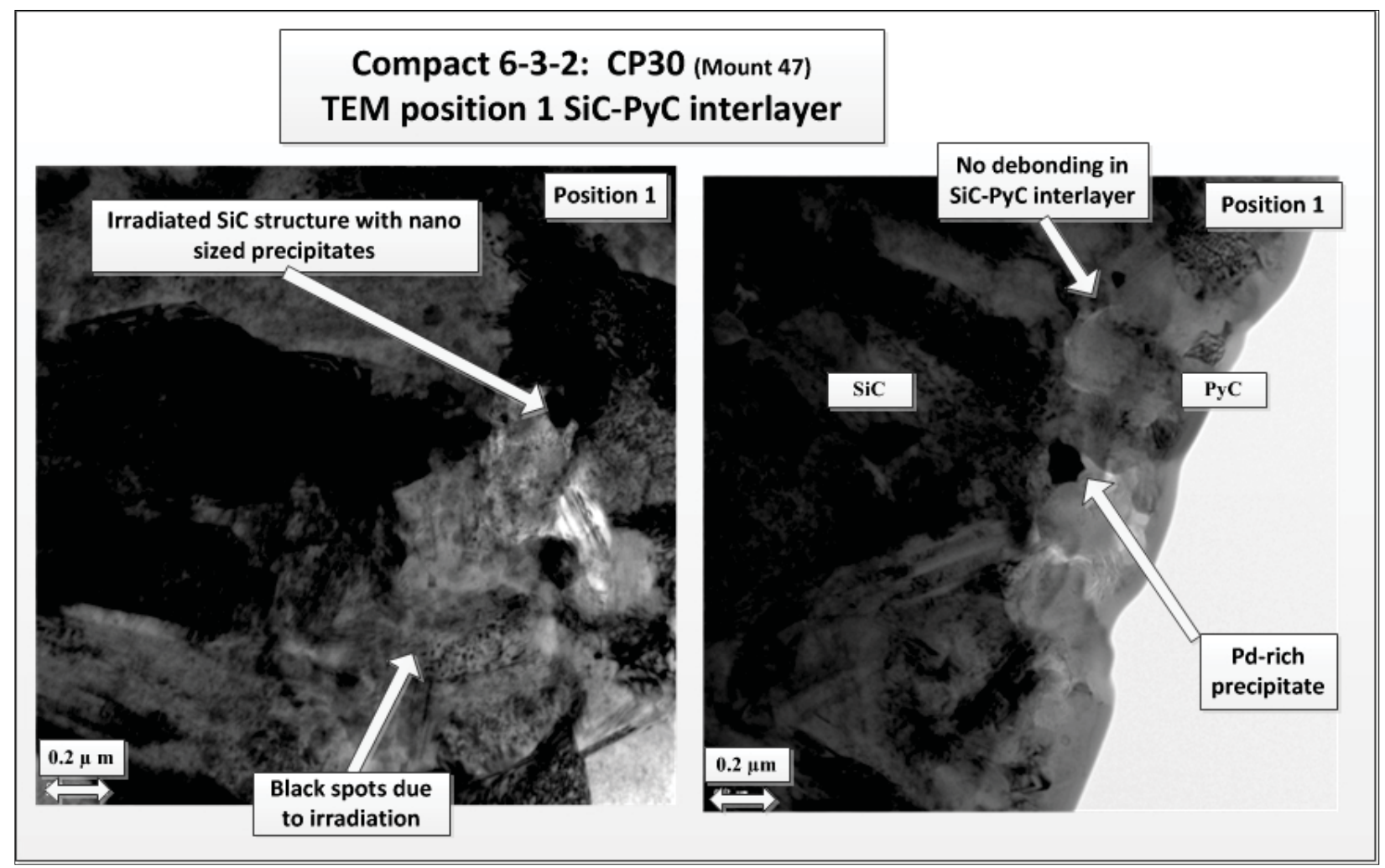

Figure 50. TEM micrograph showing representative images of precipitates in the SiC layer of particle 30 from Compact 6-3-2, TEM position 1. Bright field TEM images of the irradiated SiC microstructure of CP30 showing (left) typical black spots (representative of dislocation loops) because of irradiation and (right) no debonding of the SiC-PyC interlayer.

\subsubsection{Precipitate Identification}

All of the TEM data on high Pd-precipitates in CP30 are from a single precipitate whose diffraction pattern, shown in Figure 51, can be indexed as a slightly off-axis pattern from the [010] zone of $\mathrm{UPd}_{2} \mathrm{Si}_{2}$ (PDF4+ card number 00-047-1029). Faint reflections indicated by arrows in Figure 51 may correspond to those observed in [110] diffraction patterns from precipitates in other samples (e.g., Figure 25). The EDS spectrum from this precipitate was not quantified. Qualitative comparisons suggest that proportions of U, $\mathrm{Pd}$, and $\mathrm{Si}$ are comparable to those from precipitates in $\mathrm{CP} 34$ and $\mathrm{CP} 35$. However, this spectrum is unique in that it does contain very small concentrations of $\mathrm{Pu}$ in some precipitates ( 0 to $1.35 \mathrm{wt} \%)$. 


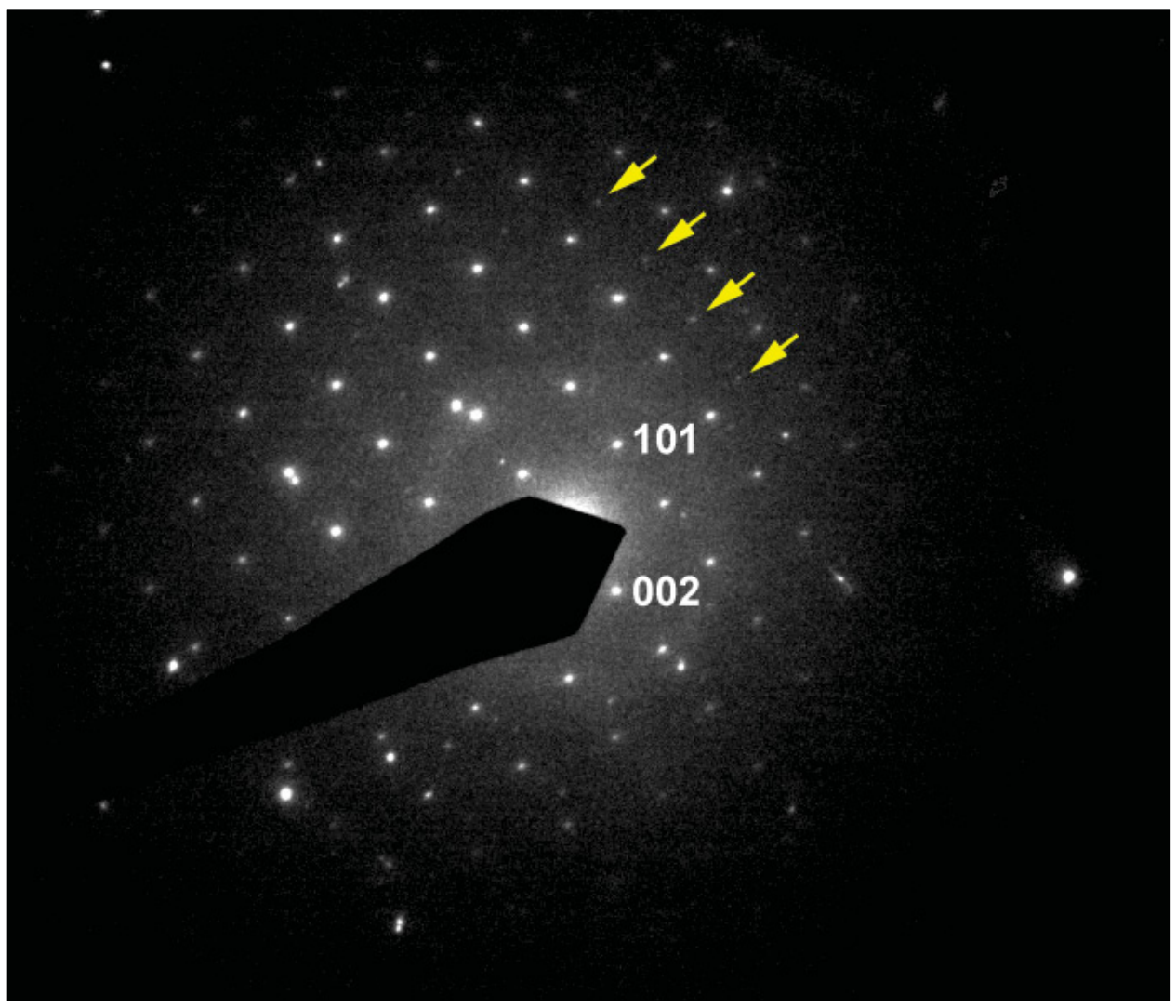

Figure 51. Diffraction pattern indexed as the [010] zone axis of $\mathrm{UPd}_{2} \mathrm{Si}_{2}$.

\subsubsection{Irradiation Effects on SiC and PyC Microstructures}

\subsubsection{SiC Layer}

Three SAD patterns of the SiC from different areas on TEM sample Position 1 were analyzed and results are presented in Figure 52. Two of these SAD patterns represent the cubic SiC phase while the other pattern is most probably from a hexagonal phase, but this needs to be confirmed. At this stage it is not possible to state that the hexagonal phase identified is a result of neutron irradiation, as it is known from previous studies by Van Rooyen et al. ${ }^{13}$ that small amounts of hexagonal SiC may exist because of the manufacturing conditions. It is thus recommended that conclusions on this finding be reserved until more statistical data is available. It is also recommended that unirradiated AGR-1 specimens be examined with the same methods in order to determine if any hexagonal $\mathrm{SiC}$ is present. It is expected that the EBSD analysis of these layers will provide more statistical data to determine if hexagonal phases form during neutron irradiation.

Figure 50 shows the BF field TEM images of the irradiated $\mathrm{SiC}$ microstructure of CP30 showing (a) typical black spot loops because of irradiation and (b) no debonding of the SiC-PyC interlayer.

Unfortunately, no higher magnification images were taken, so more detail about the densities of the loops cannot be provided. However, no cavities were observed on the areas examined. These two observations may suggest that CP30 was exposed to lower temperatures then CP34 and CP35. However, additional TEM micrographs need to be examined for validation purposes. 


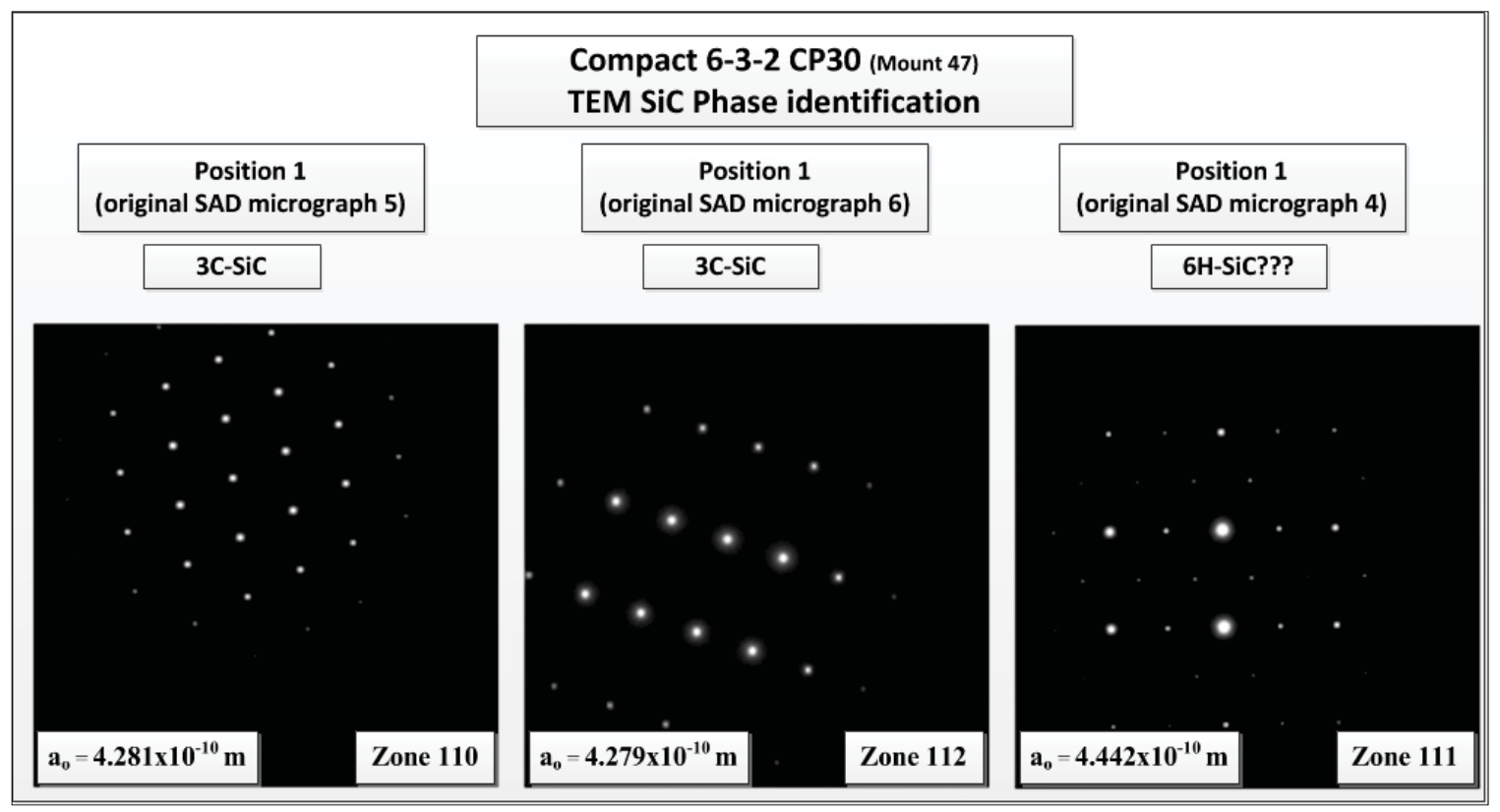

Figure 52. Summary of representative selected area diffraction patterns of the SiC layer of CP30 determined at different positions.

\subsubsection{IPyC Layer}

Because of the sample preparation complications discussed previously, a detailed examination of the IPyC layer was not completed.

\subsubsection{IPyC-SiC Interface Integrity}

Because of the sample preparation complications discussed previously, only a very small section of the IPyC-SiC interlayer was available for evaluation and no debonding was observed in that region. 


\section{QUALITY}

\subsection{Quality Requirements}

The work reported in this report was performed under the governance of VHTR TDO Quality Assurance program. ${ }^{14}$

\subsection{Storage of Samples and Digital Data}

The FIB prepared CPs for TEM investigations were kept in numbered plastic storage/transport racks in the TEM room. Samples are identified using the grid position number and was recorded in the laboratory note book. These samples are secured in the TEM laboratory in the Electron microscopy laboratory which is access controlled. A laboratory notebook (Note book \# E-102) with specific CP numbers and FIB location numbers was kept so that any specific particle from any batch can easily be located, should further investigations be required. Additionally, the specific microscopist kept a laboratory notebook for recording purposes.

Electron microscopy digital data, as well as interpreted data used in the electron microscopy technical reports will be stored on the INL Electronic Document Management System at the time of their completion. Until the reports are completed, the original data will be stored on the specific microscope's hard drive as well as on a dedicated VHTR/AGR drive. Additionally, these raw data will also be stored on the $\mathrm{X}$ drive of the Electron Microscopy lead principal Investigator). The two latter drives are back-up periodically as part of INL back-up policy.

\subsection{Skill of Performers (Electron Microscopists) and Principal Investigator}

All electron microscopic work was performed by skilled operators after assessment was performed in accordance with the internal Materials and Fuels Complex EML training records. ${ }^{15,16,17}$

All electronic microscopic interpretation was performed by the electron microscopist and/or the electron microscopy principal investigator and peer reviewed by relevant technical experts in this field.

\subsection{Equipment and Software}

Data about individual precipitates were collected using the JEOL 2010 TEM in the EML at INL, operating at a nominal voltage of $200 \mathrm{kV}$. Images and diffraction patterns were collected digitally with a Gatan Ultrascan camera and Gatan Digital Micrograph software, v. 1.85.1535, license ID 300436478 with a Dell Optiplex GX620 Pentium 4 CPU, 2.8 GHz, running under Windows XP Professional, version 2002, Service Pack 3

EDS spectra were collected up to the end of 2011, using an Oxford Link Petafet EDX detector with a SiLi crystal, nominal $20 \mathrm{eV}$ channel width, nominal energy range from 0 to $20 \mathrm{keV}$, and nominal $136 \mathrm{eV}$ resolution. The spectra were collected and qualitatively analyzed using Link ISIS software, ISIS Suite Revision 3.2 with a HP Pentium I processor computer using Windows 95 operating system.

EDS spectra were collected from January 2012 using a Bruker $133 \mathrm{eV}$ silicon drift detector and Quantax 200 Esprit 1.9 software, v. 1.9.3.3047, 9/13/11, product code 200 system 2752 with a Dell Optiplex 780, Core 2 duo CPU, E8400@3.00 GHz 3.00GHZ using Windows 7 operating system.

Phase identifications in TEM data were done by comparing experimental measurements of compositions, d-spacings, and (if appropriate) interplanar angles to the current version of the International 
Centre for Diffraction Data's PDF4+ database, supplemented by literature research on specific phases if needed.

The EDS and WDS work on the JEOL 7000F SEM was collected using the Oxford Instruments INCA suite, version 4.09. Detector calibration was performed in November 2011 by the Oxford Instruments service engineers during the preventative maintenance visit. All records of calibration are held in the EML as part of the maintenance records. 


\section{CONCLUSIONS}

\subsection{Precipitate Identification}

A newly developed quantification approach on the SEM precipitate location yielded the following results for $\mathrm{CP} 34$ :

- Clusters of Pd-rich precipitates were identified around the full circumference in and in close proximity to the SiC-IPyC interlayer up to an average $\mathrm{SiC}$ and IPyC depth of $6.74 \mu \mathrm{m}$ and $2.88 \mu \mathrm{m}$ respectively. It was found that the maximum depth of $\mathrm{Pd}$-rich precipitate penetration in the $\mathrm{SiC}$ was $15.25 \mu \mathrm{m}$. TEM examination of the SiC layer indicated an approximate depth of penetration that agrees with this value.

- The SiC-IPyC interlayer had an average thickness of $1.7 \mu \mathrm{m}$.

This investigation found Pd-precipitates in the IPyC layer as well as in the $\mathrm{SiC}$ up to a maximum depth of $15.25 \mu \mathrm{m}$. These findings differ from those reported to date on the HFR-EU1bis experiments $(10.2 \%$ FIMA, $1523 \mathrm{~K})$, which found Pd present in the IPyC layer at the inside of the SiC layer, but not in the $\mathrm{SiC}$ layer itself.

All diffraction patterns from precipitates in the $\mathrm{SiC}$ layer in CP30, CP34, and CP35 could be produced by single crystals of $\mathrm{UPd}_{2} \mathrm{Si}_{2}$. Qualitative analyses of the EDS spectra showed significant concentrations of $\mathrm{U}, \mathrm{Pd}$, and $\mathrm{Si}$, with some spectra also having significant concentrations of $\mathrm{C}$ and $\mathrm{Zr}$. Semiquantitative analyses of normalized atomic percentages of $\mathrm{Si}, \mathrm{Pd}$, and $\mathrm{U}$ showed similar compositions, with 40 at $\% \mathrm{Si}, 45$ at $\% \mathrm{Pd}$, and 10 to $15 \mathrm{at} \% \mathrm{U}$. This similarity in proportions of $\mathrm{U}, \mathrm{Pd}$, and Si suggests that these elements occurred in the same phase. Relative intensities of some reflections differed between diffraction patterns from different precipitates in the present data, as well as between different cards for $\mathrm{UPd}_{2} \mathrm{Si}_{2}$ in the PDF4+ database. These differences are not considered significant for phase identification.

Many images of precipitates show dark spots within the precipitates, which may indicate variations in internal structure. Diffraction patterns commonly show extra reflections, which may be from double diffraction. Further detailed analysis is required to understand both the internal structure of the precipitates and the origin of the extra reflections.

Further investigation is also required to identify the phase containing $\mathrm{Zr}$, which is present in significant concentrations in some precipitates but is absent in spectra from other precipitates. This variation in the concentration of $\mathrm{Zr}$ relative to those of $\mathrm{U}, \mathrm{Pd}$, and $\mathrm{Si}$ suggests that the $\mathrm{Zr}$ is in a different phase, and that EDS spectra with $\mathrm{Zr}$ represent mixtures of $\mathrm{UPd}_{2} \mathrm{Si}_{2}$ with a $\mathrm{Zr}$-bearing phase. Even though $\mathrm{Zr}$ is a $4 \mathrm{~d}$ transition metal, its atomic radius is significantly different from that of $\mathrm{Pd}$, and an extensive $\mathrm{U}(\mathrm{Zr}, \mathrm{Pd})_{2} \mathrm{Si}_{2}$ solid solution seems unlikely. $\mathrm{U}$ and $\mathrm{Pu}$ often substitute for one another in metals and intermetallics, and it seems reasonable to speculate that low concentrations of $\mathrm{Pu}$ could substitute for $\mathrm{U}$ in $\mathrm{UPd}_{2} \mathrm{Si}_{2}$. Zr can also be an artifact in EDS spectra collected with the objective aperture inserted, and therefore recent analysis is recorded as such.

It is not possible to determine from the current data obtained from the L $\alpha$ x-rays whether low concentrations of Ag are present in the precipitates. Since Ag and Pd have identical atomic radii (both $0.144 \mathrm{~nm}$ for the pure material), it therefore seems reasonable to speculate that any Ag atoms that might be present would substitute for $\mathrm{Pd}$ atoms in a $\mathrm{U}(\mathrm{Ag}, \mathrm{Pd})_{2} \mathrm{Si}_{2}$ solid solution rather than forming a separate phase.

Cesium was also identified along with Pd in CP35 in contrast to the areas evaluated for CP34. It appears that the Cs-containing precipitates are not only located on the grain boundaries, but also within 
individual grains; however, this needs to be confirmed with further analyses of the data. It is further noted that indications of $\mathrm{Zr}$ have also been found in areas containing Cs.

\subsection{Irradiation Effects on Microstructure}

No evidence was found of any phase transformation of $\mathrm{SiC}$ from the cubic to the hexagonal phase because of irradiation as determined by SAD patterns measured on the different TEM samples prepared from CP34 and CP35. Although possibly one hexagonal structure for CP30 was observed, it is not possible to state that this hexagonal phase formed as a result of irradiation, as it is known from previous studies that small amounts of hexagonal $\mathrm{SiC}$ may exist in cubic $\mathrm{SiC}$ because of manufacturing conditions. It is thus recommended that conclusions based on this finding be reserved until more statistical data are available, and it is expected that the EBSD analysis of these layers will provide more statistical data to determine if hexagonal phases formed during irradiation.

Intensity variations in the IPyC diffraction ring patterns after irradiation are observed for CP34 and $\mathrm{CP} 35$ and are an indication that the $\mathrm{PyC}$ is becoming anisotropic. Intensity variations within CP34 are not significant, although a difference is observed between those for CP34 and CP35 as well as between these two irradiated specimens and those of unirradiated material. However, as the SAD apertures were not recorded, this needs to be verified. At this point, it cannot be concluded whether temperature or the neutron fluence is the predominant reason for the observed changes. However, previous research implies that it may be predominantly because of the temperature effect.

Although a full investigation was not completed on the loop and dislocation densities of this material, no significant difference between the inner and outer $\mathrm{SiC}$ portions of the CP34 was observed in a specific position, although differences were observed between those at different locations on the circumference of the $\mathrm{SiC}$ layer. No denuded zone for dislocation loops along grain boundaries were observed during this study, but previous studies have typically reported denuded zones at grain boundaries in some areas for irradiated $\mathrm{SiC}$ at $1130^{\circ} \mathrm{C}$. This may be important while studying the fission product transport mechanisms and therefore will be investigated specifically in the areas where fission product precipitates were found. At this point no conclusion is reached from this observation and will need to be quantified during further analysis. The cavities were predominantly found to be spherical in shape for CP34 in contrast with angular shaped cavities found in CP35. Other researchers typically find that the shape of cavities change from spherical to facetted as a function of irradiation temperature.

Both the findings on the IPyC diffraction patterns and the cavity shape differences suggest that CP35 may have been exposed to higher temperatures than that of CP34.

No debonding or macroscopic cracks were observed in the SiC-IPyC interlayer in all the samples of CP34, CP35 and CP30 examined in this study.

\subsection{Summary of Results}

Table 5 summarizes the results of this study. 
Table 5. Study results.

\begin{tabular}{|c|c|c|c|c|}
\hline $\mathrm{SiC}$ & $\mathrm{CP} 34$ & $\mathrm{CP} 35$ & CP39 & $\mathrm{CP} 30$ \\
\hline Phases of $\mathrm{SiC}$ & $3 \mathrm{C}$ & $3 \mathrm{C}$ & N/A & $3 \mathrm{C}$ and possible $6 \mathrm{H}$ \\
\hline $\begin{array}{l}\text { Grain size of SiC crystals } \\
\text { - } \quad \text { TEM } \\
\text { - } \quad \text { EBSD }\end{array}$ & $\begin{array}{l}\text { Not included in this } \\
\text { report }\end{array}$ & Not included in this report & $\begin{array}{l}\text { Not included in } \\
\text { this report }\end{array}$ & $\begin{array}{l}\text { Not included in this } \\
\text { report }\end{array}$ \\
\hline $\begin{array}{l}\text { SiC inter-boundary layer } \\
\text { Properties: } \\
\text { - Thickness }\end{array}$ & $\begin{array}{l}\text { Average interlayer } \\
\text { thickness } 1.7 \mu \mathrm{m} \\
\text { (SEM montage) }\end{array}$ & $\begin{array}{l}\text { Average interlayer } \\
\text { thickness not determined } \\
\text { No debonding }\end{array}$ & N/A & $\begin{array}{l}\text { Not possible to } \\
\text { determine from } \\
\text { sample available }\end{array}$ \\
\hline - Debonding & No debonding & & & $\begin{array}{l}\text { No debonding } \\
\text { visible on limited } \\
\text { areas investigated }\end{array}$ \\
\hline Irradiation effects in $\mathrm{SiC}$ & Spherical cavities & $\begin{array}{l}\text { Angular cavities, may be } \\
\text { because of higher } \\
\text { temperature }\end{array}$ & N/A & $\begin{array}{l}\text { No cavities } \\
\text { observed. }\end{array}$ \\
\hline $\begin{array}{l}\text { Precipitates in } \mathrm{SiC} \text { or } \\
\mathrm{IPyC} / \mathrm{SiC} \text { interlayer } \\
\text { - Distribution } \\
\text { - Composition }\end{array}$ & Similar to $\mathrm{UPd}_{2} \mathrm{Si}_{2}$ & $\begin{array}{l}\text { Cs precipitates in } \\
\text { "wormlike" feature and in } \\
\text { grains. Wormlike feature is } \\
\text { both crosscutting } \\
\text { individual grains and } \\
\text { visible alongside grain } \\
\text { boundaries }\end{array}$ & N/A & $\begin{array}{l}\text { No } \mathrm{Pu} \text { in } \\
\text { precipitates } \\
\text { Similar to } \mathrm{UPd}_{2} \mathrm{Si}_{2}\end{array}$ \\
\hline & & Similar to $\mathrm{UPd}_{2} \mathrm{Si}_{2}$ & & \\
\hline $\mathrm{PyC}$ & $\mathrm{CP} 34$ & $\mathrm{CP} 35$ & CP39 & $\mathrm{CP} 30$ \\
\hline Irradiation effects in $\mathrm{PyC}$ & $\begin{array}{l}\text { Variations in SAD } \\
\text { rings }\end{array}$ & $\begin{array}{l}\text { More distinct variation in } \\
\text { SAD rings, may be because } \\
\text { of higher temperature }\end{array}$ & N/A & $\begin{array}{l}\text { Not possible to } \\
\text { determine from } \\
\text { sample available }\end{array}$ \\
\hline
\end{tabular}

\subsection{Comparison Between Low and High Ag-110m}

Two preliminary differences between the microstructure of the CP30 (high Ag-110m release) and those of CP34 and CP35 (relatively lower Ag-110m release) were observed, although any significance of these observations could not be determined at this stage because the statistical sample size is so small and the data needs to be validated with results obtained from future compacts. The two differences observed are as follows:

- No cavities because of irradiation in the SiC grains were observed during TEM examination of CP30, whereas cavities were observed in both the microstructure of the SiC grains of CP34 and CP35. This observation may suggest that CP30 was exposed to lower temperatures than CP34 and CP35.

- The EDS analysis of Pd-rich precipitates in CP30 indicated that they only contain very small $(\leq 1.3$ $w t \%)$ concentrations of $\mathrm{Pu}$ in contrast with those found in CP34 and CP35 which is in the order of 3 $\mathrm{wt} \%$. 


\section{RECOMMENDATIONS}

The TEM examination did not include full irradiation effects quantification and it is therefore recommended that this comparison with the $\mathrm{CP} 34$ and $\mathrm{CP} 35$ be done as a follow-up study. However, with this initial study, no obvious differences in $\mathrm{SiC}$ microstructures between these three samples were observed. It should be accentuated, however, that this work needs to be approached with a more detailed focus and specific objectives, which was not included in this preliminary study. These examinations need to be compared with the corresponding unirradiated material.

EPMA is a recommended WDS technique for determining both qualitative and quantitative concentrations of Ag, even at low concentrations and this technique will be used for analysis of irradiated particles from other compacts.

Recommendations include the verification of precipitate composition using TEM EDS with K $\alpha$ x-rays to differentiate between $\mathrm{Ag}, \mathrm{Pd}$, and $\mathrm{U}$. It is also recommended that a detailed precipitate analysis be done using EPMA and LEAP techniques, in conjunction with more in-depth investigation on the presence of fission products in intergranular and transgranular locations in the $\mathrm{SiC}$. It is also recommended that future work include the more detailed and quantitative study on cavities and dislocation loops.

Quantifying the PyC anisotropy on irradiated material is recommended because it may be significant to be able to use apparent anisotropy as an indicator of relative temperature. The role of neutron irradiation on the anisotropy of PyC needs also to be determined. 


\section{LESSONS LEARNED}

The following list summarizes the lessons learned from the electron microscopic examination of the first irradiated TRISO CPs of AGR-1 experiment:

- Various factors complicated the analysis of the TEM data, making it necessary to assume that all of the precipitates were similar. This assumption may not be true however, and will be evaluated in further studies.

- Many EDS spectra were not quantified, or were quantified based on incorrect elemental identifications.

- EDS spectra were typically saved as TIFF files showing the spectra themselves, and not in a format in which they could be reanalyzed.

- Either EDS data or diffraction patterns (but not both) were typically collected from an individual precipitate. Thus, it is generally not possible to definitively associate individual diffraction characteristics with a specific composition.

- In general, only one diffraction pattern was collected from each precipitate.

- The recording of the aperture size during SAD collection was introduced only in later stages of the TEM examination.

- $\quad \mathrm{Zr}$ can also be an artifact in EDX spectra collected with the objective aperture inserted.

- Quality (focus and resolution) of initial SEM micrographs was poor, resulting in rework.

- The basic SEM analysis was not always performed strictly per the established plan, resulting in some rework. Future work will be performed with close adherence to the plan to ensure all analyses are completed.

- Attempts to use TEM-EDS, SEM-EDS, and SEM-WDS to determine whether Ag was present using $\mathrm{X}$-rays in the $0-20 \mathrm{keV}$ range were unsuccessful because peak overlaps had not been properly identified in advance of data collection. 


\section{REFERENCES}

1 Petti, David, 2008a, "Project Execution Plan for the VHTR Technology Development Office," PLN-2494, Revision 0, Idaho National Laboratory (INL), April 18, 2008.

2 PLN 2826, “AGR-1 Post-Irradiation Examination,” Rev. 1, Idaho National Laboratory, March 2010.

3 Barrachin, M., Dubourg, R., de Groot, S., Kissane, M. P., Bakker, K., "Fission-product behavior in irradiated TRISO-coated particles: Results of the HFR-EUlbis experiment and their interpretation," Journal of Nuclear Materials, Vol. 415, 2011, pp. 104-116.

4 Kortright, J. B. and A. C. Thompson, "X-Ray Emission Energies," X-Ray Data Booklet 2009 [cited 2011 December 5, 2011]; Available from: http://xdb.lbl.gov/Section1/Sec_1-2.html.

5 Goldstein, J., et al., Scanning Electron Microscopy and X-Ray Microanalysis, Third Edition ed. 2003, New York, NY: Springer.

6 Shemirani, B., et al., "Magnetic structure of UPd2Si2," Physical Review B, 1993, Vol. 47, Issue 13.

7 Leciejewicz, J., H. Ptasiewicz-Bak, and A. Zygmunt, "Magnetic phase transitions in $\mathrm{UPd}_{2} \mathrm{Si}_{2,}, J$. Phys. Stat. Sol. (a), Vol. 51, 1979, pp. K71-K73.

8 Ptasiewicz-Bak, H., J. Leciejewicz, and A. Zygmunt, "Neutron diffraction study of magnetic ordering in $\mathrm{UPd}_{2} \mathrm{Si}_{2}, \mathrm{UPd}_{2} \mathrm{Ge}_{2}, \mathrm{URh}_{2} \mathrm{Si}_{2}$, and $\mathrm{URh}_{2} \mathrm{Ge}_{2}$, , J. Phys. F: Metal Phys., Vol. 11, 1981, pp. 1225-1235.

9 Marazza, R., et al., "Some phases in ternary alloys of thorium and uranium with the $\mathrm{Al}_{4} \mathrm{Ba}<\mathrm{ThCu}_{2} \mathrm{Si}_{2}$-type structure," Journal of the Less-Common Metals, Vol. 53, 1977, pp. 193-197.

10 Buschow, K. H. J. and D. B. De Mooij, "Structural and magnetic characteristics of several ternary compounds of the type $\mathrm{GdX}_{2} \mathrm{Si}_{2}$ and $\mathrm{UX}_{2} \mathrm{Si}_{2}(\mathrm{X}=3 \mathrm{~d}, 4 \mathrm{~d}$, or $5 \mathrm{~d}$ metal)," Philips Journal of Research, Vol. 41, 1986 pp. 55-76.

11 Brandes, E. A. and G. B. Brook, eds., Smithells Metals Reference Book, (Table 4.25), Seventh Edition, 1992, Butterworth-Heinemann, Ltd.: Oxford.

12 S. Kondo, Y. Katoh, L. L. Snead, "Microstructural defects in SiC neutron irradiated at very high temperatures," Journal of Nuclear Materials, Vol. 382, 2008, pp. 160-169.

13 Van Rooyen, I. J., J. Neethling, and J. Mahlanghu, "Influence of temperature on the micro-and nanostructures of experimental PBMR TRISO coated particles: A comparative study," HTR200858189, Proceedings of the 4th International Topical meeting on High Temperature Reactor Technology, HTR2008, September 28- October 1, 2008, Washington, DC USA.

14 Armour, K. J., PRD-350, "NGNP Quality Assurance program," rev 1, 2.0, Idaho National Laboratory, 03/04/2012.

15 LST-605, "Performer Controlled Activity Assessment for Operating the Focused ION Beam/Scanning Electron Microscope in the Electron Microscopy Laboratory," Idaho National Laboratory.

16 LST-514, "Skill of the Performer Assessment for Operating the JEOL JSM7000F Scanning Electron Microscope in the EML, Idaho National Laboratory.

17 LST-411, Skill of the Performer Assessment for Operating the JEOL 2010 Transmission Electron Microscope in the EML, Idaho National Laboratory. 